\title{
Lithium Pellet Injection Experiments on the Alcator C-Mod Tokamak
}

\author{
D.T. Garnier
}

May 1996

\begin{abstract}
DISCLAIMER
This report was prepared as an account of work sponsored by an agency of the United States Government. Neither the United States Government nor any agency thereof, nor any of their employees, makes any warranty, express or implied, or assumes any legal liability or responsibility for the accuracy, completeness, or usefulness of any information, apparatus, product, or process disclosed, or represents that its use would not infringe privately owned rights. Reference herein to any specific commercial product, process, or service by trade name, trademark, manufacturer, or otherwise does not necessarily constitute or imply its endorsement, recommendation, or favoring by the United States Government or any agency thereof. The views and opinions of authors expressed herein do not necessarily state or reflect those of the United States Government or any agency thereof.
\end{abstract}
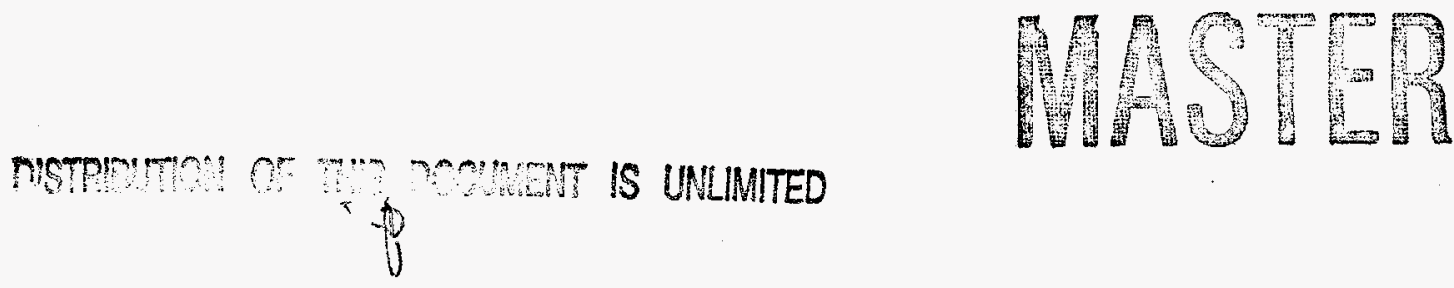

This work was supported by the U. S. Department of Energy Contract No. DE-AC0278ET51013. Reproduction, translation, publication, use and disposal, in whole or in part by or for the United States government is permitted. 


\section{DISCLAMMER}

Portions of this document may be illegible in electronic image products. Images are produced from the best available original document. 


\title{
Lithium Pellet Injection Experiments on the Alcator C-Mod Tokamak
}

\author{
by \\ Darren Thomas Garnier \\ A.B., Physics (1990) \\ University of California, Berkeley \\ Submitted to the Department of Physics \\ in partial fulfillment of the requirements for the degree of \\ Doctor of Philosophy in Physics \\ at the \\ MASSACHUSETTS INSTITUTE OF TECHNOLOGY \\ June 1996 \\ (C) 1996 Massachusetts Institute of Technology. All rights reserved.
}

Author

Department of Physics

May 17, 1996

Certified by

Earl S. Marmar

Senior Research Scientist

Thesis Supervisor

Accepted by

Prof. George Koster

Chairman, Departmental Committee on Graduate Students The Goverrment reserves for itself and others acting on its behalf a royalty free, nonexclusive, irrevocable, world-wide license for goverrmental purposes to publish, distribute, translate, duplicate, exhibit, and perform any such data opyrighted by the contractor. 


\title{
Lithium Pellet Injection Experiments \\ on the Alcator C-Mod Tokamak
}

by

\author{
Darren Thomas Garnier
}

\author{
Submitted to the Department of Physics \\ on May 17, 1996, in partial fulfillment of the \\ requirements for the degree of \\ Doctor of Philosophy in Physics
}

\begin{abstract}
A pellet enhanced performance (PEP) mode, showing significantly reduced core transport, is regularly obtained after the injection of deeply penetrating lithium pellets into Alcator C-Mod discharges. These transient modes, which typically persist about two energy confinement times, are characterized by a steep pressure gradient $\left(\ell_{p} \leq a / 5\right)$ in the inner third of the plasma, indicating the presence of an internal transport barrier. Inside this barrier, particle and energy diffusivities are greatly reduced, with ion thermal diffusivity dropping to near neo-classical values. Meanwhile, the global energy confinement time shows a $30 \%$ improvement over ITER89-P L-mode scaling. The addition of ICRF auxiliary heating shortly after the pellet injection leads to high fusion reactivity with neutron rates enhanced by an order of magnitude over L-mode discharges with similar input powers.

A diagnostic system for measuring equilibrium current density profiles of tokamak plasmas, employing high speed $(\sim 1 \mathrm{~km} / \mathrm{s})$ lithium pellets, is also presented. Because ions are confined to move along field lines, imaging the $\mathrm{Li}^{+}$emission from the toroidally extended pellet ablation cloud gives the direction of the magnetic field. To convert from temporal to radial measurements, the 3-D trajectory of the pellet is determined using a stereoscopic tracking system. These measurements, along with external magnetic measurements, are used to solve the Grad-Shafranov equation for the magnetic equilibrium of the plasma.

This diagnostic is used to determine the current density profile of PEP modes by injection of a second pellet during the period of good confinement. This measurement indicates that a region of reversed magnetic shear exists at the plasma core. This current density profile is consistent with TRANSP calculations for the bootstrap current created by the pressure gradient. MHD stability analysis indicates that these plasmas are near both the $n=\infty$ and the $n=1$ marginal stability limits.
\end{abstract}

Thesis Supervisor: Earl S. Marmar

Title: Senior Research Scientist 


\section{Acknowledgments}

I gratefully acknowledge the help of all the scientists, engineers, technicians and staff of the Alcator Group. They have aided me throughout this work; in the construction of the lithium pellet injector, the carrying out of the experiments on Alcator C-Mod and the subsequent data analysis. In particular, I must acknowledge: S. Wolfe for his help with the EFIT code, Y. Takase for aiding in the TRANSP analysis, J. Schachter for his assistance with the TRANSP code, and J. Ramos for his work on the ideal stabilty of PEP modes. P. Bonoli, M. Greenwald, J. Terry and others also have contributed many interesting and useful conversations. I would like to express my sincere thanks to my advisor, Earl Marmar, who has guided me throughout this work.

In addition, I would like to thank my readers M. Porkolab, P. Fisher and E. Marmar and my many other proofreaders and editors, for their helpful comments and suggestions.

My mental care and well-being was maintained by a cadre of friends and family; I am grateful for: My fellow graduate students, T. Hsu, C. Kurz, P. O'Shea, J. Reardon, J. Schachter, R. Nachtrieb, J. Urbahn and the rest who have made this doctoral journey more interesting and enjoyable. My parents, $\mathrm{Al}$ and JoLinda, who have given unending encouragement and support. My dog, Max, for his warm, enthusiastic and sometimes slobbery affection when I came home late from the lab. And most of all, for the love and support of my wife, Suanne, without whom I would not have completed this work. 
For Suanne 


\section{Contents}

1 Introduction $\quad 15$

2 Background $\quad 17$

2.1 Fusion and Magnetic Confinement . . . . . . . . . . . . . 17

2.1.1 Magnetic Confinement .............. 18

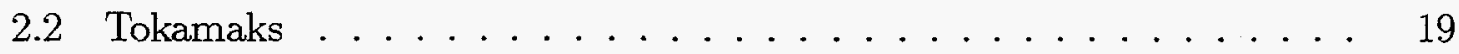

2.2.1 The Alcator C-Mod Tokamak . . . . . . . . . . . 22

2.3 MHD Equilibrium . . . . . . . . . . . . . . . 26

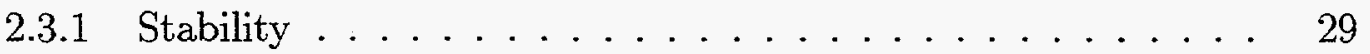

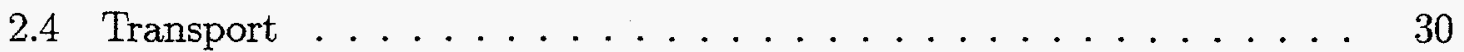

2.4.1 Classical Transport ................ 31

2.4.2 Neoclassical Transport ............... 31

2.4.3 Anomalous Transport. . . . . . . . . . . . 33

2.5 Equilibria and Transport . . . . . . . . . . . . . . . 34

3 Lithium Pellet ' $q$ ' Profile Diagnostic $\quad 37$

3.1 Equilibrium reconstruction . . . . . . . . . . . . . 38

3.2 Impurity Pellet Injector . . . . . . . . . . . . . . . . 41

3.2.1 Pellets: Size, Composition and Fabrication . . . . . . . . . 41

3.2 .2 Injector Design . . . . . . . . . . . . 43 
3.2.3 Performance.................... 50

3.3 Pellet Tracking System . . . . . . . . . . . . . . . 51

3.3.1 Stereoscopic Vision ................... 51

3.3 .2 Tracking Cameras ................. 52

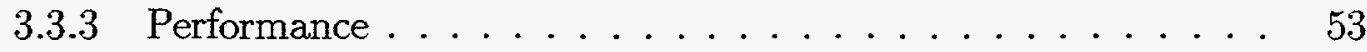

3.4 Ablation Cloud Imaging Diagnostic . . . . . . . . . . . . . . 55

3.4.1 Theoretical considerations .............. 56

3.4.2 Ablation Cloud Aspect Ratio . . . . . . . . . . . . . 61

3.4.3 The Cigar Detector . . . . . . . . . . . . 63

3.4.4 Cigar Detector Results . . . . . . . . . . . . . 65

3.4.5 Repetitive Shot Experiment ............. 67

4 Pellet Enhanced Performance $\quad 73$

4.1 Introduction . . . . . . . . . . . . . . . 74

4.2 Observed Characteristics of PEP mode . . . . . . . . . . . 76

4.2 .1 Shot $950609013 \ldots \ldots . \ldots \ldots$

4.2 .2 Peaked profiles .................... 82

4.2.3 Other PEP mode Experimental Results . . . . . . . . . . 85

4.3 PEP Mode Transport . . . . . . . . . . . . . . . . 86

4.3.1 Particle Confinement ............... 86

4.3.2 Thermal Transport Analysis .............. 91

4.4 PEP mode Equilibria . . . . . . . . . . . . . . . . 97

4.4.1 LPI $q$ profile measurements . . . . . . . . . . 97

4.4 .2 TRANSP $q$ profile modelling . . . . . . . . . . 100

4.4.3 Ideal MHD Stability Analysis . . . . . . . . . . . . . 104

4.5 Relationship with other enhanced confinement modes . . . . . . . . 111

4.5 .1 NCS/ERS mode ..................... 111 
4.5 .2 Other modes . . . . . . . . . . . . . . . . 113

4.6 Theoretical Implications and Speculation . . . . . . . . . . 114

$4.6 .1 \quad \eta_{i}$ Driven Modes . . . . . . . . . . . . . . . 115

4.6.2 Mechanisms for reduced transport . . . . . . . . 115

4.6 .3 Feedback and Causality . . . . . . . . . . 118

5 Conclusion

5.1 Summary . . . . . . . . . . . . . . . . . . . . 121

5.2 Further Investigations $\ldots \ldots \ldots \ldots \ldots \ldots \ldots$

Bibliography 


\section{List of Figures}

2.1 Schematic of a tokamak, indicating both cylindrical and toroidal coordinate conventions. . . . . . . . . . . . . . 20

2.2 Cross section of the Alcator C-Mod tokamak. . . . . . . . . . 24

2.3 Example Alcator C-Mod equilibrium. . . . . . . . . . . . . . 28

2.4 The banana shaped orbit of a trapped particle. . . . . . . . 32

2.5 The growth mechanism for the interchange mode. . . . . . . . . 34

2.6 Geometric effect of magnetic shear: two poloidal cross-sections of a density perturbation in a negative magnetic shear region for two different toroidal locations, separated by $\Delta \phi=\frac{\pi}{2} q \ldots \ldots \ldots \ldots \ldots$

2.7 Mechanism for magnetic shear stabilization of interchange modes. . . 36

3.1 Lithium pellet diagnostics used to measure magnetic field tilt angle on the Alcator C-Mod Tokamak. . . . . . . . . . . . . . 38

3.2 Arrangement of $B_{p}$ measurement coils on the vacuum vessel inside wall. 39

3.3 The Alcator C-Mod Impurity Pellet Injector. . . . . . . . . . . . . 43

3.4 Top view of the single-gas gun that accelerates the pellets to $\approx 1 \mathrm{~km} / \mathrm{s}$. 44

3.5 Front view of the gun showing the alignment of the barrels and the rings of holes in the pellet wheel magazine. . . . . . . . . . 46

3.6 The pellet wheel showing the Gray Code rotary encoding used to help keep an inventory database. . . . . . . . . . . . . . 47

3.7 Top view of injector showing the guide tubes, time-of-flight gates, and gas expansion free-flight gaps. 
3.8 Schematic diagram of injector vacuum and propellant gas systems. . .

3.9 Example trajectory of pellet through plasma as determined from stereoscopic tracking system. . . . . . . . . . . . . .

3.10 Gun sight view of pellet $\mathrm{Li}^{+}(5485 \AA)$ emission, time integrated over the entire pellet flight $(\approx 250 \mu \mathrm{s})$ using a standard CCD TV camera. The inner wall tiles are are made visible by edge plasma light during the $16 \mathrm{~ms}$ total exposure. . . . . . . . . . . . . .

3.11 Neutral gas shielding model of pellet ablation. . . . . . . . . 57

3.12250 ns snapshot of $\mathrm{Li}^{+}$ablation emission. Notice that ablation has filamenented into two distinct cigars. In the analysis, the upper filament, without the pellet and neutral cloud, is ignored. . . . . . . . . . .

3.13 Electron impact ionization rates for Lithium. . . . . . . . . . . . 62

3.14 Schematic of pellet ablation cloud imaging diagnostic used on TFTR.

3.15 Measured cigar tilt angles versus the magnetic field tilt angles calculated from the standard (not internally constrained) EFIT equilibrium reconstructions of many different discharges. . . . . . . . . .

3.16 Normal EFIT reconstruction for of plasma equilibrium during a sawtooth suppressed PEP mode. . . . . . . . . . . . . . . .

3.17 Comparison of cigar detector measurements $(\diamond)$ with tilts calculated $(\triangle)$ from EFIT reconstruction without internal constraints. . . . . . .

3.18 EFIT reconstruction using cigar detector data constraints gathered from a series of nominally identical shots. The data are taken during sawtooth suppressed PEP mode. . . . . . . . . . . . .

3.19 Comparison of cigar detector measurements $(\diamond)$ with tilts calculated $(\triangle)$ from EFIT reconstruction with constraints applied. . . . . . . .

3.20 EFIT reconstruction using cigar detector data constraints fitted with constraints but less internal freedom. A region of weakly reversed magnetic shear is suggested. . . . . . . . . . . . . .

3.21 Comparison of cigar detector measurements $(\diamond)$ with tilts calculated $(\triangle)$ from EFIT reconstruction with less internal freedom. . . . . . . .

4.1 Visible ablation light signal from pellet injected into plasma. . . . . . 
4.2 Expected electron density rise as a result result of pellet injection compared to pre- and post-pellet density measurements. (Shot 950609013.)

4.3 Time histories for an Alcator C-Mod discharge contrasting PEP and Lmode ICRF heated plasmas. Discharge conditions for shot 950426032: $B_{0}=5.3 \mathrm{~T}, I_{p}=1.0 \mathrm{MA}, \mathrm{H}$ minority ICRF heating scheme. . . . . .

4.4 Neutron rates for a number of different Alcator C-Mod discharges plotted versus total input power. The large symbols represent post-lithium pellet discharges that did not disrupt; not all of which exhibit the characteristics of an enhanced performance discharge. . . . . . . .

4.5 Time histories for Shot 950609013, an $8 \mathrm{~T} \mathrm{D-}{ }^{3} \mathrm{He}$ ICRF heated PEP mode. . . . . . . . . . . . . . . .

4.6 Plasma density and temperature profiles for shot 950609013 for three different times: Pre-pellet ohmic discharge $(760 \mathrm{~ms})$, PEP mode at the time of high reactivity $(830 \mathrm{~ms})$, and post-PEP L-mode $(920 \mathrm{~ms})$.

4.7 Total plasma pressure profiles for shot $950609013 \ldots \ldots \ldots$. . . . .

4.8 Surface plot of electron density profile as it evolves in time for shot 950609013. The stationary nature of the transport barrier is easily seen. 85

4.9 Time histories for $5.3 \mathrm{~T}, 800 \mathrm{kA}$ Ohmic PEP mode discharge, with lithium pellet injected at $0.8 \mathrm{~s} \ldots \ldots \ldots \ldots \ldots$

4.10 Time histories for two discharges with injection of Nickel at $0.79 \mathrm{sec}$. The dashed line signals are from a L-mode discharge. The solid shows a pellet injection at $0.80 \mathrm{sec}$ with a subsequent PEP mode. . . . . . .

4.11 Ion, electron and effective thermal diffusivities, as inferred by TRANSP, for three times during Shot 950609013. Shown for comparison is the calculated neoclassical ion diffusivity. . . . . . . . .

4.12 Radial power balance profiles for shot 950609013 at $830 \mathrm{~ms}$. . . . 96

4.13 Time histories from double pellet injection experiment used to measure the current density profile during PEP mode. The first pellet is injected at $0.6 \mathrm{~s}$ to produce the PEP mode, while the second pellet injected at 0.675 measures the current density profile. . . . . . . . . . 
4.14 Current density and $q$ profiles from EFIT equilibrium fit to 'Cigar Detector' measurements of the internal magnetic field tilt angle from repetitive shot PEP mode experiment. A hollow current profile and central region of weakly negative shear is clearly evident. . . . . . . .

4.15 Current density profiles from TRANSP poloidal field diffusion equilibrium modelling for Shot 950609013 during the PEP mode at $820 \mathrm{~ms}$, just before the peak of neutron rate. . . . . . . . . . . . . . . 101

4.16 Plasma parameters used or calculated by TRANSP to model the bootstrap current at $\rho=.2$. Also shown is $q_{0}$ and $q_{\min }$ as a function of time; when they deviate, a region of negative shear exists in the plasma core. 102

4.17 Current density and $q$ profiles from TRANSP modelling of shot 950609013 for $t=820 \mathrm{~ms}$. This equilibrium was used as a basis for the ideal MHD stability analysis. . . . . . . . . . . . . . 105

4.18 Ideal stability of shot 950609013 at $820 \mathrm{~ms}$ and discharges with similar equilibria. Equilibria that fall to the left or above the heavy lines are unstable to ideal modes. . . . . . . . . . . . . . . . . . . . . 108

4.19 Shown above is a contour plot of electron temperature radial profile versus time from the grating polychromator (GPC) ECE diagnostic near the termination of enhanced confinement for shot 950609013 . The actual temperature signals (lower plot) show clearly the loss of central enhanced confinement. 


\section{List of Tables}

2.1 Coordinate systems and naming conventions used in this document. . 21

2.2 Achieved Alcator C-Mod parameters. . . . . . . . . . . . . 23

2.3 Alcator C-Mod core diagnostics. . . . . . . . . . . . 25 


\section{Chapter 1}

\section{Introduction}

The next major step in the development of controlled nuclear fusion as a viable energy source is the construction of an "ignition machine". By definition, such a machine will be capable of obtaining the plasma parameters necessary for self-sustaining thermonuclear burning. The International Tokamak Experimental Reactor (ITER) is presently being designed to reach this goal. However, based on the scaling laws derived from the results of current experiments, the ITER design is an extremely large device and will be very expensive to build (given the current political climate in the US, perhaps prohibitively expensive). In the hopes that a less expensive means of reaching ignition might be found, a great deal of recent interest in the fusion community has focused on regimes of confinement that are enhanced compared to those from which the scaling laws are drawn. In this thesis we will examine the experiments conducted on the Alcator C-Mod tokamak exploring the enhanced regime of confinement known as Pellet Enhanced Performance (PEP) mode.

The structure of this thesis will adhere to the following outline. Chapter 2 briefly summarizes the field of magnetic confinement plasma fusion. The tokamak, magnetohydrodynamic (MHD) equilibrium and stability, and transport theory will be reviewed. Additionally, the relationship between enhanced confinement and magnetic 
equilibrium will be introduced.

In tokamaks, part of the magnetic field that confines the plasma is produced by current driven within the plasma. For this reason, the magnetic field within the plasma is not known a priori from the current in the external coils. On the Alcator C-Mod tokamak, a unique diagnostic system is employed to determine magnetic equilibrium of the plasma. The design and calibration of, and the results obtained from, the Lithium Pellet Injection ' $q$ ' profile diagnostic will be presented in Chapter 3.

In Chapter 4, we will explore the enhanced mode of confinement that is created after the injection of a large pellet into a tokamak plasma. Experimental measurements of plasma parameters documenting the improvement in confinement in PEP modes are presented along with numerical analysis of the transport coefficients. PEP mode plasma magnetic equilibria are experimentally determined as well as modelled from other experimental data. A MHD stability analysis of these discharges is presented. Comparison between PEP modes and other enhanced confinement regimes studied elsewhere is made and possible physical mechanisms for the enhancement in confinement are discussed.

In the concluding chapter, a summary of the results contained in this thesis is presented and further paths of inquiry are suggested based on these findings. 


\section{Chapter 2}

\section{Background}

\subsection{Fusion and Magnetic Confinement}

The experiments described in this dissertation were undertaken to further research which has as its ultimate goal the development of a controlled fusion energy reactor. Fusion, the combining of two light atoms to form a heavier one, produces a large amount of excess energy because the products of the reaction have less mass than the initial atoms. This missing mass is converted to energy.

Fusion as a source of energy has many potential advantages over current sources of energy. Unlike fossil fuels, it produces no airborne pollutants and creates no carbon dioxide or other greenhouse effect gases. Additionally, because the fuel for fusion is found in large quantities in seawater, fusion is a potentially inexhaustible source of energy. The initial candidates for fusion fuel are deuterium and tritium, both isotopes of hydrogen. Deuterium (D) is a stable isotope making up $0.015 \%$ of hydrogen found on earth. Tritium $(\mathrm{T})$ is not found in nature, but may be bred from lithium, a light element found in abundance in the earth's crust and oceans.

Unlike nuclear fission reactors, whose fuel supply for 1-2 years is present in the reactor, fusion reactors are not susceptible to the threat of meltdown. Radioactive 
waste from fusion power-plants will generally be limited to the decommissioned reactors themselves. And, through proper choice of building materials, the half-life of this radioactive waste can be reduced from the hundreds of thousands of years typical to nuclear waste from fission plants, to a few hundreds of years, thereby eliminating the very long term storage problems of spent nuclear fission fuel.

\subsubsection{Magnetic Confinement}

Fusion occurs at high temperatures where the reactant nuclei's kinetic energy can overcome the like-charge electrostatic repulsion and approach close enough for the nuclear strong force to dominate. Well before these temperatures are reached, atoms become ionized, freeing electrons from nuclei (ions). This ionized gas, composed of charged particles which can interact with each other over large distances and thus exhibit a large zoology of collective behavior, is called a plasma.

In order to reach the high temperatures needed for fusion, the plasma must be confined so that it does not lose energy and cool. A relatively simple relation, called the Lawson criterion, describes the condition necessary for thermonuclear burning of a D-T plasma;

$$
n_{i} \tau_{E} \gtrsim 1 \times 10^{20} \mathrm{~s} / \mathrm{m}^{3}
$$

for $T_{i} \sim 10 \mathrm{keV}$. Here $n_{i}$ is the ion density and $\tau_{E}$ is the energy confinement time. There are numerous mechanisms for plasma confinement, including gravitational, ${ }^{1}$ inertial, and electrostatic confinement. However, magnetic confinement is the most likely means of obtaining the parameters necessary for an earth-based controlled thermonuclear reactor.

\footnotetext{
${ }^{1}$ The sun is a thermonuclear fusion energy source whose plasma confinement is provided by a large gravitational field.
} 
The basis for magnetic confinement is the fact that charged particles follow helical paths around magnetic field lines as a result of the Lorentz force:

$$
\mathbf{F}=m_{j} \frac{d \mathbf{v}_{j}}{d t}=q_{j}\left(\mathbf{E}+\mathbf{v}_{j} \times \mathbf{B}\right)
$$

For the simplified case of no perpendicular electric field and uniform magnetic field, this yields the helical motion of particles along field lines with (Larmor) radius

$$
\rho_{L}=\frac{m v_{\perp}}{q B} .
$$

More generally, perpendicular electric fields, non-uniform magnetic fields or other forces perpendicular to the magnetic field lead to perpendicular drifts of the particle orbital centers (guiding centers), with drift velocity given by

$$
\mathbf{v}_{D}=\frac{1}{q} \frac{\mathbf{F}_{g} \times \mathbf{B}}{B^{2}}
$$

where $\mathbf{F}_{g}$ is any generalized force. For a complete introduction to single particle orbits and basic plasma physics, the interested reader is referred to the texts by Chen [1] and Nicholson [2]. A more thorough treatment of magnetic confinement of plasmas is given in Hazeltine and Meiss [3].

\subsection{Tokamaks}

Although numerous other configurations have been tried over the nearly 50 year course of magnetic confinement fusion research, the most successful and consequently best studied device is the tokamak. The tokamak is a closed field device; the magnetic field lines, along which plasma particles travel, are looped back on themselves and are completely contained within the device. Topologically, the tokamak is a torus. A 


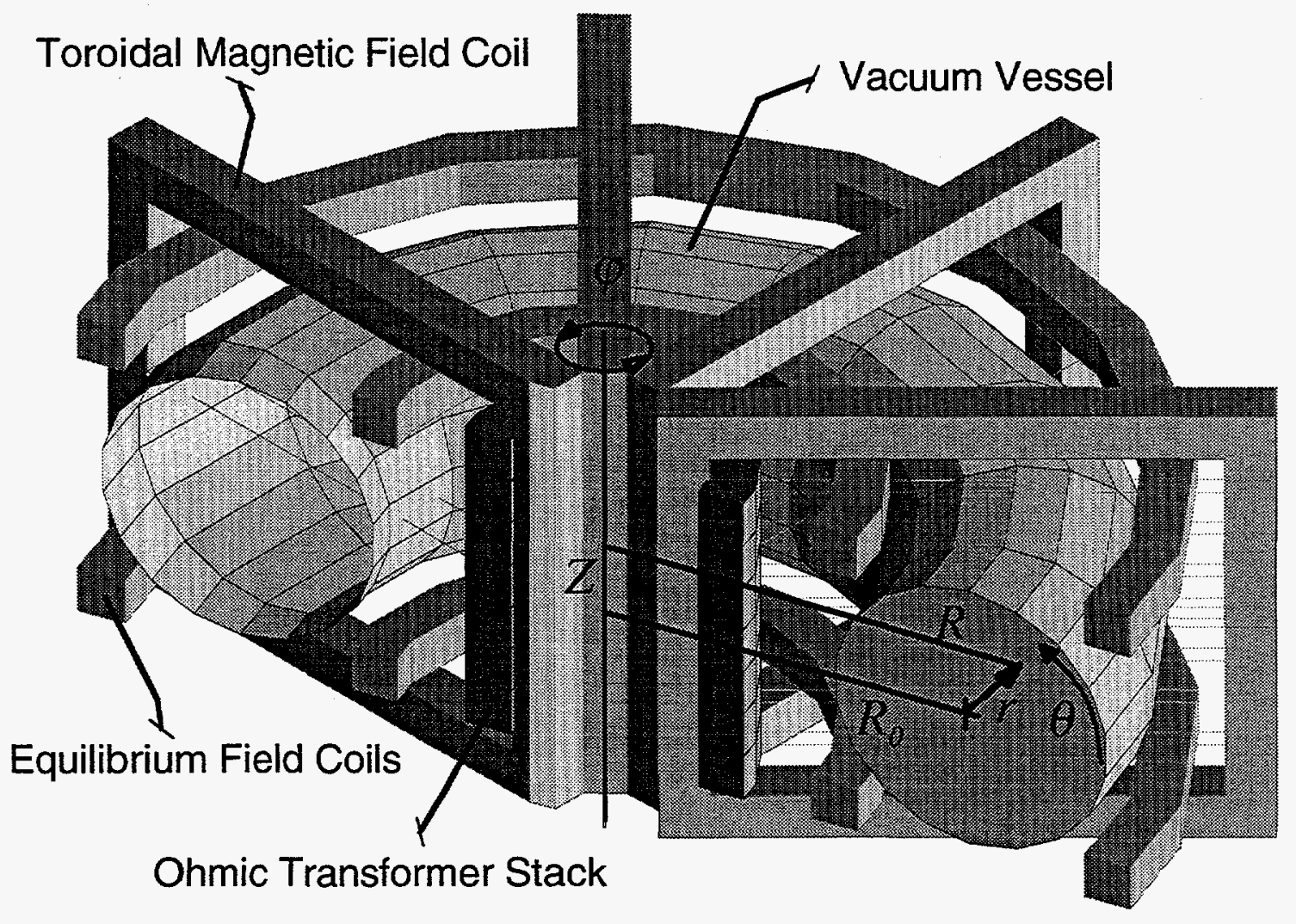

Figure 2.1: Schematic of a tokamak, indicating both cylindrical and toroidal coordinate conventions.

schematic of the tokamak, along with the various geometric conventions used in the fusion community are shown in Fig. 2.1. The coordinate systems employed in this document are summarized in Table 2.1.

When a magnetic field is formed into the shape of a torus, it is inherently curved and non-uniform. As mentioned in the previous section, this leads to plasma particle drifts, the so-called $\nabla \mathrm{B}$ and curvature drifts:

$$
\mathbf{v}_{\nabla \mathbf{B}, \mathbf{R}_{c}}=\frac{T_{j}}{q_{j}} \frac{\mathbf{B} \times \nabla \mathbf{B}}{B^{2}}
$$

For a toroidal field, this drift is in the vertical direction, and because this drift is 


\section{Tokamak Coordinate Systems}

\begin{tabular}{|l|l|l|l|}
\hline Cylindrical & $R$ & $Z$ & $\phi$ \\
\hline Toroidal & $r$ & $\theta$ & $\phi$ \\
\hline Flux & $\psi$ & $\vartheta$ & $\phi$ \\
\hline & $=$ toroidal direction $\theta=$ poloidal direction \\
$R$ & $=$ major radius $\quad R_{0}=$ major radius of plasma axis \\
$r$ & $=$ minor radius $\quad a=$ minor radius of edge of plasma
\end{tabular}

Table 2.1: Coordinate systems and naming conventions used in this document. Flux coordinates will be described in $\S 2.3$

sensitive to the sign of the charge of the drifting particle, electrons and ions will drift in opposite directions. For a purely toroidal field, this would lead to charge separation and the creation of a vertical electric field. Such a field would cause an outward $\mathbf{E} \times \mathbf{B}$ drift of both ions and electrons.

To overcome particle drifts, toroidal confinement devices are given a helical magnetic field, which averages out the effect of these drifts. Suppose a particle drifts upward while travelling along a field line. When at the highest point of the field line, the particle drifts outward in minor radius; however, when at the bottom, the particle drift is inward, towards the center of the plasma. Thus, on average, the particle drift is zero. (However, because of the particle drifts, the guiding center motion is not exactly along the field line; this fact will be important later in §2.4.2.)

The helical field in a tokamak can be broken into two components: the toroidal field, $B_{\phi}$ (sometimes written $B_{T}$ ), and the poloidal field, $B_{\vartheta}$ (or $B_{P}$ ). $B_{\phi}$ is created by the external toroidal field (TF) coils and is inversely proportional to major radius $\left(B_{\phi} \propto 1 / R\right.$.) What distinguishes a tokamak from other toroidal confinement devices is that the poloidal field in a tokamak is created by toroidal current carried by the plasma itself.

The plasma current is driven inductively by ramping the current in a vertical solenoid magnet at the center of the tokamak called the ohmic $(\mathrm{OH})$ coil. Thus, the 
tokamak resembles an electrical transformer with the $\mathrm{OH}$ coil as the primary and the plasma as the secondary coil. The plasma current also heats the plasma through

resistive (or ohmic) heating $\left(P_{O H}=\eta j^{2}\right)$, hence the name ohmic coil. Tokamaks are typically also heated by other auxiliary means, including high energy neutral beam injection (NBI), ion cyclotron resonant radio frequency (ICRF) heating or electron cyclotron resonant microwave heating (ECH). Plasma current can also be driven using ICRF or NBI.

A tokamak has other coils that wrap toroidally around the device. Generically called equilibrium field (EF) coils, they that are used to maintain the plasma position and shape.

One should note that tokamaks are axisymmetric. Except for a slight variation in the toroidal field caused by the finite spacing of the TF coils, there is no intrinsic dependence on the toroidal coordinate, $\phi$. This simplifies much of the theoretical and experimental research conducted on tokamaks.

\subsubsection{The Alcator C-Mod Tokamak}

The research presented in this thesis was conducted on the Alcator C-Mod tokamak. Alcator C-Mod is a compact, high magnetic field, high density, shaped, diverted tokamak. Although Alcator C-Mod is linearly one third to one half as large as other current fusion research devices, the high particle and energy densities achieved make it relevant to the development of ignited plasmas. A list of some of the important parameters of Alcator C-Mod are shown in Table 2.2. A cross section of Alcator C-Mod is shown in Fig. 2.2 depicting the overall structure of the machine. 


\begin{tabular}{|l|c|cl|}
\hline Major Radius & $R_{0}$ & $0.67^{a}$ & $\mathrm{~m}$ \\
\hline Minor Radius & $a$ & $0.21^{a}$ & $\mathrm{~m}$ \\
\hline Magnetic Field & $B_{T}$ & $7.9(9)^{b}$ & $\mathrm{~T}$ \\
\hline Plasma Current & $I_{p}$ & $1.2(3)^{b}$ & $\mathrm{MA}$ \\
\hline Auxiliary Heating & $P_{I C R F}$ & $3.5(8)^{c}$ & $\mathrm{MW}$ \\
\hline Elongation & $\kappa$ & $0.95-1.85$ & \\
\hline Triangularity & $\delta$ & $0 .-0.4$ & \\
\hline Electron Density & $n_{e}$ & $\leqslant 1.1 \times 10^{21}$ & $\mathrm{~m}^{-3}$ \\
\hline Electron Temperature & $T_{\epsilon}$ & $\leqslant 6$ & $\mathrm{keV}$ \\
\hline Current Flattop Time & & $\sim 1$ & $\mathrm{~s}$ \\
\hline
\end{tabular}

${ }^{a}$ nominal. ${ }^{b}$ design maximum. ${ }^{c}$ planned.

Table 2.2: Achieved Alcator C-Mod parameters. 


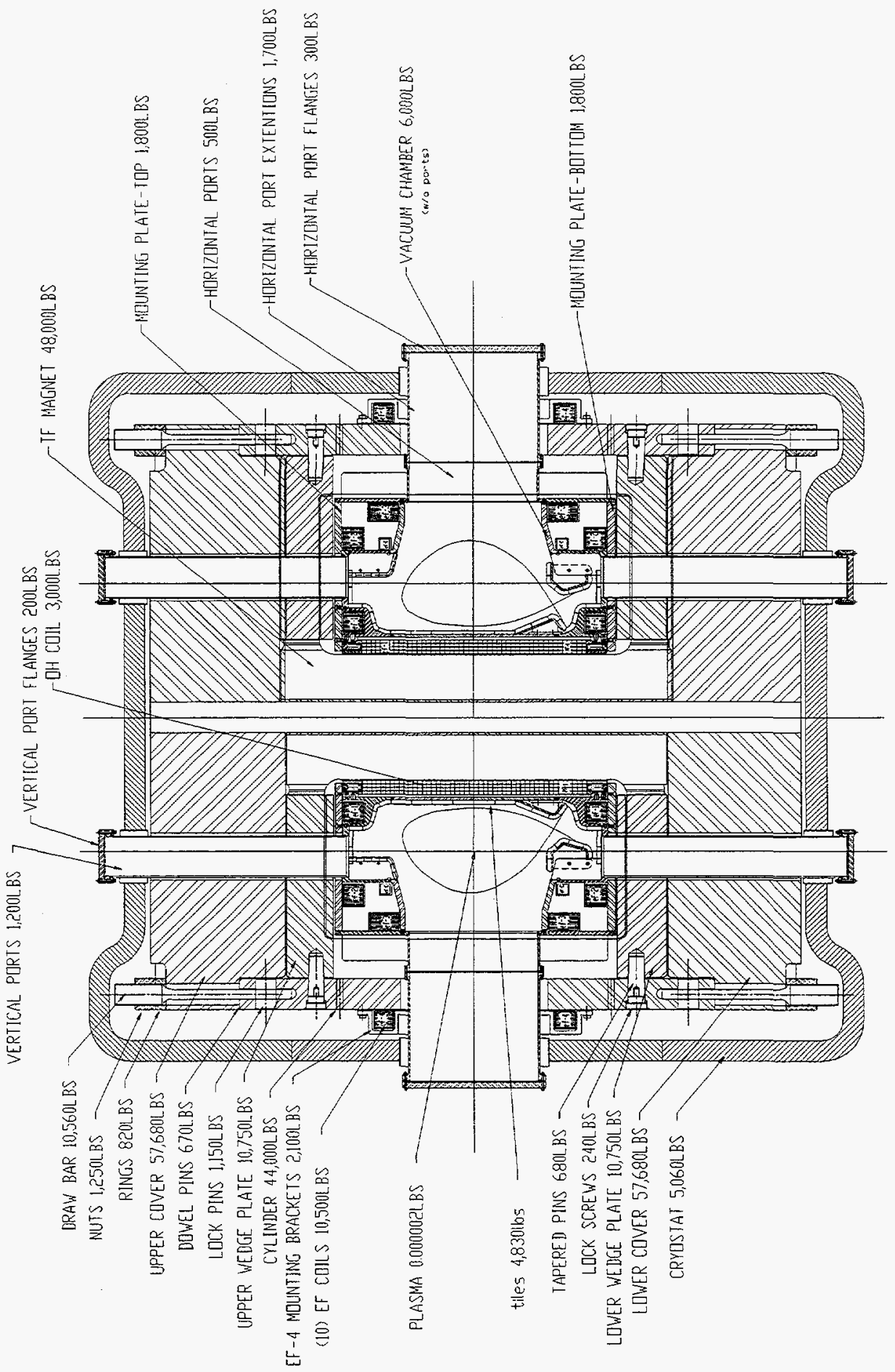

Figure 2.2: Cross section of the Alcator C-Mod tokamak. 


\begin{tabular}{|l|c|l|}
\hline Diagnostic & Measures & Comments \\
\hline \hline $\begin{array}{l}\text { Electron Cyclotron Emission } \\
\text { (ECE) diagnostics }\end{array}$ & $T_{e}$ & $\begin{array}{l}\text { Two distinct diagnostics give good } \\
\text { spacial and temporal resolution }\end{array}$ \\
\hline Two-color interferometry & $\int n_{e} d l$ & $\begin{array}{l}\text { Vertical chordal density mea- } \\
\text { surement, reconstruction of } n_{e} \text { is } \\
\text { unreliable. }\end{array}$ \\
\hline Thomson Scattering & $n_{e}, T_{e}$ & $\begin{array}{l}\text { Local measurement but incomplete } \\
\text { temporal and spatial resolution }\end{array}$ \\
\hline Reflectometer & $n_{e}, \tilde{n}_{e}$ & $\begin{array}{l}\text { Good for low density discharges, can } \\
\text { also measure fluctuations. }\end{array}$ \\
\hline Bolometer & $P_{r a d}$ & Radiated power measurement \\
\hline Neutron detectors & $R_{D D}, T_{i}$ & $\begin{array}{l}\text { Determines fusion rate. } T_{i} \text { can be de- } \\
\text { termined by modelling. }\end{array}$ \\
\hline HIREX & $T_{i}, v_{i}$ & $\begin{array}{l}\text { High resolution x-ray spectroscopy. } \\
\text { Doppler shift measurement of } v_{i}> \\
1 \times 10^{4} \mathrm{~m} / \mathrm{s} .\end{array}$ \\
\hline Visible Bremsstrahlung & $Z_{e f f}$ & $\begin{array}{l}\text { Requires good density data to be } \\
\text { accurate. }\end{array}$ \\
\hline Soft X-ray arrays & $\begin{array}{l}142 \text { channels for tomographic recon- } \\
\text { struction of emissivity in poloidal } \\
\text { cross section. }\end{array}$ \\
\hline
\end{tabular}

Table 2.3: Alcator C-Mod core diagnostics.

\section{Alcator C-Mod Diagnostics}

On Alcator C-Mod numerous instruments are used to diagnose the condition of the plasma. Although a complete description of the Alcator diagnostic set goes beyond the scope of this introduction, a list of the core (as apposed to edge) diagnostics is given in Table 2.3. For more information on the diagnostic set, the curious reader is referred to Marmar et al [4]. For a thorough introduction to plasma diagnostics in general, see Hutchinson [5]. 


\subsection{MHD Equilibrium}

Ideal magnetohydrodynamics (MHD) is a simple, but extremely useful, description of a plasma. By treating the plasma as a perfectly conducting single fluid one obtains a closed set of equations that describe the plasma state. In steady-state these equations reduce to [6]:

$$
\begin{aligned}
\mathbf{j} \times \mathbf{B} & =\nabla p \\
\mathbf{E}+\mathbf{v} \times \mathbf{B} & =0 \\
\nabla \times \mathbf{B} & =\mu_{0} \mathbf{j} \\
\nabla \times \mathbf{E} & =0 \\
\nabla \cdot \mathbf{B} & =0 .
\end{aligned}
$$

Here $\mathbf{E}$ and $\mathbf{B}$ are the electric and magnetic fields, $p$ is the plasma pressure, $\mathbf{v}$ is the plasma flow velocity and $j$ is the plasma current. If we allow finite plasma resistivity, Eq. (2.7) becomes

$$
\mathbf{E}+\mathbf{v} \times \mathbf{B}=\eta \mathbf{j}
$$

the modified Ohm's law. This addition defines "resistive" MHD. Many texts give derivations of the MHD equations [1,2], with a thorough treatment given by Freidberg [6]. Only a brief review of equations relevant to this thesis is given here.

We seek a description of the overall configuration of the plasma in steady state. The Grad-Shafranov equation describes all axisymmetric (cylindrical geometry) ideal MHD equilibria:

$$
\Delta^{*} \psi=-F \frac{\partial F}{\partial \psi}-\mu_{0} R^{2} \frac{\partial p}{\partial \psi}
$$


Here $p$ is the plasma pressure and $F$ is related to toroidal magnetic field by

$$
F=R B_{\phi}
$$

$\psi$ is the poloidal magnetic flux and is related to the poloidal field $\mathbf{B}_{\vartheta}$ by

$$
\mathbf{B}_{\vartheta}=\frac{1}{R} \nabla \psi \times \mathbf{e}_{\phi}
$$

The left hand side of the Grad-Shafranov equation is related to the toroidal current density, $j_{\phi}$ :

$$
\begin{aligned}
\Delta^{*} \psi & =R^{2} \nabla \cdot\left(\frac{\nabla \psi}{R^{2}}\right) \\
& =-\mu_{0} R j_{\phi}
\end{aligned}
$$

An example solution to the Grad-Shafranov equation is depicted in Fig. 2.3

The poloidal flux, $\psi$, is an important parameter in plasma equilibria. Notice that

$$
\nabla \psi \cdot \mathbf{B}=\nabla \psi \cdot\left(\mathbf{B}_{\vartheta}+B_{\phi} \mathbf{e}_{\phi}\right)=0
$$

indicating that $\nabla \psi \perp \mathbf{B}$. Therefore, magnetic field lines are drawn on surfaces of constant flux. Furthermore, inspection of the Grad-Shafranov equation reveals that $F$ and $p$ are functions of $\psi$. This makes $\psi$ a useful generalized radial coordinate, and so-called "flux coordinates" are often used in describing plasma properties that are inherently one-dimensional, depending only on $\psi$. (Although one-dimensional quantities are typically computed as functions of $\psi$ they are often displayed versus radius on the outside mid-plane of the tokamak. It is also customary to use cylindrical coordinates $(R, Z)$ instead of the flux coordinates $(\psi, \vartheta)$ when describing two dimensional data.) 

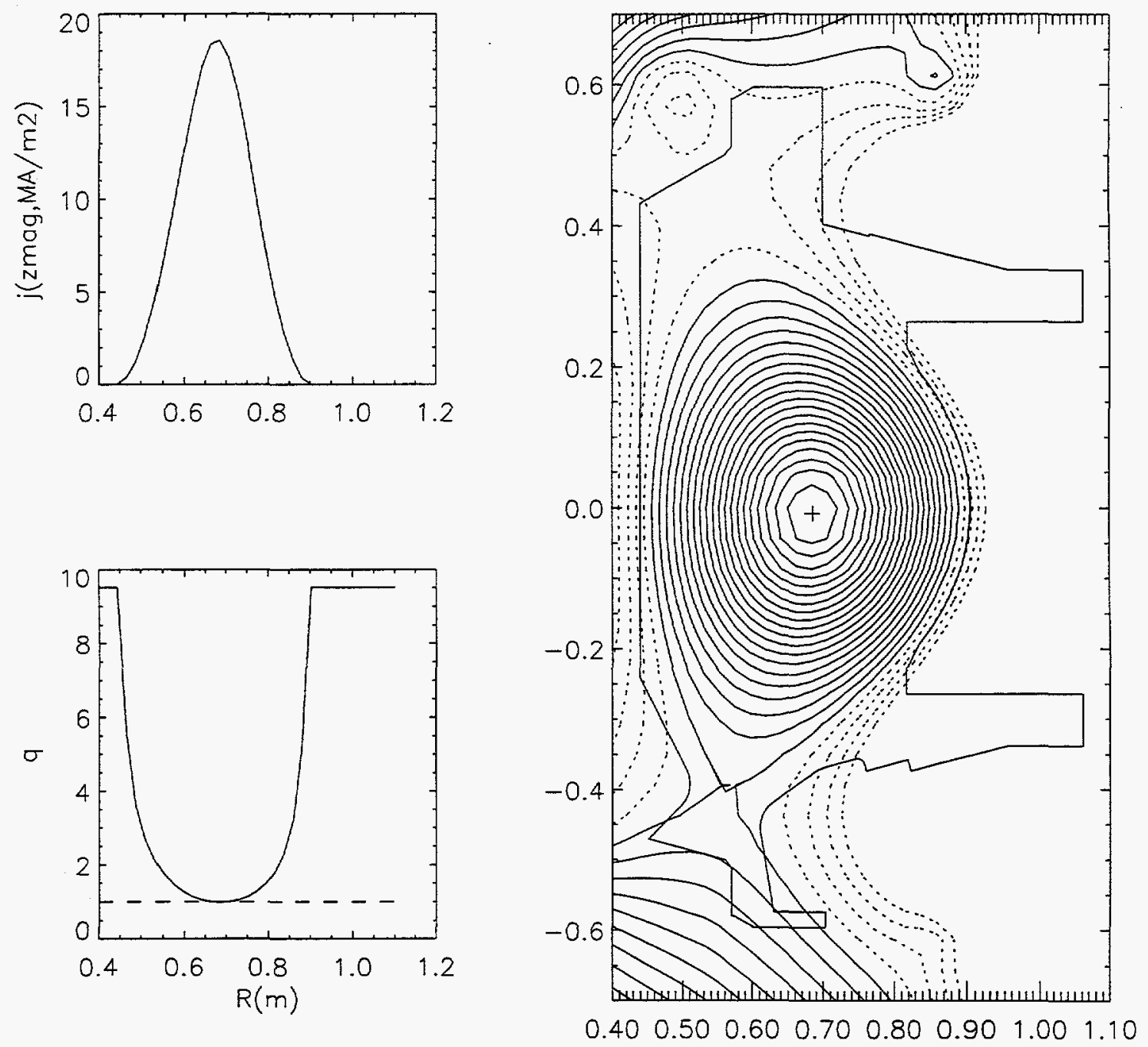

Figure 2.3: Contours of constant poloidal flux indicate the shape of the of a typical Alcator C-Mod plasma equilibrium. The last solid contour is the separatrix. On the left, the current density, $j$, and safety factor, $q$ radial profiles at the plasma midplane are shown. (Output from the EFIT code, which numerically solves the Grad-Shafranov equation. EFIT will be discussed in §3.1.) 
Another important parameter describing the equilibrium of the plasma is the socalled magnetic "safety factor", $q$. The safety factor is a measure of the helicity of the magnetic field lines on a flux surface and is defined as the number of toroidal circuits a field line makes for every poloidal circuit over the flux surface. Therefore (in toroidal coordinates),

$$
q(\psi)=\frac{1}{2 \pi} \int_{0}^{2 \pi}\left(\frac{r B_{\phi}}{R B_{\theta}}\right)_{S} d \theta
$$

where $S$ indicates that the integral is to be computed on the flux surface. The more easily computed local safety factor, $q_{l}$ is given by

$$
q_{l}=\frac{r B_{\phi}}{R B_{\theta}}
$$

Notice, however, that $q_{l}$ is generally not a flux function $\left(q_{l} \neq q_{l}(\psi)\right)$. Because the poloidal field is a result of the plasma current, $q$ generally relates the experimentally controllable magnetic field and plasma current. A global variation of the safety factor is $q^{*}$ :

$$
q^{*}=\frac{2 \pi \kappa a^{2} B_{0}}{\mu_{0} I_{p} R_{0}}
$$

where $B_{0}$ is the value of the vacuum toroidal magnetic field at the plasma axis, $R_{0}$, and $I_{p}$ is the total plasma current.

\subsubsection{Stability}

The reason that $q$ is called the "safety factor" is that it is an important parameter regarding the stability of a plasma equilibrium. As not all equilibria are minimum energy states, instabilities arise driven by plasma current (kink instabilities) or pressure 
(ballooning instabilities).

For example, the sawtooth oscillation [7], named for saw-like shape of the plasma parameter time histories, and clearly visible on the core electron temperature and soft $\mathrm{x}$-ray emissivity signals, is present in most tokamak discharges. At a sawtooth crash, energy (and sometimes density) is rapidly transported out of the core region to the surrounding plasma, flattening the temperature profile. Outside the core region the temperature is increased at a sawtooth crash, inverting the saw-like time histories.

The sawtooth oscillation is generally believed to be associated with the $m=1, n=$ 1 internal kink instability which becomes unstable in ideal MHD stability analysis for $q<1$. The sawtooth inversion radius is taken to be at or near the $q=1$ surface where the mode is resonant. Thus changes in the behavior of sawteeth generally indicate a change in the $q$ profile.

\subsection{Transport}

The confinement of a plasma, even in equilibrium, is not perfect. Particle collisions and plasma collective effects cause particles and energy to move across field lines and become lost from the plasma. This motion is called transport. In this section, we will review transport theory by looking at the equations governing particle transport. (Momentum and energy transport can be treated similarly although the equations are slightly more complicated.)

We begin with the equation describing the conservation of particles:

$$
\frac{\partial n}{\partial t}+\nabla \cdot \Gamma=S
$$

where $n$ is the particle density and $S$ is the particle source or sink (generally $S$ can be ignored except near the edge of the discharge where a significant neutral density 
exists). The flux of particles through a given surface, $\Gamma$, is given by

$$
\Gamma=-D \nabla n+n v
$$

where the transport coefficients, $D$ and $v$, are, respectively, the particle diffusivity and the convective velocity (sometimes called the "pinch" velocity when directed inward).

\subsubsection{Classical Transport}

The minimum level of transport is the diffusivity caused by particle collisions. In general the diffusivity due to a random walk process is given by [3]

$$
D_{\text {random walk }}=(\text { step-size })^{2} \times(\text { collision frequency }),
$$

where the step-size is the excursion between collisions. For magnetically confined particles, the maximum cross-field excursion between collisions is the Larmor radius. Thus

$$
D_{c}=\rho_{L}^{2} \nu_{c},
$$

where $\nu_{c}$ is the collision frequency and is calculated from the cross-section electrostatic scattering through $90^{\circ} . D_{c}$ is known as the "classical" diffusivity, and is relevant to plasmas in linear, homogeneous magnetic fields.

\subsubsection{Neoclassical Transport}

"Neoclassical" transport theory properly treats complications arising in toroidal confinement devices [8]. Some effects are purely geometric; e.g. helicity causes transport coefficients to gain a term proportional to $\left(1+q^{2}\right)$. A fully kinetic treatment is neces- 
sary because the population of particles on a flux surface is slightly non-maxwellian. Because $|\mathbf{B}|$ varies along a field line, particles with large orbital pitch, $v_{\perp} / v_{\|}$are reflected by the high magnetic field on the inside of the tokamak. Thus, some fraction of the plasma is trapped on the outboard side of the discharge. Because these trapped particles still undergo the $\nabla B$ drift, the overall path of a trapped particle resembles a banana (see Fig. 2.4). The untrapped particles are typically referred to as "passing" particles.

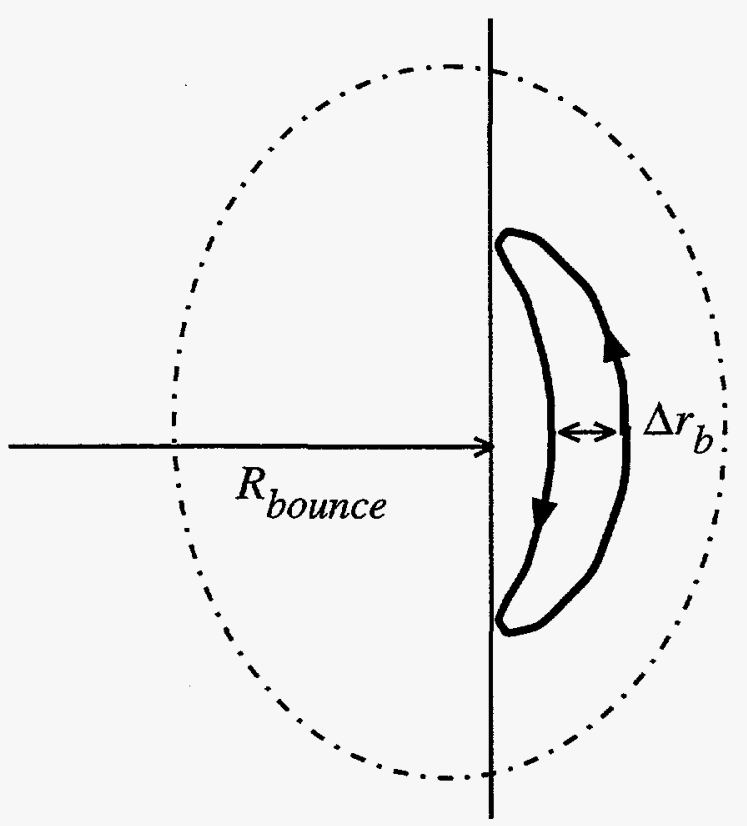

Figure 2.4: The "banana"-shaped poloidal projection of the guidingcenter motion of a trapped particle. The width of the orbit is known as the "banana width", $\Delta r_{b}$.

The maximum possible radial excursion between collisions is given by the width of this banana orbit, $\Delta r_{b}$. Thus, our heuristic argument suggests

$$
D_{\text {neo }} \sim \Delta r_{b}^{2} \nu_{c} .
$$

As $\Delta r_{b} \gg \rho_{L}$, this implies, correctly, that the neoclassical transport is much bigger 
than classical transport. (This functional dependence for the neoclassical diffusivity is only valid in the low collisionality "banana" regime. This regime is defined for $\nu_{c} \ll \omega_{b}$, where $\omega_{b}$ the trapped particle bounce frequency.)

Neoclassical transport theory also predicts some effects that do not exist in the classical treatment. For example, the toroidal electric field, $E_{\phi}$, drives an inward convection of particles (the Ware [9] pinch.) Also, friction between trapped and passing electrons in the presence of a density gradient can drive toroidal current-the so-called "bootstrap" current, $j_{B S}$. For more information on neoclassical transport, see the review article by Hinton and Hazeltine [8] or the text by Hazeltine and Meiss [3]

\subsubsection{Anomalous Transport}

Unfortunately, the level of transport measured in tokamaks is typically much larger than that predicted by the neoclassical theory. This "anomalous" transport can only be explained by the collective behavior of plasmas. Unstable plasma waves, or "modes" in the plasma create turbulence that scatter plasma particles causing transport that can be orders of magnitude larger than neoclassical levels. (The theoretical framework for describing how collective modes affect plasma transport is given by Braginskii [10].) A full understanding of the modes that drive anomalous transport remains a key goal of plasma fusion research. 


\subsection{Equilibria and Transport}

The plasma pressure and current density profiles in equilibrium can significantly influence the growth rates of the plasma waves that causing anomalous transport. In this section we will examine how a particular type of plasma wave, called the "interchange mode" [1] is affected by the equilibrium $q$ profile.
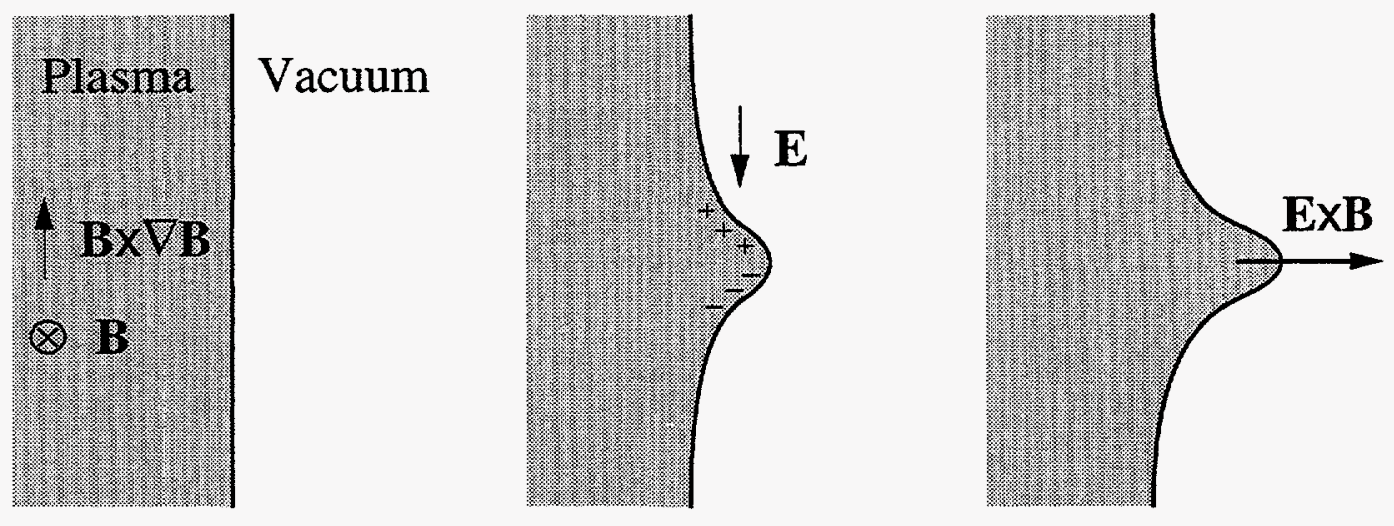

Figure 2.5: The growth mechanism for the interchange mode.

A simple model illustrating the driving mechanism of interchange modes is shown in Fig. 2.5. In this model, a distinct boundary exists between a volume of plasma and the vacuum that surrounds it. Parallel with this boundary is a charge dependent particle drift (for example, the $\nabla B$ drift.) If a perturbation in this boundary is introduced, the drifting ions and electrons now lead to charge separation in the perturbation. This polarization gives a vertical electric field which in turn causes an outward $\mathbf{E} \times \mathbf{B}$ drift. Thus, the amplitude of the perturbation is increased and the mode grows.

Next, we apply this model to tokamak geometry. Recall that the safety factor, $q$, is a measure of the helicity of a flux surface. Since the value of $q$ varies across the plasma profile, the field lines of adjoining flux surfaces are sheared with respect to one another. Formally, the magnetic shear, $s$, is the radial derivative of $q$ and defined 
by [6]

$$
s=2 \frac{V}{q} \frac{d q}{d V}
$$

where $V(\psi)$ is the volume contained in each flux surface. As tokamaks typically have positive shear everywhere in the discharge, negative shear is often referred to as "reversed shear".

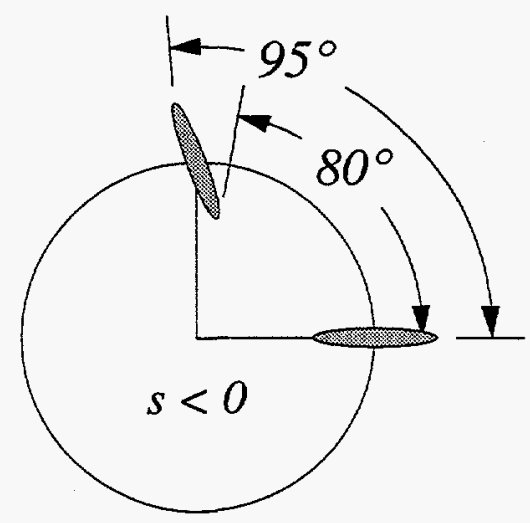

Figure 2.6: Geometric effect of magnetic shear: two poloidal crosssections of a density perturbation in a negative magnetic shear region for two different toroidal locations, separated by $\Delta \phi=\frac{\pi}{2} q$.

Magnetic shear will rotate a density perturbation with finite radial extent with respect to its minor radius direction. The reversed shear causes the poloidal rotation of the magnetic field to be faster on the outer flux surfaces than on the inner surfaces. As shown in Fig. 2.6, this tilts the perturbation inward at the top of the flux surface.

Returning our attention to the interchange mode, shown now for toroidal geometry in Fig. 2.7, we see that tilt has the effect of stabilizing the mode. Shown are the three cases of normal, zero, and negative magnetic shear. Since the $\nabla B$ drift is in the vertical direction everywhere in the discharge, the polarization and $\mathbf{E} \times \mathbf{B}$ drift of density in the perturbation will remain the same on top of the flux surface. However, 

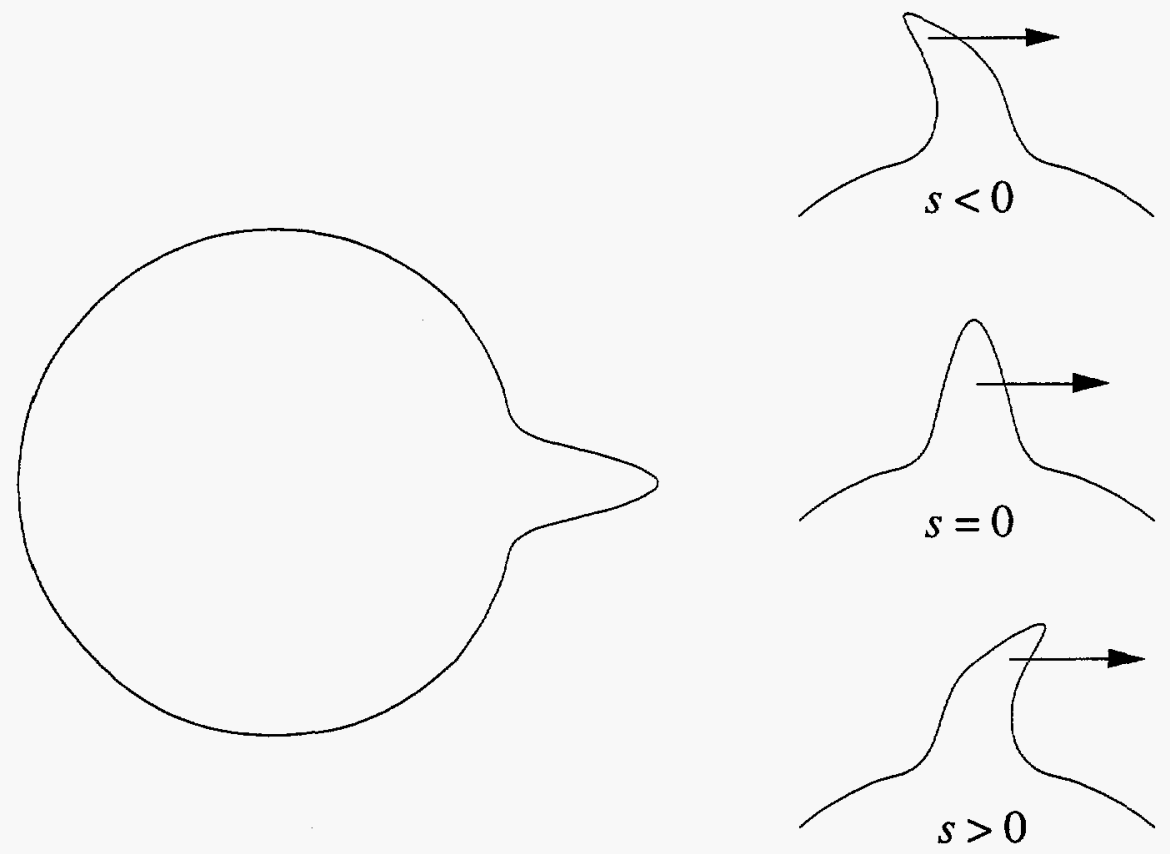

Figure 2.7: Mechanism for magnetic shear stabilization of interchange modes.

the drift outward in major radius will have a different effect on each of the different shear cases. For normal shear, the effect is to grow the perturbation, while for the negative magnetic shear it has a stabilizing effect. (The zero shear case is marginally stable.)

This effect of the magnetic shear on the growth rate of plasma modes is formally known as "favorable geodesic curvature." The magnetic shear affects tokamak discharges in other ways, as well. For example, high magnetic shear is generally good for ideal MHD stability, while a secondary region of stability exists for high $\beta$ and low or negative shear. For these reasons, determination of the $q$ profile is an important diagnostic for understanding tokamak discharges. 


\section{Chapter 3}

\section{Lithium Pellet ' $q$ ' Profile Diagnostic}

Measurement of the current density profile on Alcator C-Mod is accomplished using a lithium pellet as an internal probe of the magnetic field. The current density diagnostic system is composed of five separate parts. First, there is the impurity pellet injector, which is capable of injecting high speed lithium pellets through the core of the plasma. Second, there is the pellet tracking system that measures the trajectory of the pellet. Next are the two independent diagnostics that return the magnetic field tilt angle at the position of the pellet: the ablation cloud imaging diagnostic or 'Cigar Detector' and the Zeeman polarimetry system. The arrangement of the pellet diagnostics on the tokamak is shown in Fig. 3.1. Finally, a least-squares fitting code (EFIT) is used to solve for the magnetohydrodynamic equilibrium of the plasma fitted to the pellet measurements and other external magnetic measurements. 


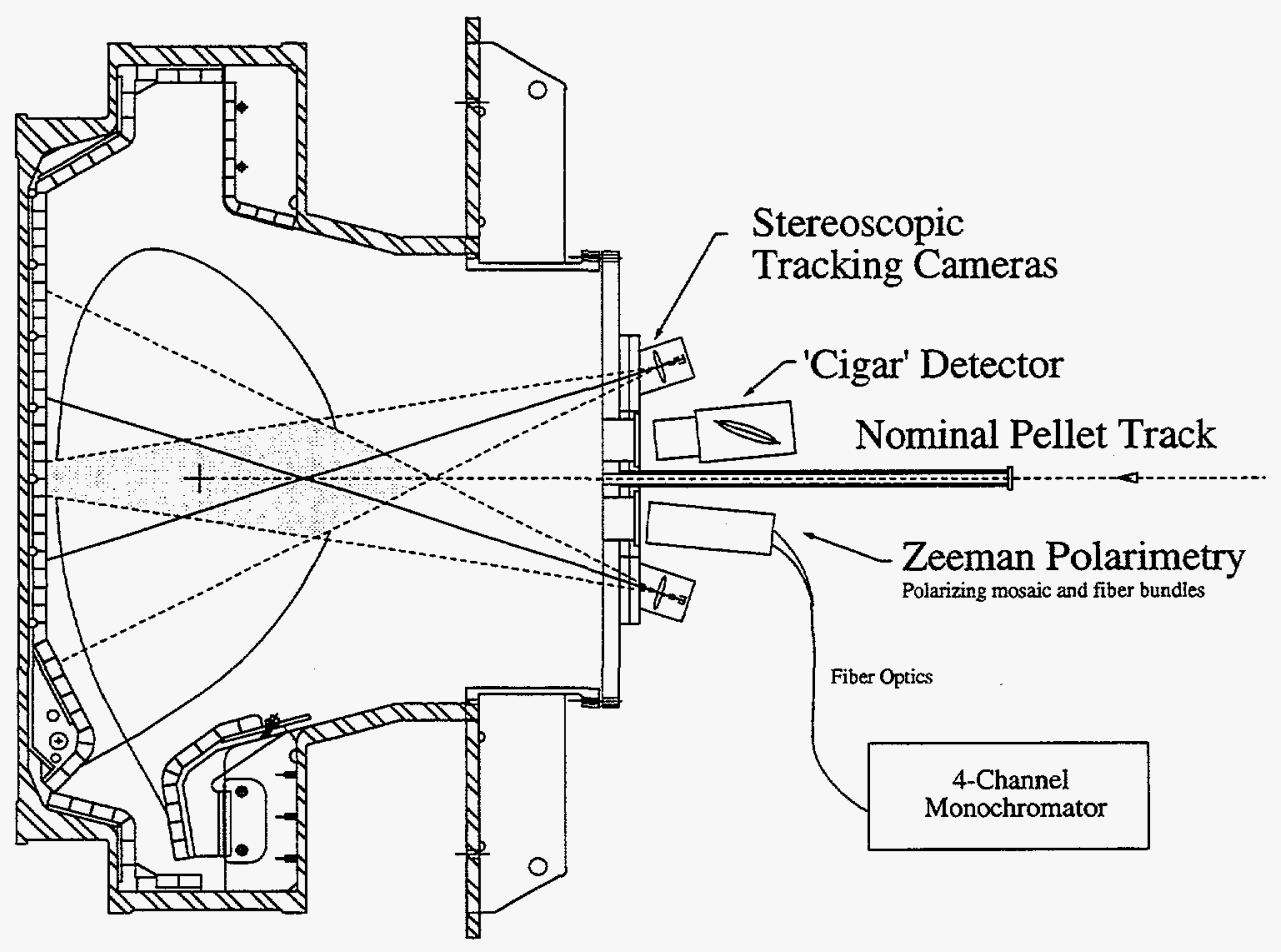

Figure 3.1: Lithium pellet diagnostics used to measure magnetic field tilt angle on the Alcator C-Mod Tokamak.

\subsection{Equilibrium reconstruction}

The equilibria presented in this chapter where obtained using a fitting code that solves the Grad-Shafranov equation. Known as EFIT $[11,12]$, this code was developed at General Atomics by L. Lao and coworkers and has been adapted for the Alcator C-Mod experiment by S. Wolfe. In this section a brief description of the code and external magnetic measurements used to fit for the plasma equilibrium is given.

\section{Magnetic Diagnostics}

Alcator C-Mod has an extensive set of magnetic diagnostics for equilibrium and instability measurement as was as real time control of plasma position and shaping.

The poloidal magnetic field, $B_{p}$, is measured in 26 different poloidal locations 


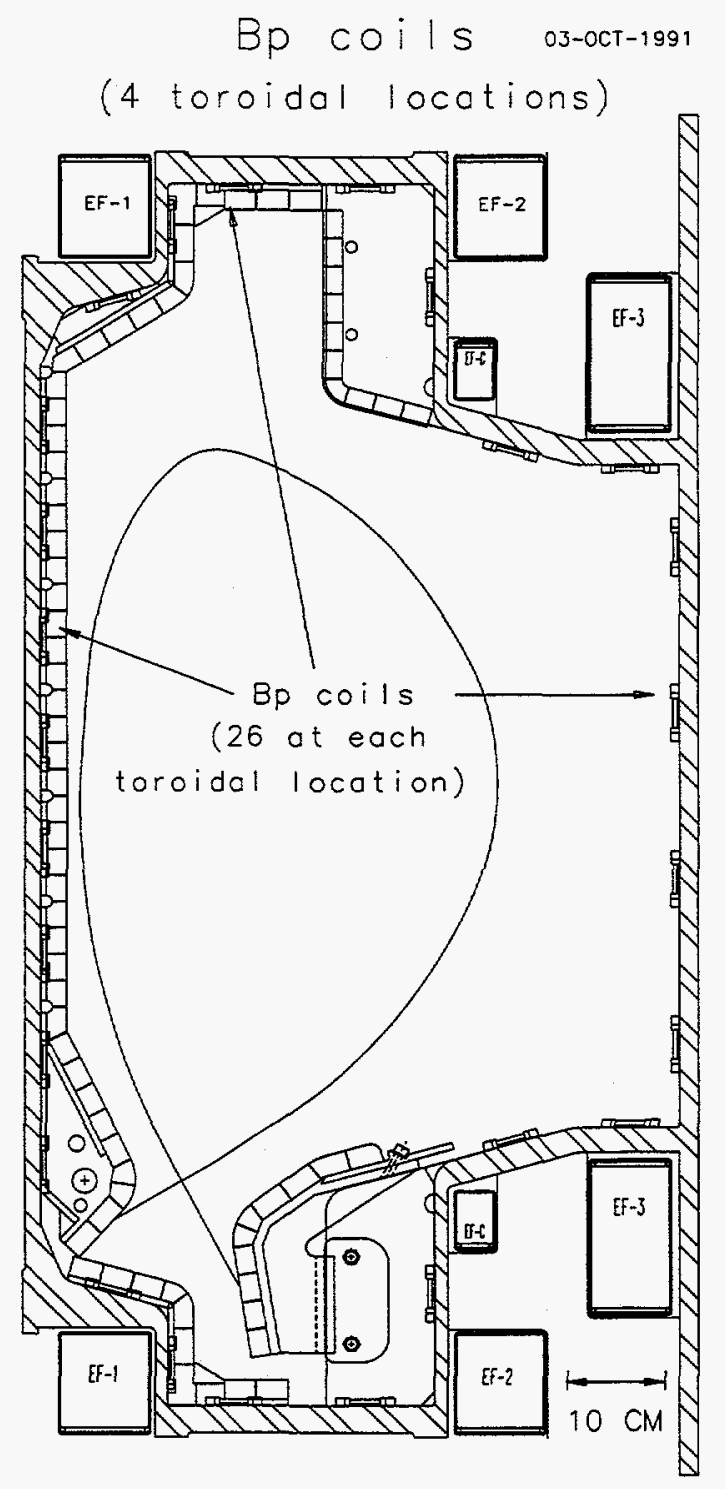

Figure 3.2: Arrangement of $26 B_{p}$ measurement coils mounted on the inside wall of the Alcator C-Mod vacuum vessel. There are four such sets at different toroidal locations. Interspaced between the $B_{p}$ coils are 26 full or partial flux loops that run toroidally around the machine. Other magnetic diagnostics include Rogowski coils for measuring the current within each magnetic coil as well as the total plasma current. 
using partial Rogowski coils mounted on the inside wall of the vacuum vessel. The placement of these $B_{p}$ coils is shown in Fig. 3.2. There are four sets of $B_{p}$ coils at different toroidal locations, allowing for redundancy in equilibrium fitting and plasma control, as well as allowing the toroidal mode structure of large MHD oscillations to be determined.

Interspaced between the $B_{p}$ coils on the vessel wall are loops of wire that run toroidally around the machine. These 26 loops measure the poloidal flux and hence called flux loops.

In addition to these equilibrium diagnostics, there are 15 full Rogowski coils that measure the current contained in the tokamak field coils as well as the total plasma current, $I_{p}$.

\section{EFIT}

EFIT uses an optimized iterative method (described in [11]) to fit solutions of the Grad-Shafranov equation (2.12) to experimentally measured values. In order to make the problem tractable, EFIT uses parameterizations of the flux functions $P(\psi)$ and $F(\psi)$.

In the nominal EFIT usage, low order polynomial expressions which go to zero at the plasma edge, are used for $P^{\prime}$ and $F F^{\prime}$. In addition, $q_{0}$ is constrained to be near unity. This technique of using external magnetic measurements alone gives reasonably accurate results for the equilibrium macroscopic quantities for elongated plasmas [13-15].

In order to reconstruct the details of the current density and $q$ profiles, internal magnetic measurements must be used to constrain the equilibrium reconstruction. For shots with abnormal current density or pressure profiles, more degrees of freedom must be used in the parameterization of $P(\psi)$ and $F(\psi)$ to accurately model the equilibrium profiles. In order for the reconstruction to remain well conditioned, 
the additional degrees of freedom in the equilibrium model require additional measurements to constrain the fit. These constraints are provided by the lithium pellet injection magnetic field tilt angle measurements to be discussed in the remainder of this chapter. For allowing yet further degrees of freedom in the equilibrium model, internal pressure measurements may also be used as constraints.

\subsection{Impurity Pellet Injector}

The Alcator C-Mod Impurity Pellet Injector is a versatile tokamak plasma diagnostic tool. As a current density diagnostic, the injector's primary role is to inject high speed $(\approx 1 \mathrm{~km} / \mathrm{s})$ lithium pellets into the center of the tokamak plasma. Secondarily, the impurity pellet injector may also be used to inject lithium or boron pellets for conditioning of the plasma first wall. Higher $Z$ impurity doped lithium pellets may also be injected to study core impurity transport or atomic spectroscopy. The injector has also been used to inject large high $Z$ pellets as "killer pellets". In these experiments high $Z$ elements, such as gold and silver, are used to rapidly radiate plasma stored energy to ameliorate the deleterious effects of plasma disruptions ${ }^{1}$ [16].

\subsubsection{Pellets: Size, Composition and Fabrication}

To be effective as a magnetic field tilt angle probe, the pellet must reach the place in the plasma where the measurement is to be made. While any projectile of sufficient size can penetrate to the plasma core, care must be taken not to disrupt the plasma. Thus the pellets must be of a relatively small size and injected at as high a speed

\footnotetext{
${ }^{1}$ Disruptions occur when control of the plasma is lost and much of the stored thermal and magnetic energy is absorbed into the tokamak structures. Large currents perpendicular to the magnetic field (called 'Halo' currents) can flow through the structures creating large forces, which can eventually lead to metal fatigue and failure. More benign, yet still of concern, disruptions also cause impurities to be reintroduced into the torus vacuum making it more difficult to achieve reliable breakdown and current rise.
} 
as possible to reach the plasma axis while minimizing the density rise caused by the pellet. A great deal of theoretical work has been done to model pellet ablation and predict the penetration depth [17-19]. Although the penetration depth depends strongly on the pre-pellet plasma electron temperature, a good empirical rule of thumb for the pellet size needed to penetrate to the axis of a fusion research tokamak is

$$
N_{e}^{p e l l e t} \approx N_{e}^{p l a s m a}
$$

In other words, the pellet must contain one plasma electron inventory. Thus, injection of a diagnostic pellet causes a significant perturbation of the plasma density and remains a large drawback to the entire technique. (As we shall see in Chapter 4, however, this perturbation is useful for other purposes.) For Alcator C-Mod, with a typical average electron density of $10^{20} \mathrm{~m}^{-3}$ and a volume of $\approx 1 \mathrm{~m}^{3}$, a lithium pellet with $10^{20}$ electrons $\left(\approx 1 / 2 \mathrm{~mm}^{3}\right)$ often penetrates just beyond the plasma axis.

Lithium was chosen as the pellet material for several reasons. It is the lowest atomic number $(Z)$ element, other than the main plasma species (deuterium), that may be fabricated into a pellet. Low $Z$ is important for two reasons. First, lithium becomes fully stripped in the post-pellet plasma and thus does not radiate large amounts of energy from the plasma core, which can be a cause of disruptions. Second, the plasma resistivity, which is proportional to $Z_{\text {eff }}$, the effective atomic number, $Z$, of the plasma, is less perturbed by injection of a low $Z$ element. Furthermore, lithium is a "low-recycling" impurity species and is observed in the plasma only directly following a pellet injection, leaving within the nominal particle transport time ( $\sim 20 \mathrm{~ms})$. Thus, the lithium does not remain in the plasma to increase $Z_{\text {eff }}$. In fact, lithium can actually improve tokamak cleanliness, and has been used on the graphite first walled TFTR [20] and DIII-D [21] tokamaks to reduce carbon and oxygen impurity concentrations. 


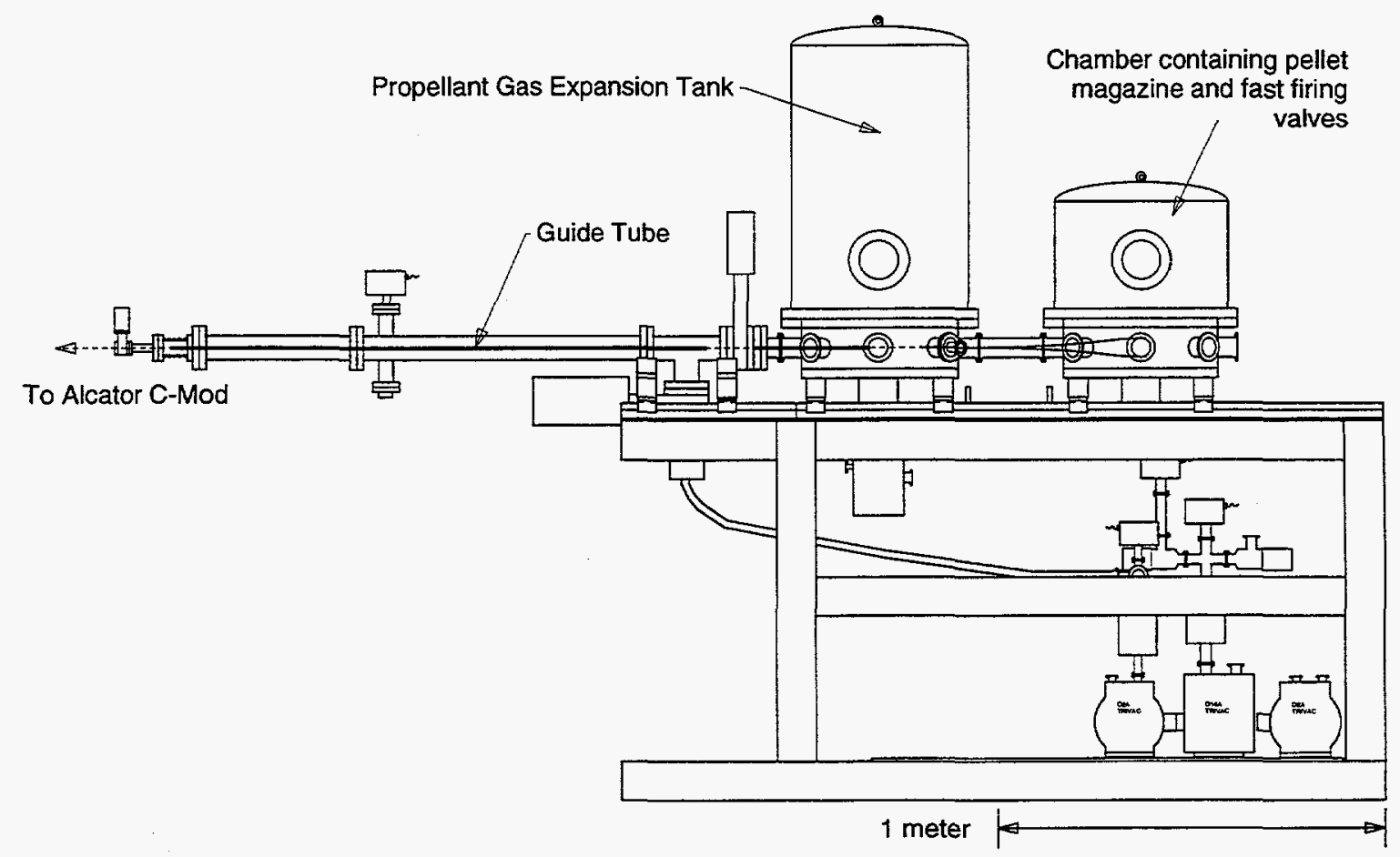

Figure 3.3: The Alcator C-Mod Impurity Pellet Injector.

Lithium pellets are fabricated in a "cookie cutter" fashion from lithium metal obtained in ribbon form. This is facilitated by the extreme softness and ductility of lithium metal. The cutters are small stainless steel hypodermic needles, the inner diameters of which define the diameter of the cylindrically shaped pellets. The length of the pellets is defined by the thickness of the foil from which the pellets are cut. Good injection reliability is obtained with pellets with length to diameter ratios of $1 / 3$ to $3 / 2$. Two different pellet diameters where chosen, .7 and $1.25 \mathrm{~mm}$, giving a size range of $N_{e} \approx .2-2 \times 10^{20}$ electrons.

\subsubsection{Injector Design}

The major elements of the impurity pellet injector, shown in Fig. 3.3, are a high pressure gas gun used to accelerate the pellets, a series of pellet catchers and guide 


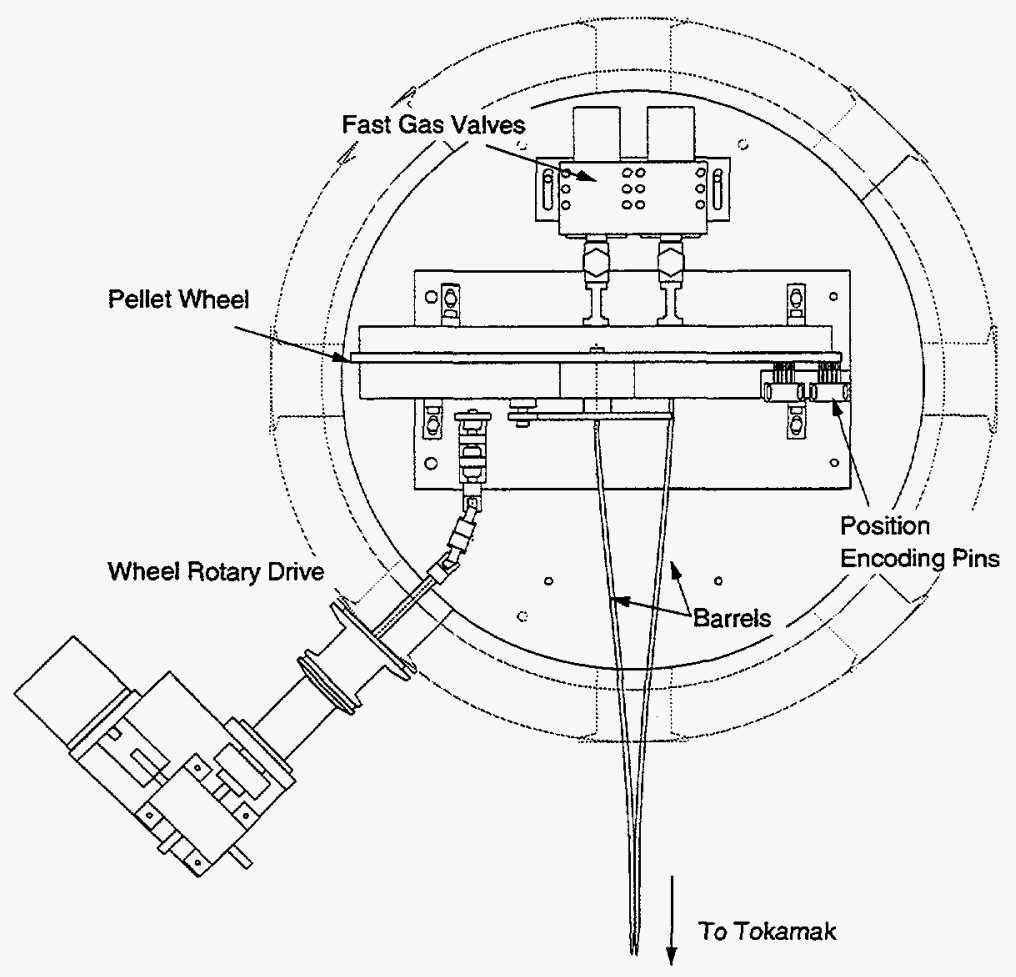

Figure 3.4: Top view of the single-gas gun that accelerates the pellets to $\approx 1 \mathrm{~km} / \mathrm{s}$.

tubes used to aim the pellets at the plasma, and two independent vacuum systems that serve to isolate the tokamak plasma from the propellant gas.

\section{Pneumatic Gun}

The pellet "gun" is a multi-barrel, single-stage, pneumatic gun with a revolving magazine. It consists of a set of four high speed, high pressure valves, a large rotary pellet magazine or wheel, and a set of four barrels in which the pellets are accelerated.

The fast valves were constructed following a solenoid design developed at ORNL. [22] They are capable of operation from 300-1000 psi, and are driven by transistor controlled capacitor banks that provide a 100A, 100V pulse. A typical pulse length used for reliable pellet injection, while limiting the total amount of gas released, is 
$\approx 1 \mathrm{~ms}$. The velocity of the injected pellet is determined primarily by the sound speed of the propellant gas. Because the highest speed possible is desired, hydrogen gas $\left(\mathrm{H}_{2}\right)$, with its low density and high sound speed, is used as the propellant. While changing the propellant pressure can also change the final pellet velocity, a quantitative study was not attempted and the pressure is typically (and somewhat arbitrarily) set to 650 psi.

The pellet magazine is a 14 inch diameter, $1 / 4$ inch thick, anodized aluminum disk, or wheel, in which a series of holes are bored. There are 4 rings of 90 holes or chambers, one ring for each barrel, for a total possible inventory of 360 pellets. Furthermore, a fifth ring of holes is provided for the rotational alignment of the wheel. The wheel is sandwiched between two plates in a vice-like manner. One plate connects through small tubes to the fast valves, while the other holds the gun barrels in place. The plate-magazine sliding interface is sealed with teflon o-rings at each barrel. The plates are aligned with large pins, slip-fitted on one side to allow the magazine gap, and thus the sliding friction of the seals, to be adjusted.

The location of the wheel axis is fixed by a shoulder screw into an axle that rotates in bearings mounted on the forward (barrel side) plate. This axle is rotated by a series of sprockets, chains, and other mechanical linkages from a stepper motor and reduction gearbox mounted outside the vacuum. When the magazine is rotated to a new chamber, alignment of the chambers and barrels is obtained by maximizing the light transmitted through the alignment ring holes and matching holes in the vice plates.

The absolute position of the wheel is indicated by a binary encoding laser-etched into the wheel's anodization. Small pins, connected to the front plate, slide along the surface of the wheel and are grounded when contacting an etched portion of the wheel. A 10-bit Gray Code encoding scheme, where only a single bit changes at each digital transition [23], was used to provide $.35^{\circ}$ absolute accuracy. Using this 


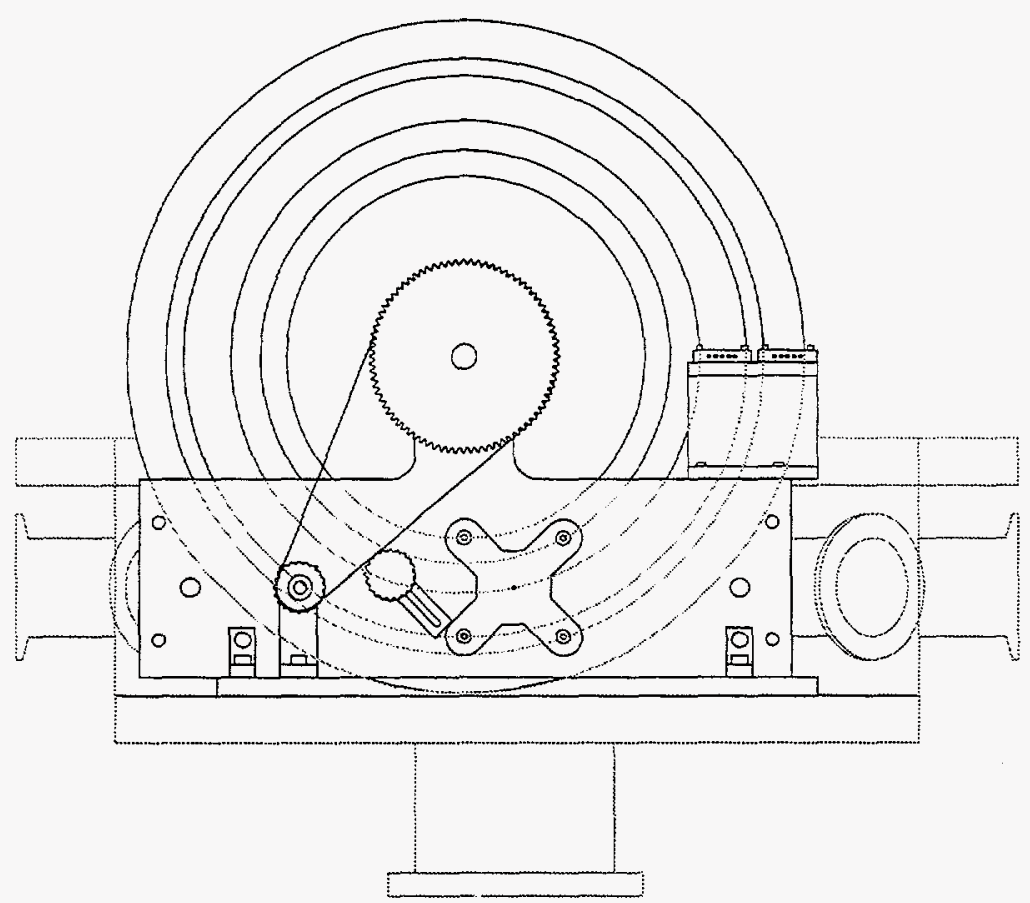

Figure 3.5: Front view of the gun showing the alignment of the barrels and the rings of holes in the pellet wheel magazine.

absolute position indication, a pellet inventory database is automatically maintained during injection operation.

The barrels themselves are also made from stainless steel hypodermic needle stock and are sized slightly larger $(\approx 0.1 \mathrm{~mm}$ larger inner diameter) than the pellets. The barrels are gradually curved and brought together parallel so that the pellets exit the barrels following roughly the same trajectory.

\section{Guide Tubes and TOF measurements}

After the pellets exit the barrels they enter and travel down small diameter $(5.3 \mathrm{~mm})$ "guide tubes". These tubes serve two functions. First, they transport the pellets to the tokamak and aim the pellets at the plasma axis. The guide tubes also make a very low conductance path for the propellant gases. 


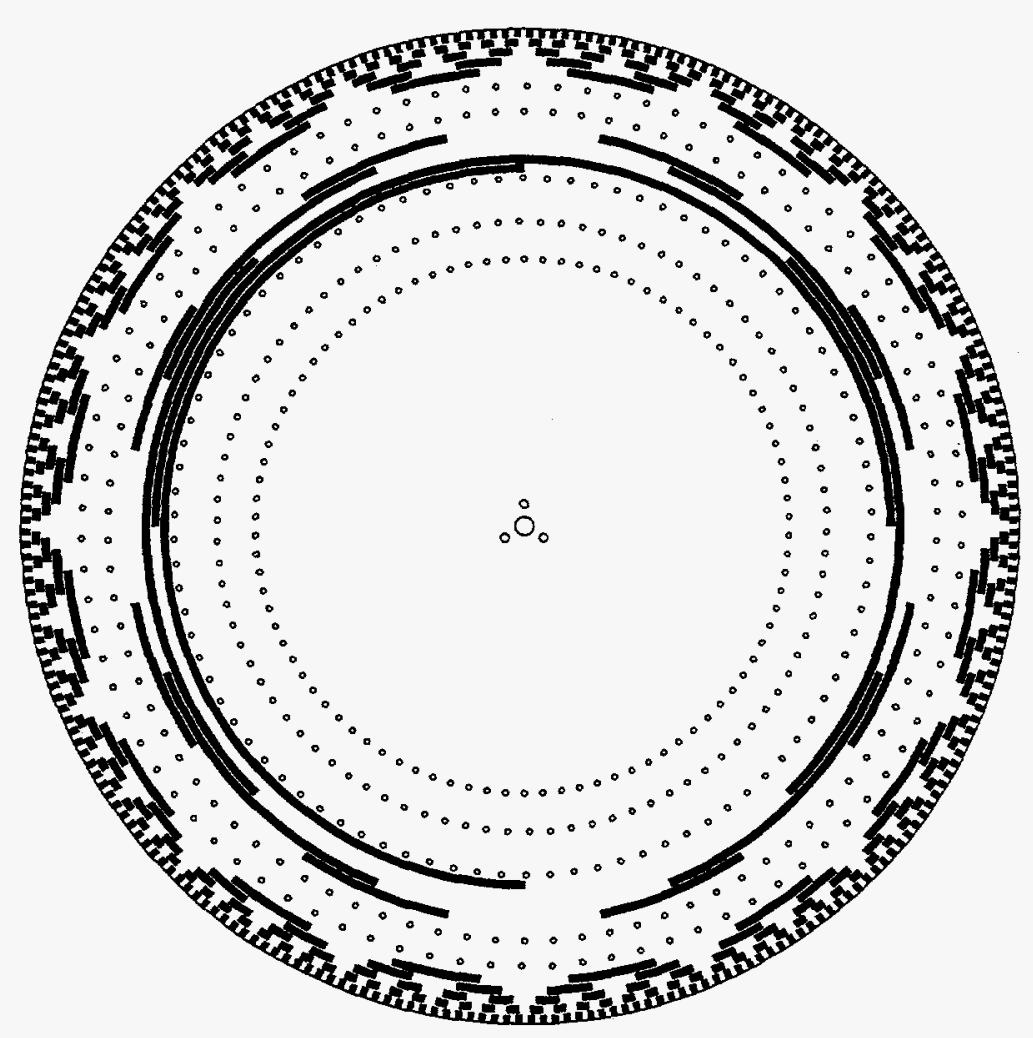

Figure 3.6: The pellet wheel showing the Gray Code rotary encoding used to help keep an inventory database.

As depicted in Fig. 3.7, there are numerous breaks in the guide tubes. One large break is made in each vacuum system to allow the propellant gas to expand into the volume of the vacuum vessel, dropping the pressure and reducing the gas throughput into the next vacuum system. After each gas expansion gap, the pellets are "caught" by shallow ( $1.5^{\circ}$ taper) funnels and then travel down the guide tubes.

Three photogates, which are interrupted by the passing of a pellet, are used to provide time-of-flight (TOF) speed measurements of the pellets. The use of three gates gives a measurement of three average velocities and an indication of the slowdown caused by the pellet glancing off the pellet catcher and guide tube walls. The photogates are made from expanded laser beams, aperatured with a narrow slit and collected onto photodiode detectors. Signals from these photodiodes are AC cou- 


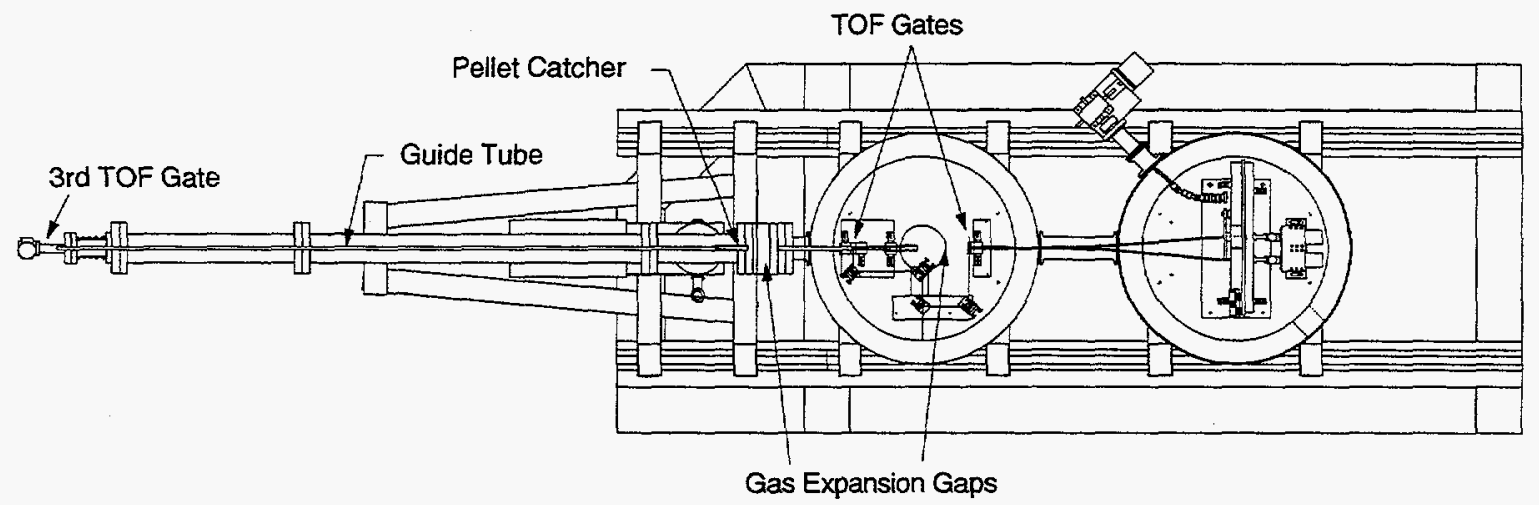

Figure 3.7: Top view of injector showing the guide tubes, time-offlight gates, and gas expansion free-flight gaps.

pled, amplified, and digitized at a $1 \mathrm{MHz}$ sampling frequency, giving roughly $5 \mathrm{~m} / \mathrm{s}$ resolution in the measured pellet speed.

\section{Vacuum and Propellant Gas Systems}

The impurity pellet injector vacuum system consists of two independently pumped and controlled vacuum subsystems. A plumbing schematic for these systems, as well as the propellant gas system, is shown in Fig. 3.8 .

The pellet gun and first set of guide tubes are contained within a large volume ( $\simeq 3501$ ) vacuum system designed to be of a relatively low quality (base pressure of $10^{-5}$ Torr), but easily serviceable. There are two stainless-steel bell jar vacuum vessels, joined by a short pipe, that make up this Lo Vac system. The smaller of the two bell jars houses the gun and provides easy access for the removal and reinstallation of the magazine wheel required for pellet reloading. The second, larger bell jar houses the first two TOF photogates and the first gas expansion gap between the end of the barrels and the first segment of guide tube. Because of this large volume, the pressure in this system reaches a maximum of just a few Torr after firing all four barrels of the gun. The Lo-Vac subsystem is pumped by a turbomolecular 


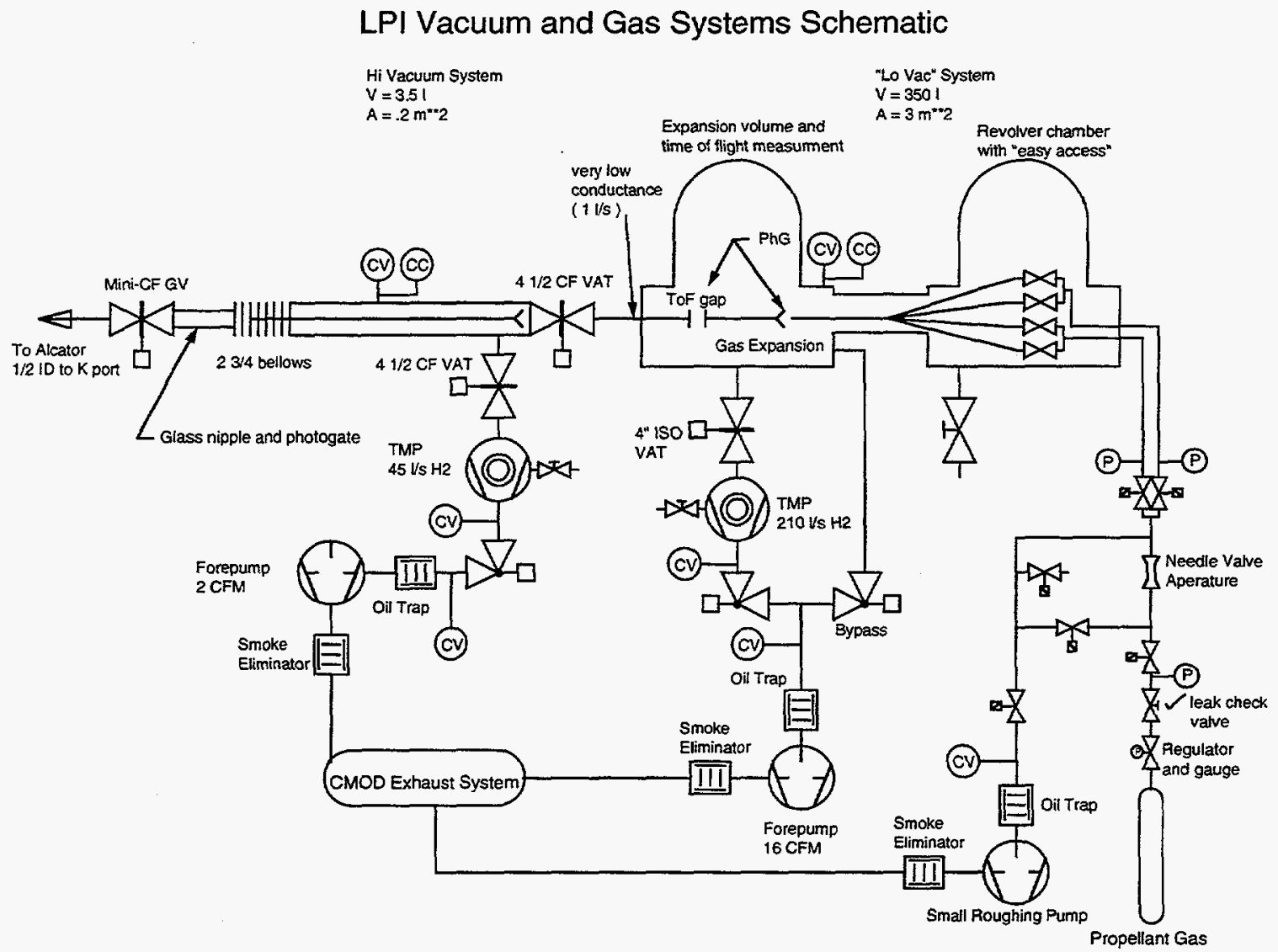

Figure 3.8: Schematic diagram of injector vacuum and propellant gas systems.

pump, capable of pumping $210 \mathrm{l} / \mathrm{s}$ of $\mathrm{H}_{2}$ at the base pressure, and backed by a $16 \mathrm{ft}^{3} /$ min rotary vane roughing pump.

The "Hi-Vac" subsystem, which consists of a much smaller volume, is the final buffer volume before the tokamak. As such, it is designed for a higher quality vacuum $\left(<10^{-7}\right.$ Torr base pressure) with bakable metal seals. It is connected to the Lo-Vac system only through the very low conductance $(<1 \mathrm{l} / \mathrm{s})$ guide tube. Furthermore, the two systems may be completely isolated by closing a gate valve that is located at the end of this segment of guide tube. The Hi-Vac system is pumped by a $45 \mathrm{l} / \mathrm{s}$ turbopump. This pump speed allows the steady-state pressure in the Hi-Vac system 
to be two orders of magnitude lower than that in the Lo-Vac. Thus, the post-pellet pressure rise in the Hi-Vac system is a few mTorr.

The last section of guide tube, which is actually a small diameter pipe that is welded directly onto the tokamak port flange (shown in Figure 3.1), provides the last low conductance path for propellant gas isolation. With this low conductance, and the large volume and pumping rate of the tokamak vacuum system, very little change $\left(<10^{-6}\right.$ Torr $)$ is observed in the base pressure of the tokamak when all four pellets are injected.

The high pressure propellant gas system is designed for pressures up to 1500 psi. The numerous valves and gauges in the system allow the pressures in two supply lines to be maintained and adjusted. The system can also be purged and pumped down to vacuum to ensure purity of the propellant gas.

The vacuum and gas systems are controlled by an Allen-Bradley programmablelogic-controller (PLC) computer programmed to provide automated fail-safe operation. The PLC is linked to a remote $\mathrm{PC}$, which provides a graphical user interface for manual operation and initiation of automated tasks, using Paragon control software.

\subsubsection{Performance}

The injector performance is quite satisfactory. Typical injection speeds for large diameter lithium pellets are $\sim 1250 \mathrm{~m} / \mathrm{s}$, while small diameter pellets have speeds approaching $1 \mathrm{~km} / \mathrm{s}$. Reliability of pellet injection is also very good. Neglecting operator errors (such as firing on a empty chamber), approximately $90 \%$ of all pellets requested reach the plasma. The only consistant hassle has been the manual loading of the pellet wheel, which can take several hours of tedium to complete. 


\subsection{Pellet Tracking System}

Both magnetic field tilt angle diagnostics make their measurements at particular times along the pellet trajectory. Thus, to reconstruct where these measurements are being made, accurate determination of the pellet position is required for the times of the tilt angle diagnostics. This is done using a system of two cameras employed stereoscopically to reconstruct the three dimensional position of the ablation light as the pellet transits the plasma.

\subsubsection{Stereoscopic Vision}

Tracking of an object using stereoscopic vision is a well understood problem. It requires two camera 'eyes' that provide signals to define a direction from the eye or camera lens to the object. A description of the cameras used in this experiment is given in the following section. Given the known location of these cameras, $\vec{L}_{1}$ and $\vec{L}_{2}$, (from an arbitrarily chosen origin) and the normalized direction vectors from the lenses to the object, $\hat{a}_{1}$ and $\hat{a}_{2}$, two lines of position are determined. The position of the object is, ideally, at the intersection of these lines. However, because of errors, either random or systematic, these two lines may not exactly intersect. So, to solve for the position of the object, we must find the position of the points of closest approach from one line to the other. The average of these points we will take to be the object position. The distance between these points will give us an estimate of the error in position (the 'cross-eyed' error).

Any point on these lines is described by

$$
\vec{C}_{i}=\vec{L}_{i}+\lambda_{i} \hat{a}_{i}
$$

First let us find the shortest chord, $\overrightarrow{C_{1}-C_{2}}$, between the two lines. It is easy to show 
that such a chord is perpendicular to both of the lines. Thus we must solve the set of equations

$$
\begin{aligned}
& \overrightarrow{C_{1}-C_{2}} \cdot \hat{a}_{1}=0 \\
& \overrightarrow{C_{1}-C_{2}} \cdot \hat{a}_{2}=0
\end{aligned}
$$

Solving for $\lambda_{1}$ and $\lambda_{2}$ and substituting $\vec{\Delta} \equiv \overrightarrow{L_{1}-L_{2}}$ for the camera lens separation we find that the object position is

$$
\begin{aligned}
\vec{C}= & \frac{\vec{C}_{1}+\vec{C}_{2}}{2} \\
= & \frac{\vec{L}_{1}+\vec{L}_{2}}{2} \\
& -\frac{1}{2} \frac{\left.\hat{a}_{1} \cdot \vec{\Delta}-\left(\hat{a}_{2} \cdot \vec{\Delta}\right)\left(\hat{a}_{1} \cdot \hat{a}_{2}\right)\right] \hat{a}_{1}+\left[\hat{a}_{2} \cdot \vec{\Delta}-\left(\hat{a}_{1} \cdot \vec{\Delta}\right)\left(\hat{a}_{1} \cdot \hat{a}_{2}\right)\right] \hat{a}_{2}}{1-\left(\hat{a}_{1} \cdot \hat{a}_{2}\right)^{2}}
\end{aligned}
$$

and the cross-eyed error is

$$
\left|\overrightarrow{C_{1}-C_{2}}\right|=|\Delta| \sqrt{1-\frac{\left(\hat{a}_{1} \cdot \hat{\Delta}\right)^{2}+\left(\hat{a}_{2} \cdot \hat{\Delta}\right)^{2}-2\left(\hat{a}_{1} \cdot \hat{\Delta}\right)\left(\hat{a}_{2} \cdot \hat{\Delta}\right)\left(\hat{a}_{2} \cdot \hat{a}_{2}\right)}{1-\left(\hat{a}_{2} \cdot \hat{a}_{2}\right)^{2}}}
$$

\subsubsection{Tracking Cameras}

Because the ablating pellets are very bright, the pellet tracking cameras are fairly simple devices. The very dense and relatively cold ablatant cloud immediately surrounding the pellet emits over $10 \mathrm{~kW}$ of bremsstrahlung and recombination radiation in the visible continuum. This emission is roughly 100 times brighter than the background plasma light. Thus, rather than trying to pick out the pellet in an image (as necessary in machine vision experiments), it is sufficient to determine only the position of mean brightness.

The measurement of the ablation position is accomplished by imaging the expected 
trajectory with a simple lens onto a two-dimensional position sensitive detector. This device used is a $1 \mathrm{~cm}$ square photodiode with resistive planes on front and back. The current produced in the diode is drawn from two electrodes on opposing edges of each resistive plane. As the resistive plane linearly divides the current between each electrode based on the distance of the current source to the edge, the mean position of the light on the detector is given by:

$$
x=\frac{i_{2}-i_{1}}{i_{2}+i_{1}},
$$

where $i_{n}$ is the current from each electrode and the position $x$ ranges from -1 at the first electrode to 1 at the other. As the resistive planes are oriented perpendicular to each other, both the $x$ and $y$ position of the imaged pellet are measured. Knowing this position, the direction of pellet from the camera is easily inferred.

Fig. 3.1 shows the position of each of these cameras on the tokamak and a crosssection (shaded in gray) of the volume where the pellets can be tracked simultaneously by both cameras. The signals from these cameras are amplified with transimpedance amplifiers and digitized at a $1 \mathrm{MHz}$ sampling rate with a CAMAC based digitizer giving approximately $1 \mathrm{~mm}$ resolution in the measured pellet position.

\subsubsection{Performance}

A sample pellet trajectory is shown in Fig. 3.9. The pellet travels through the magnetic axis, indicating the accurate aim of the injector and the full penetration of the pellet. (The magnetic axis is denoted by a + mark in the plasma cross-section and a dashed line in the other views. The dotted lined indicates the plasma boundary.) The pellet actually continues on to strike the inner wall (as indicated by the later observation of a puff of lithium into the plasma as the pellet bounces off the inner wall). However, the brightness of the pellet ablation cloud inside of $R \simeq 55 \mathrm{~cm}$ drops 

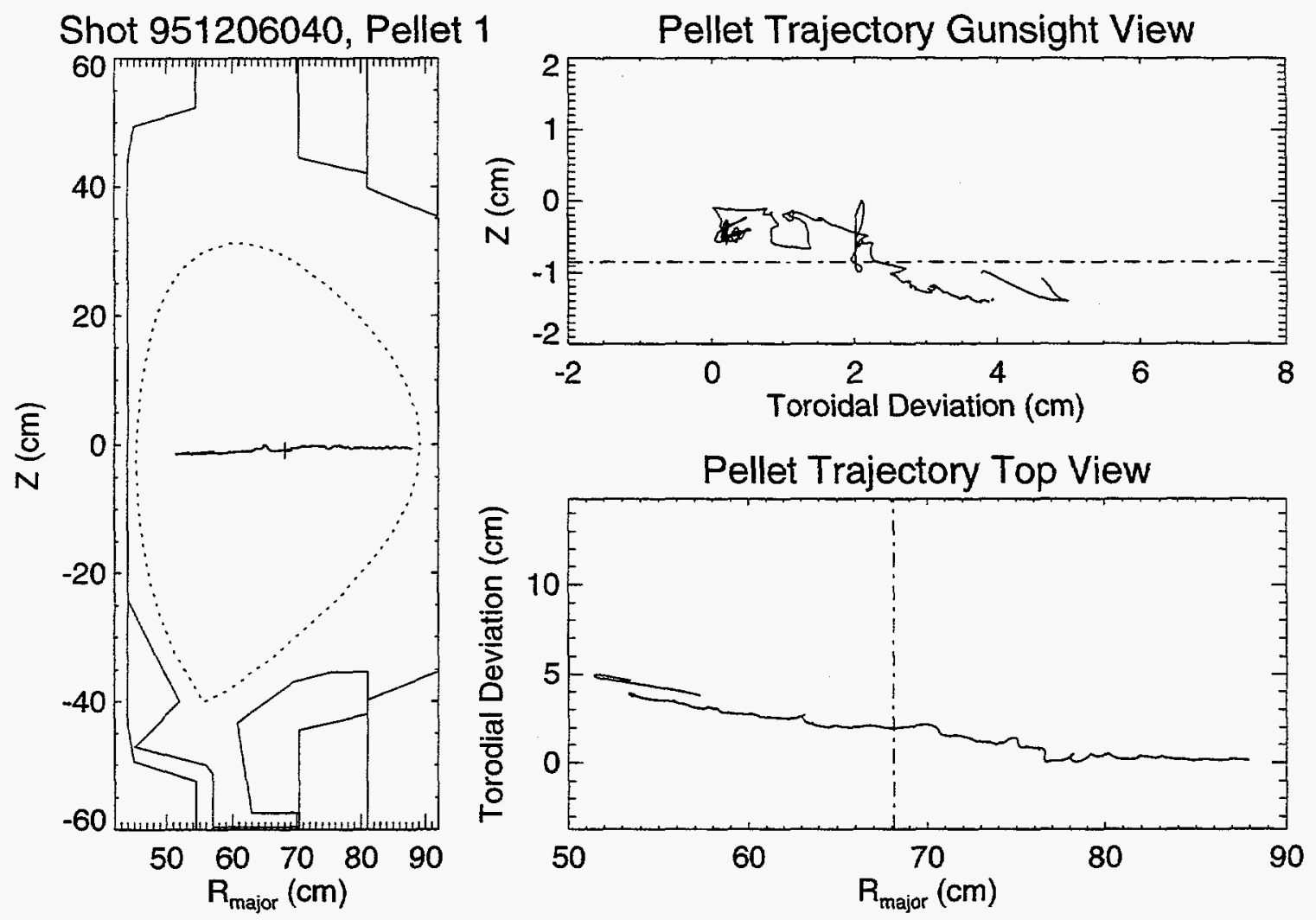

Figure 3.9: Example trajectory of pellet through plasma as determined from stereoscopic tracking system.

considerably while the background plasma light has increased because the pellet cools the plasma increasing edge radiation and core bremsstrahlung. This decrease in the relative brightness of the pellet invalidates the assumption used for the pellet tracking 'eyes'. The end result is that the cross-eyed error goes above $1 \mathrm{~cm}$ at which point the plot is terminated.

The $\approx 5 \mathrm{~mm}$ random deviations in the measured pellet trajectory, most easily seen in the gunsight view, are fluctuations in the position of ablation cloud mean brightness caused by instabilities driven by the steep density and temperature gradients near the pellet. These fluctuations are not indicative of the motion of the pellet. However, the large scale motion to the right and down, as seen in the gunsight view, is indicative of 
pellet motion. The curving of the trajectory indicates rocketing of the pellet caused by preferential ablation of the pellet on the side with the largest ambient heat flux. As the heat flux to the pellet is carried by the mobile electrons, the heat flux anisotropy is a result of the electron drift that makes up the plasma current. Thus, given a measured trajectory it is possible, in principle, to directly infer the plasma current density. In fact, a theoretical model [24,25], predicting pellet acceleration proportional to plasma current density, shows good agreement with carbon pellet experiments from the $\mathrm{T}-10$ tokamak. However, because the technique relies on determining the pellet acceleration, which is the second derivative of this somewhat noisy signal, meaningful results are difficult to obtain. The presence of a non-thermal hot electron population, from runaways ${ }^{2}$ or $\mathrm{RF}$ heating tails, further obscures the issue.

\subsection{Ablation Cloud Imaging Diagnostic}

This section describes the magnetic field tilt angle diagnostic referred to as the 'Cigar Detector'. The diagnostic relies on the experimental observation that the cloud of ablated lithium ions is spatially extended in one direction, or cigar-like in shape, as shown in Fig. 3.10. Since the charged ions are confined by the Lorentz force to move along the magnetic field, we conclude that the slope of the cigar is that of the local magnetic field lines. The technique of measuring the magnetic field tilt angle from the ablation clouds of deuterium and impurity pellets was first used on the TFR [26], T-10 [27] and TEXT [28] tokamaks and since been utilized on the TFTR [29] and ASDEX-U [30] tokamaks.

\footnotetext{
2 "Runaways" are a population of high energy electrons, often created in the early stages of the tokamak discharge when the density is low. Electrons are accelerated around the tokamak by the large initial loop voltage and as their velocity increases, the cross-section for collision and thermalization becomes vanishingly small. The electrons reach relativistic speeds and are indicated by the detection of hard x-rays created when the the runaways are lost and collide with a metal surface.
} 


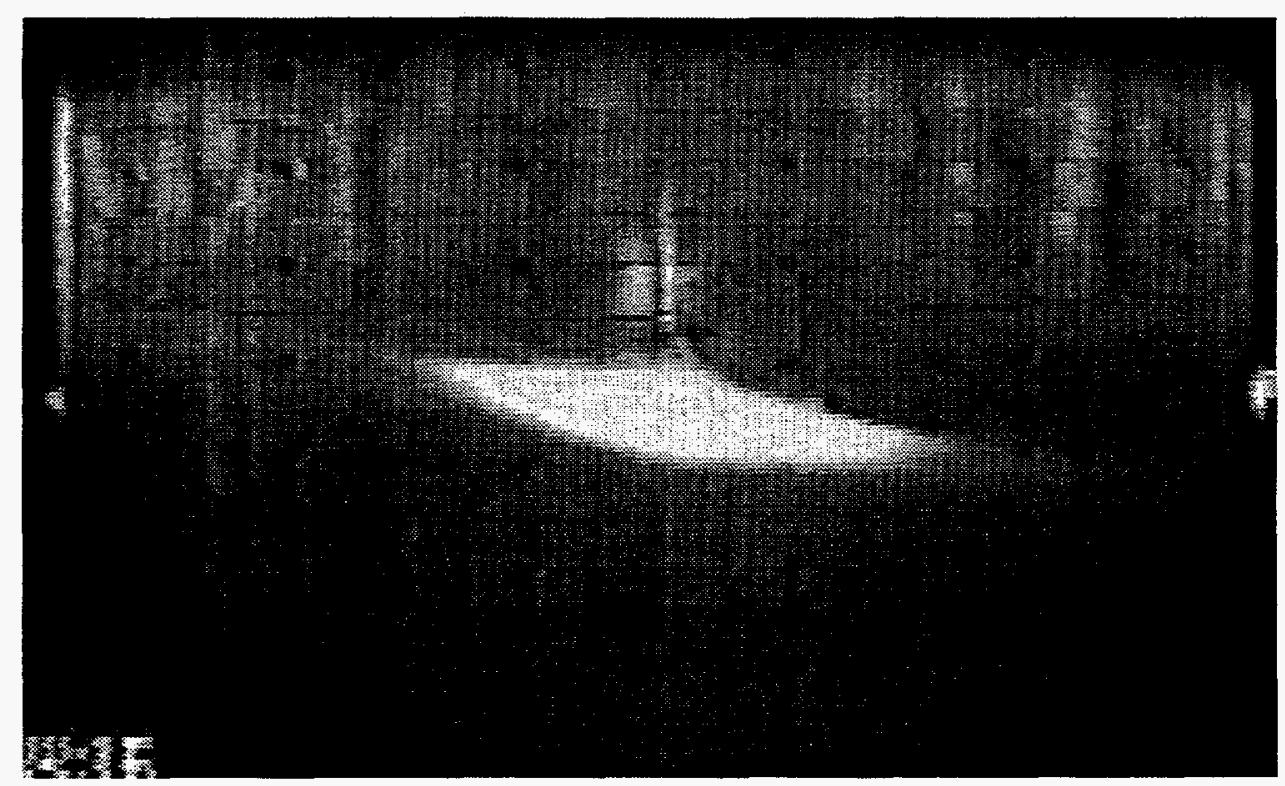

Figure 3.10: Gun sight view of pellet $\mathrm{Li}^{+}(5485 \AA)$ emission, time integrated over the entire pellet flight $(\approx 250 \mu \mathrm{s})$ using a standard CCD TV camera. The inner wall tiles are are made visible by edge plasma light during the $16 \mathrm{~ms}$ total exposure.

\subsubsection{Theoretical considerations}

Before turning to the implementation of this diagnostic, it is prudent to discuss the validity of the simplistic assumption that the observed pellet cloud is aligned along the magnetic field. To explore this issue it is necessary to understand the dynamics of the ablating pellet. The neutral gas shielding (NGS) model of pellet ablation, first introduced by Parks and co-workers [17], is depicted in Fig. 3.11. (A review of this model and its subsequent improvements is given in Milora et al [18].) The NGS model maintains that the pellet is surrounded by a dense sphere of neutral ablatant atoms that expand isotropically away from the pellet. This neutral gas cloud shields the pellet from the heat flux from the background plasma electrons, and thus, limits the ablation rate. Within the neutral cloud, as gas expands away from the pellet, the density drops (by at least $r^{-2}$ ) and the temperature increases until it is hot enough 

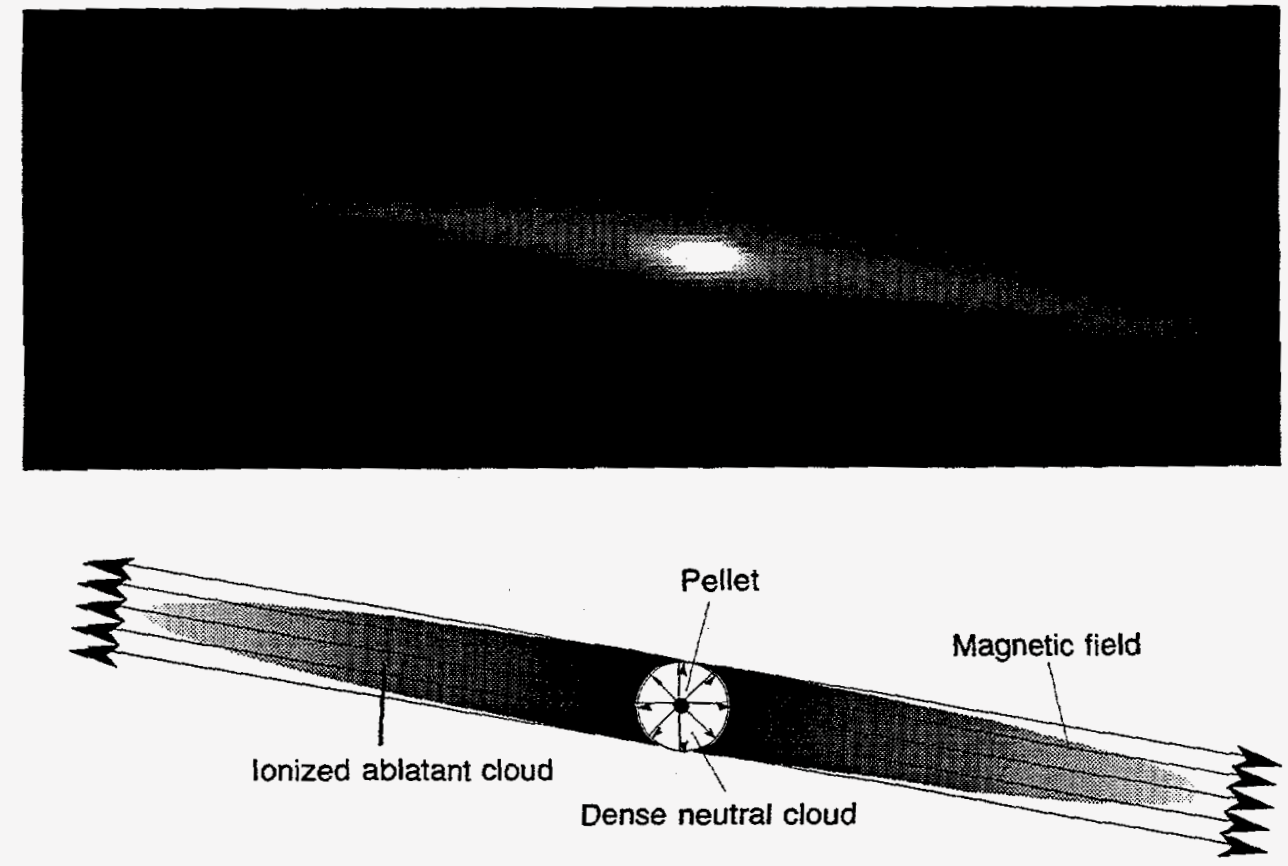

Figure 3.11: Upper: 250 ns snapshot of $\mathrm{Li}^{+}(5485 \AA)$ pellet ablation emission viewed from behind. The bright central spot is continuum emission from background plasma electrons slowing down on extremely dense partially ionized lithium ablatant gas. Lower: Schematic of neutral gas shielding model. 
to ionize the ablatant, at which point, the ablatant ions are confined to move along the field lines. The ions stream away from the pellet and are further heated by the background plasma, ionizing to higher and higher ionization states. While this model is based on a gross oversimplification of the environment surrounding the pellet, it is surprisingly effective in predicting pellet ablation and pellet penetration into fusion grade plasmas [31].

Spectroscopic measurements made on Alcator C [32] of Stark broadened neutral lithium emission indicate densities of up to $5 \times 10^{24} \mathrm{~m}^{-3}$ within the central cloud. Given that the ionization rate for neutral lithium (see Fig. 3.13) increases dramatically at electron temperatures $T_{e} \simeq 1 \mathrm{eV}$, an upper bound of $1 \mathrm{eV}$ within the cloud is taken. If these conditions are also true on Alcator C-Mod, with a typical magnetic field of 5.3 $T$, this implies a significant $\beta$ of $\simeq 10 \%$. The neutral cloud has a diameter of $\simeq 1 \mathrm{~cm}$, giving a transverse pressure gradient of up to $\sim 10^{8} \mathrm{~Pa} / \mathrm{m}$. Doppler broadened $\mathrm{Li}^{+}$ emission from outside the neutral cloud indicates temperatures of $\approx 15 \mathrm{eV}$. At these large pressures and densities, and with such large density and temperature gradients surrounding the ablating pellet, one might suppose that the ablation cloud is not aligned along the magnetic field.

One possible source of error is the diamagnetic exclusion of the magnetic field from the region of very high $\beta$. Such reduction of the magnetic field would have the effect of bending the magnetic field lines out and around the pellet. However, as the region of high $\beta$ is small and the exclusion effect is cylindrically symmetric, the only preferred direction is that of the unperturbed magnetic field. Thus, this effect causes no systematic perturbation on the ablation cloud. Furthermore, as the effect causes the natural field lines to expand outward near the pellet, and then return to their original position far from the pellet, this effect may even serve to shrink the thickness of the ablation cloud and increase the aspect ratio.

The extremely high densities of the ion ablatant cloud may present a larger prob- 
lem. For singly ionized lithium ions with temperature, $T_{i}=15 \mathrm{eV}$ and likely density of $n_{\mathrm{Li}^{+}}=10^{23} \mathrm{~m}^{-3}$, the classical ion-ion collision frequency is approximately 3 times greater than the ion cyclotron frequency. This implies that, at least initially near the pellet, the newly born lithium ions are not magnetically confined. However, this inequality is reversed for the electrons, which remain confined. Because the ions have high transverse conductivity, however, the ions move to shield the electric field from the cylindrical electron trail. Thus, the ions become electrostatically confined. Any ions that do diffuse away from the ablation cloud will tend to make the cigar width larger, and thus reduce the cigar aspect ration. Again, however, there is no preferred direction for these processes, other than the magnetic field, thus no systematic error is contributed.

The high transverse ion conductivity has other consequences. As shown in [19], in the rest frame of the moving pellet, the ions will move vertically to (partially) shield the Lorentz electric field $\mathbf{E}=\mathbf{v}_{\text {pel }} \times \mathbf{B}$. This creates a vertical polarization of the ion ablation cloud, which, when viewed in the laboratory frame, creates an electric field. This electric field produces an $\mathbf{E} \times \mathbf{B}$ drift with velocity slightly less than $\mathbf{v}_{\text {pel }}$. Thus, the ion ablation trail is dragged along with the pellet. This has the effect of decreasing the spatial resolution of the tilt angle measurement. However, because the pellet speed $\left(v_{\text {pel }} \approx 10^{3} \mathrm{~m} / \mathrm{s}\right)$ is much smaller than the ion flow speed, which is on order of the ion acoustic speed $\left(c_{s i} \geq 2 \times 10^{4} \mathrm{~m} / \mathrm{s}\right)$, this effect is overshadowed by the minimum spatial resolution set by the $1 \mathrm{~cm}$ width of the ablation cloud.

A similar effect was pointed out by Rozhansky et al [33]. The non-uniform magnetic field of a tokamak causes a vertical drift of ablatant ions and electrons (the $\nabla \mathbf{B}$ drift), creating a vertical electric field. This electric field then causes the ion ablation cloud to drift outwards in major radius. Although the polarization current is small even compared to the pellet velocity, because the ablation cloud is dragged along with the pellet, significant polarization can be achieved, accelerating the ablation 
cloud away from the pellet. This mechanism is unstable and can lead to fluctuations in the major radius of the ablation cloud. It is not clear how much error, if any, this adds to the tracker measurement of the cigar position. This mechanism may be responsible for the random accelerations seen on the pellet trajectory mentioned in $\S 3.3 .3$.

A possible influence on the tilt of the cigar with respect to the magnetic field would be poloidal rotation or flow of the plasma. A pellet injected into a plasma with a significant poloidal rotation would create a $\mathrm{V}$ shaped "wake" for an ablation cloud. However, no evidence for such bending of an ablation cigar has been found.

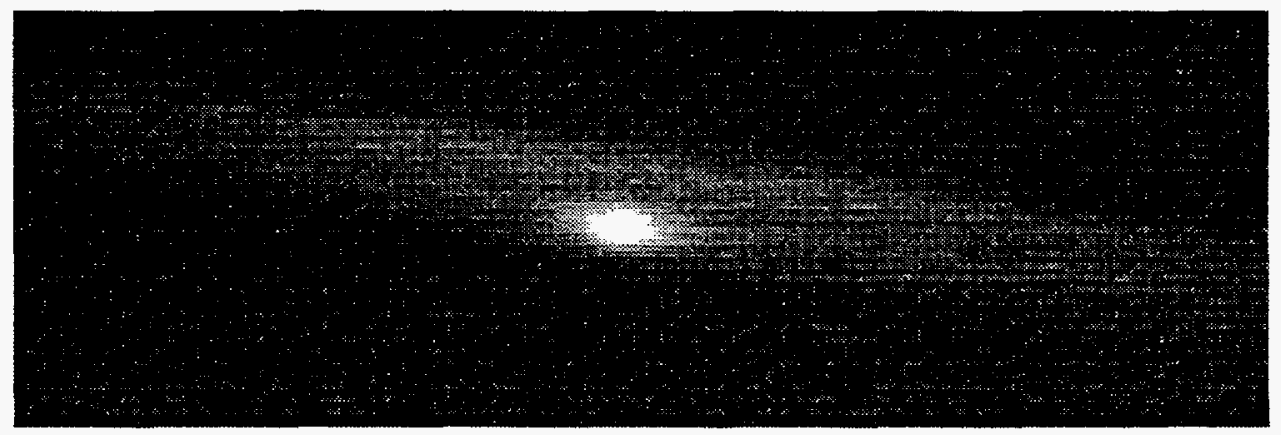

Figure 3.12: 250 ns snapshot of $\mathrm{Li}^{+}$ablation emission. Notice that ablation has filamenented into two distinct cigars. In the analysis, the upper filament, without the pellet and neutral cloud, is ignored.

So far, the ablating pellet has been treated as a uniformly expanding gas. However, the large gradients in density and temperature can also drive instabilities in the ablation dynamics or other collective effects in the ablation plasma that are not symmetric with respect to the pellet or the magnetic field. These fluctuations may lead to filamentation of the ablation cloud, such as shown in Fig. 3.12. They may also lead to random fluctuations of the ablation cloud tilt angle. Sometimes the imaged ablation cloud is clearly not cigar-like in shape and these images are disregarded in the analysis. In general, however, it is difficult to ascertain the size of the error that 
these fluctuations introduce in the tilt angle measurement. However, an estimate of the importance of these fluctuations will be given in $§ 3.4 .4$.

Finally, even if the assumption that the cigar is aligned along the local magnetic field is sound, there is the larger issue of whether the pellet injection disturbs the equilibrium field while penetrating the plasma. The density rise due to the pellet is large and this adiabatically cools the plasma. Furthermore, injection of large amounts of lithium raises $Z_{\text {eff }}$. Both lowering the temperature and raising $Z_{\text {eff }}$ have the effect of increasing the plasma resistivity, allowing the current density profile to change. However, this plasma current profile adjustment should be diffusive. Classically, this occurs on the magnetic field penetration time

$$
\tau_{B}=\frac{L^{2}}{\mu_{0} \eta}
$$

where $L$ is a characteristic scale length and $\eta$ is the plasma resistivity. Taking conservative values of $L=20 \mathrm{~cm}, T=100 \mathrm{eV}$, and $Z_{\text {eff }}=3$, gives a value of $\tau_{B}=15 \mathrm{~ms}$. This is nearly two orders of magnitude larger than the time it takes the pellet to reach the center of the discharge. Furthermore, the plasma will not be cooled to 100 $\mathrm{eV}$ until after the bulk of the pellet has been ablated in the plasma. Of course, current diffusion may be much greater than the classical value. However, double pellet experiments done on the TFTR with a second pellet injected $300 \mu \mathrm{s}$ after the first, showed no substantial change in the current density profile [34]. As we shall see in Chapter 4 , however, the pellet does measurably influence the current density profile on the timescale of $\sim 10 \mathrm{~ms}$.

\subsubsection{Ablation Cloud Aspect Ratio}

High cigar aspect ratio (the toroidal extension divided by the poloidal width) is important for lowering the experimental measurment of the magnetic tilt angle. Clearly 


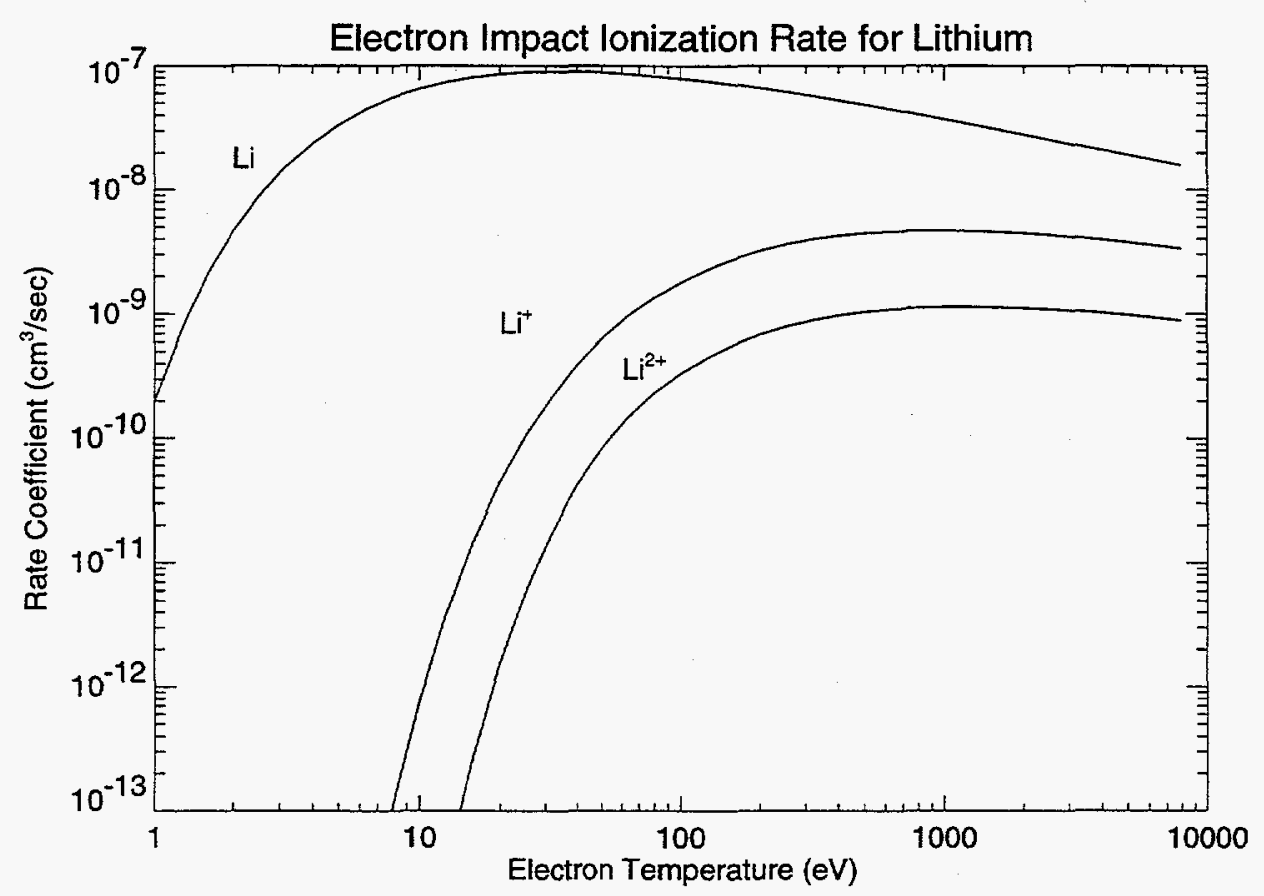

Figure 3.13: Electron impact ionization rates for Lithium atom and ions. After Bell et al [35]

a pencil-shaped ablation cloud is easier to measure than a one with a football shape. To maximize this aspect ratio, lithium turns out to be a good choice of ablatant material.

Shown in Fig. 3.13 are the electron impact ionization rates for the atomic and partially ionized species of lithium. The width of the cigar roughly corresponds to the ionization mean free path of the neutral atoms. Since Li-like lithium is relatively easy to ionize, this keeps the cigar width small. Contrarywise, He-like lithium, with its bound shell, is relatively difficult to ionize. Thus, the $\mathrm{Li}^{+}$ions have a long lifetime and travel significantly down the field lines before ionizing to higher states, giving a good aspect ratio. 


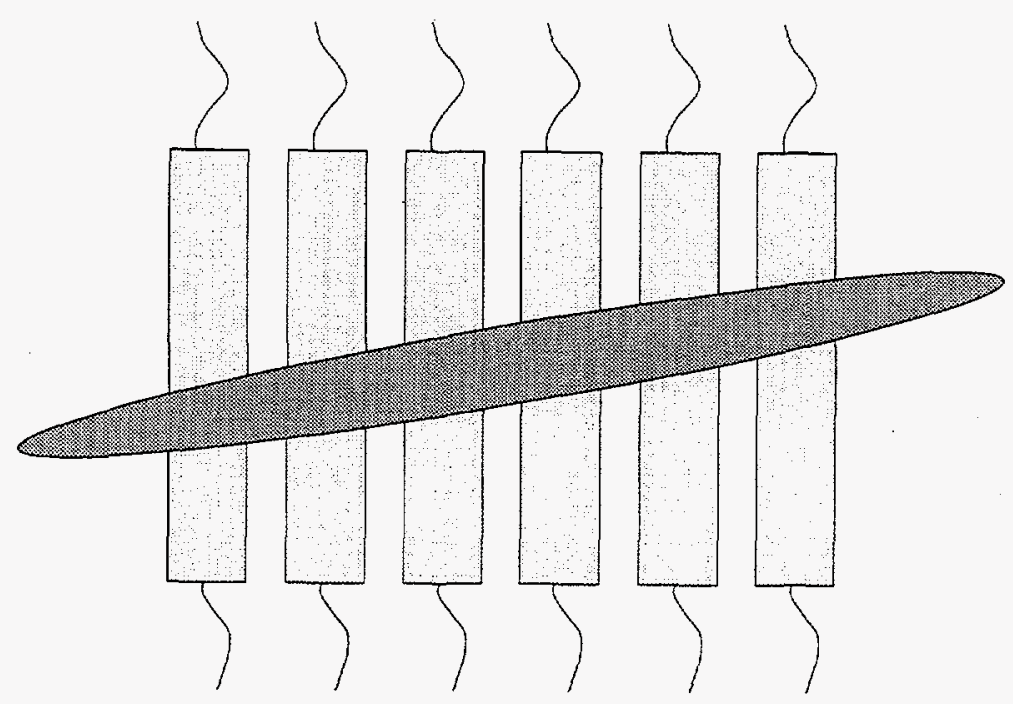

Figure 3.14: Schematic of pellet ablation cloud imaging diagnostic used on TFTR.

\subsubsection{The Cigar Detector}

To implement this diagnostic it is necessary to image the cigar-shaped emission onto a detector that is able to diagnose its tilt angle with the required temporal resolution $(\sim 10 \mu \mathrm{s})$. One such detector, that was employed with good results at the TFTR tokamak is a row of one dimensional position sensitive photodiode detectors (PSD) [29] shown in Fig. 3.14. As the response time of these detectors is $\sim 1 \mu \mathrm{s}$, this diagnostic allowed determination of the magnetic field tilt angle profile with excellent spatial resolution.

However, an implementation of this type of detector used on Alcator C-Mod suffered from problems of reflections and background interference because the PSDs detect the position of the imaged light as opposed to the position of peak intensity that corresponds to the cigar position. Furthermore, because of the high density of Alcator C-Mod discharges in comparison to other tokamaks, the toroidal extent of the cigar is shorter, making the cigar more difficult to diagnose.

Another detector, used on ASDEX-U [30], is a special CCD camera that effectively 
solves this problem. A high-resolution $(1152 \times 1024)$, slow-scan CCD detector is masked so that only every $51^{\text {st }}$ column of pixels is exposed. During operation the array is column shifted, moving each column of exposed pixels under the mask and exposing a new set of pixels. The column shift may be made every $2-50 \mu \mathrm{s}$, allowing 50 frames of $22 \times 1024$ pixels to be stored in as little as $100 \mu \mathrm{s}$. The array is then read out and digitized after the passing of the pellet. The camera is aligned so that the high spatial resolution is in the vertical direction and the peak position of the cigar in each of the 22 slices may be determined using robust fitting digital processing techniques. Measurements of the magnetic field tilt angle can be made with $\pm 5 \mathrm{mrad}$ accuracy. This approach is a good one and would have been attempted by the author if funds had been available.

On Alcator C-Mod, we have taken a simpler approach. Rather than make a specialized detector we use a high-speed gated CCD camera. This camera is a Xibion gated microchannel plate intensified RS-70 CCD. ${ }^{3}$ Although the camera is only capable of the standard 30 frames per second video, the intensifier can be turned on and off in less than $50 \mathrm{~ns}$, effectively shuttering the camera. The exposure gate is controlled by a programmable timing CAMAC module ${ }^{4}$, that is triggered by a comparator that samples the light intensity seen by the pellet tracking system. Thus, one or more snapshots of the pellet may be taken, with varying delay times, on the single video frame exposed during the pellet flight. Because it is difficult to distinguish multiple exposures, however, most data was taken with only one snapshot per pellet.

The images from the camera are stored on video tape and later digitized using a PC based 8-bit frame grabber and stored in GIF format. A direct to computer video system with recordable CD-ROM storage was also used on later experiments. The images are then transferred to the OpenVMS cluster workstations for analysis.

\footnotetext{
${ }^{3}$ Camera on loan from Dr. Glen Wurden of LANL.

${ }^{4}$ Jorway model J221/J222 timing pair.
} 
Two techniques have been used to determine the angle of these images, with similar results. The first involves enlarging the picture and manually picking several points that lie near the vertical center of the cigar. Depending on the cigar, this method is reproducible to within $\pm 0.1^{\circ}$. Concern that the manual method permits subjective weighting of the data lead to the development of a more automatic method. With this second technique, the image is rotated through a range of angles, and integrated horizontally at each angle to obtain projections of the pellet cloud intensity. These projections are then fitted to analytic curves whose peak heights and widths are well defined. Finally, by maximizing the peak value (or, if the ablation cloud projection is more trapezoidal, by minimizing the the fitted width) the cigar tilt angle is determined.

Because the absolute angle is required, the angle of the camera must also be determined. This was accomplished by opening the shutter and illuminating the inner wall of tokamak, which is covered by an array of small molybdenum tiles (see Fig. 3.10). Thus, using the manual technique, the angle of the camera may be inferred from the tile matrix. Repeating the measurement several times gives a value with a typical standard deviation of $0.16^{\circ}$.

\subsubsection{Cigar Detector Results}

As seen in Fig. 3.15, this pellet ablation cloud imaging method gives reasonable results and indicates that, typically, the EFIT equilibrium reconstruction, without any internal constraints, is very close to the experimentally measured values. Furthermore, the small scatter in the points indicate that the random error in the cigar detector measurement must also be small. 


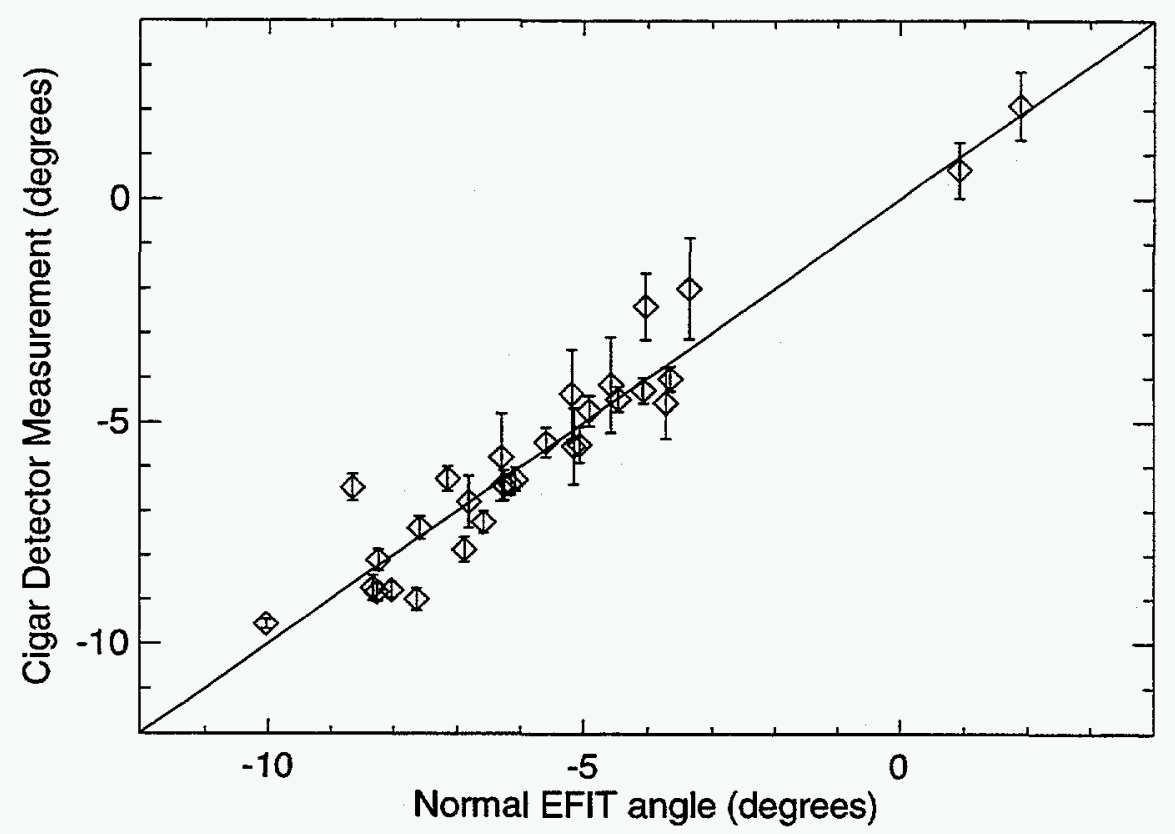

Figure 3.15: Measured cigar tilt angles versus the magnetic field tilt angles calculated from the standard (not internally constrained) EFIT equilibrium reconstructions of many different discharges. The cigar tilt angles were found using the automated fitting method. The error bars contain contributions from the cigar fitting and the tracking system positional uncertainties. 

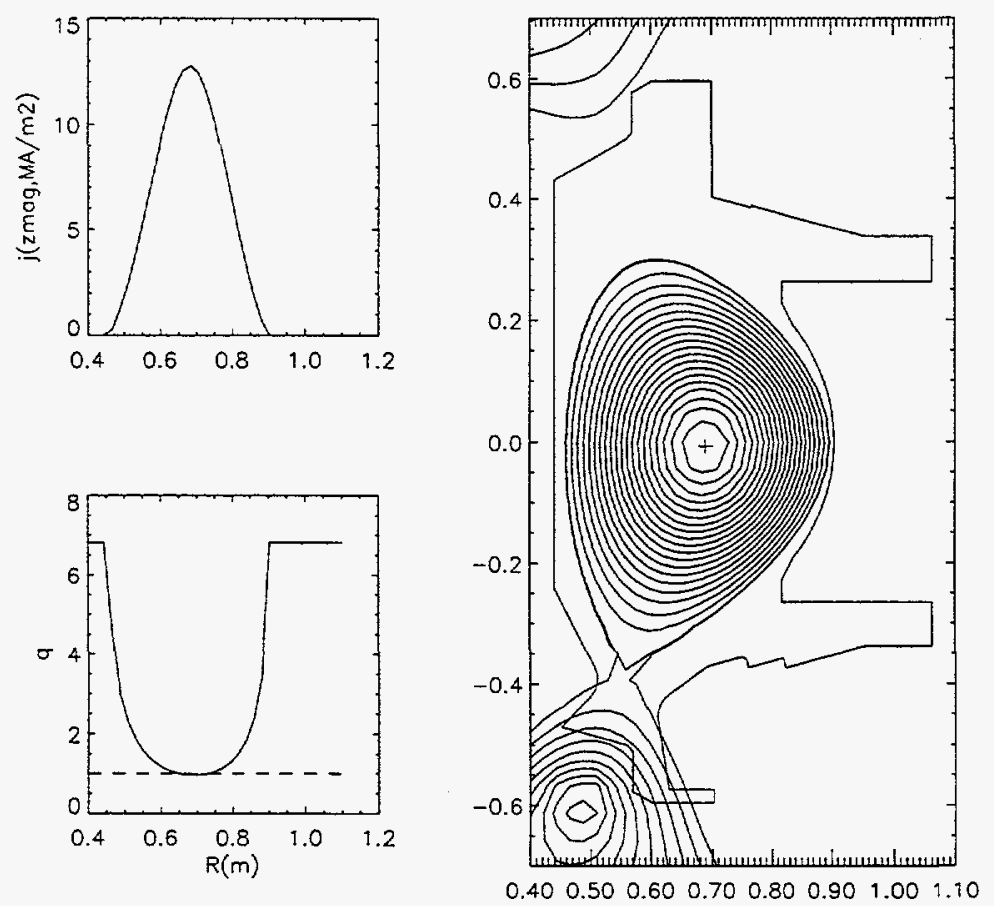

Figure 3.16: Normal EFIT reconstruction for of plasma equilibrium during a sawtooth suppressed PEP mode.

\subsubsection{Repetitive Shot Experiment}

The Xibion camera based cigar detector has one major drawback; it only gives one or possibly two magnetic field tilt angle measurements per pellet. Thus, it is not possible to obtain a radial profile of the magnetic field tilt angle with a single pellet. Therefore, in order to obtain the profile required to properly constrain the EFIT equilibrium reconstruction, it is necessary to repeat the experiment multiple times. This has been done for a discharge of particular interest: the sawtooth-suppressed pellet enhanced performance mode (which is the subject of Chapter 4).

Figure 3.16 shows the standard EFIT equilibrium reconstruction for discharge without internal magnetic field or pressure profile constraints. This reconstruction suggests that the value of $q$ goes below unity near the plasma axis. This is inconsistent 


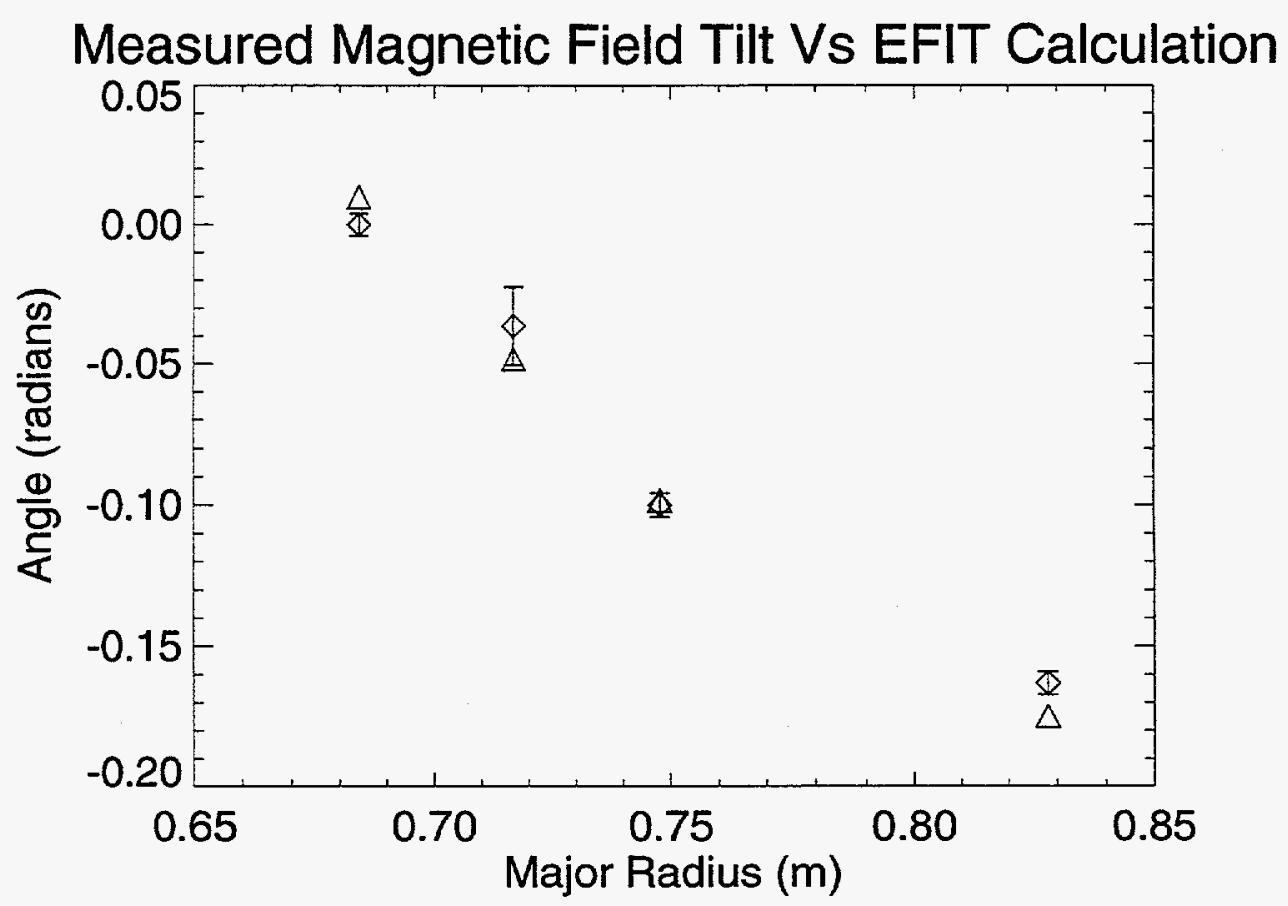

Figure 3.17: Comparison of cigar detector measurements $(\diamond)$ with tilts calculated $(\triangle)$ from EFIT reconstruction without internal constraints.

with the observation of sawtooth suppression during PEP mode discharges. (It is possible for sawteeth to be suppressed by high-energy non-thermal ions leaving $q_{0}<1$ [36]. However, there is no evidence for the presence of such a population in these discharges.)

Three good cigar measurements were made and combined with the position of the plasma axis, as determined from the tomographic reconstruction of the soft X-ray emissivity, to obtain a four point radial profile of the magnetic field tilt angle. This profile, compared with the values calculated from the unconstrained EFIT reconstruction, are shown in Fig. 3.17.

Constraining the EFIT reconstruction with the measured tilt angle data, we obtain the equilibrium shown in Fig. 3.18. Here we observe that $q$ is significantly above 

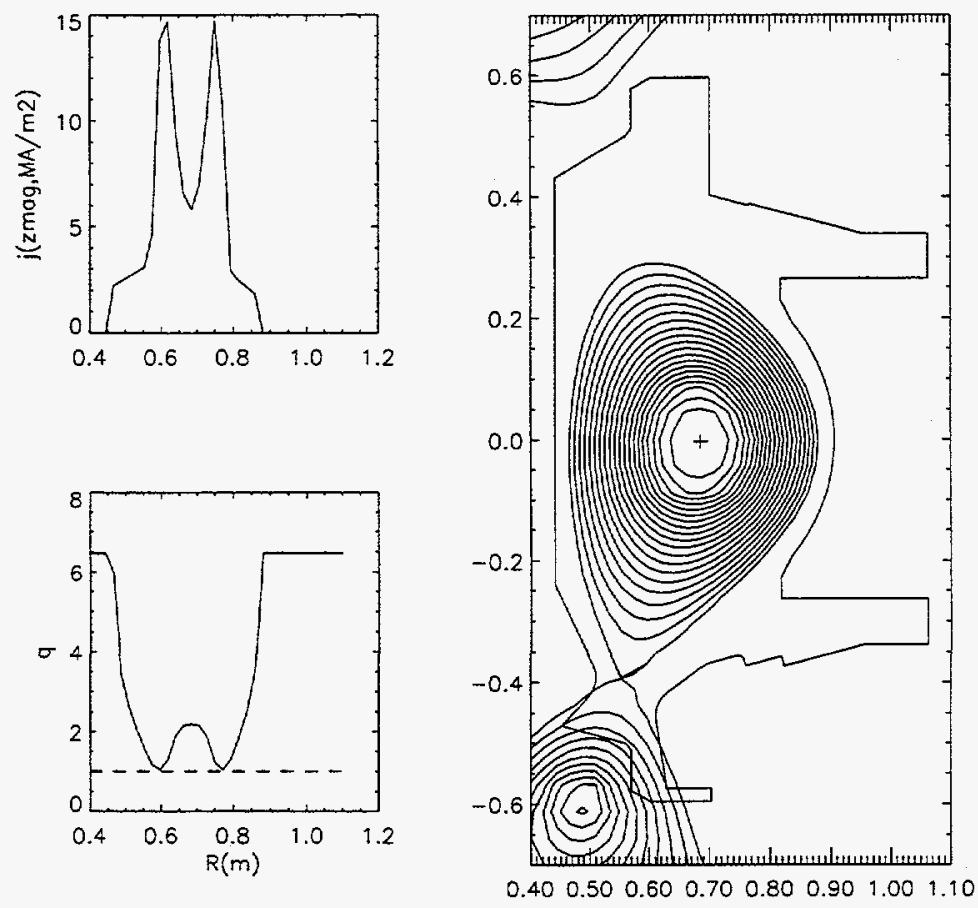

Figure 3.18: EFIT reconstruction using cigar detector data constraints gathered from a series of nominally identical shots. The data are taken during sawtooth suppressed PEP mode.

1 across the discharge, consistent with disappearance of sawtooth oscillation. Furthermore, this reconstruction demonstrates that a region of negative (or reversed) magnetic shear, $\hat{s} \equiv d \ln q / d \ln r$, exists in the center of the discharge. The corresponding fit to the measured tilt angle data is shown in Fig. 3.19.

One may question whether the reversed shear result persists when proper allowance is made for the given error in the innermost cigar measurement. Allowing fewer internal degrees of freedom for the EFIT reconstruction we obtain an equilibrium fitted to the limit of the error bar (in the direction of lower $q_{0}$ ), as shown in Fig. 3.20. Figure 3.21 shows the fit to the data.

As this possible equilibrium also has reversed shear, the cigar detector experi- 


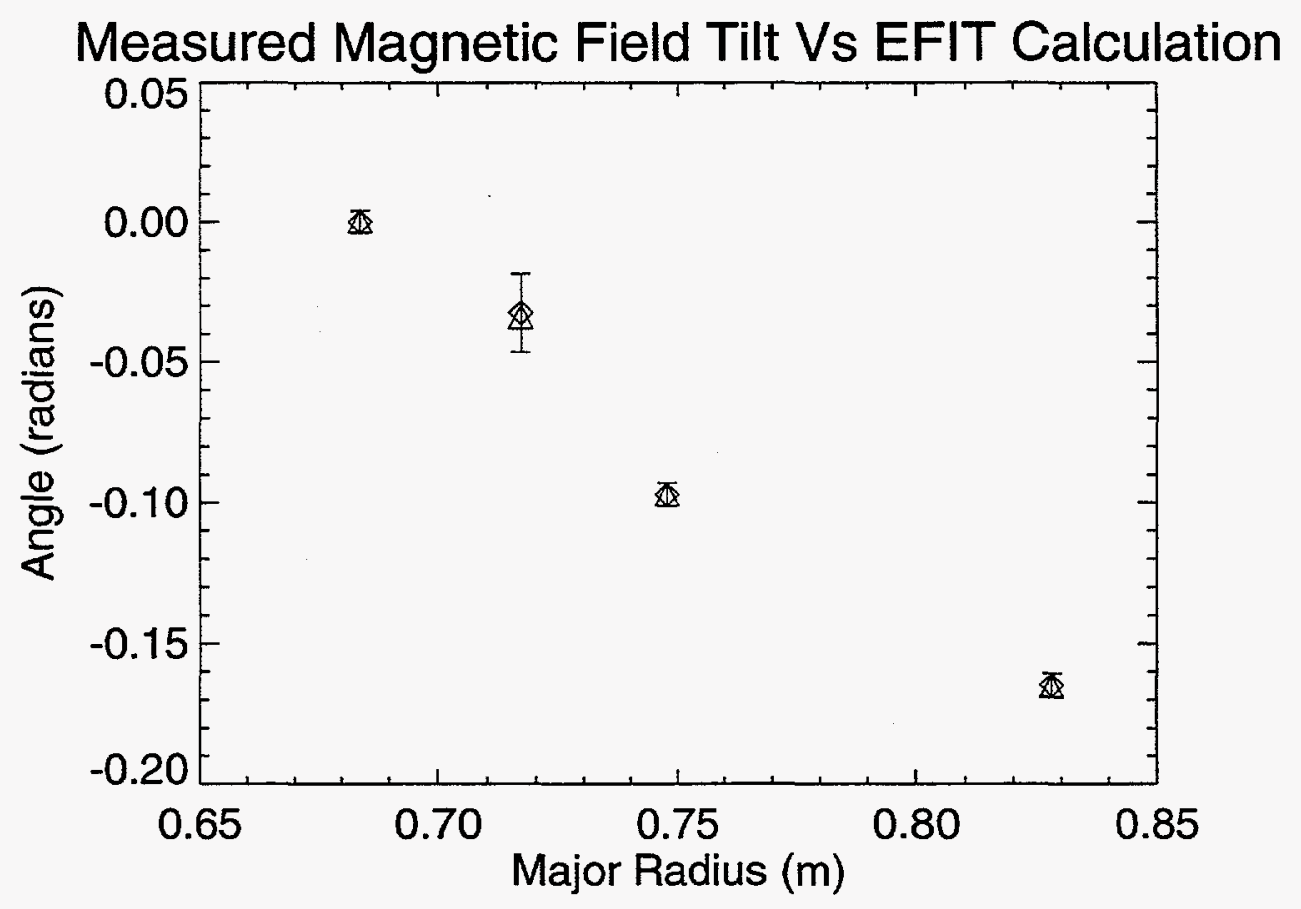

Figure 3.19: Comparison of cigar detector measurements $(\diamond)$ with tilts calculated $(\triangle)$ from EFIT reconstruction with constraints applied.

mental data demonstrates that, within the range of experimental error, PEP mode discharges contain a region of weak to moderate revered magnetic shear. The implications of this significant result will be discussed in Chapter 4 . 

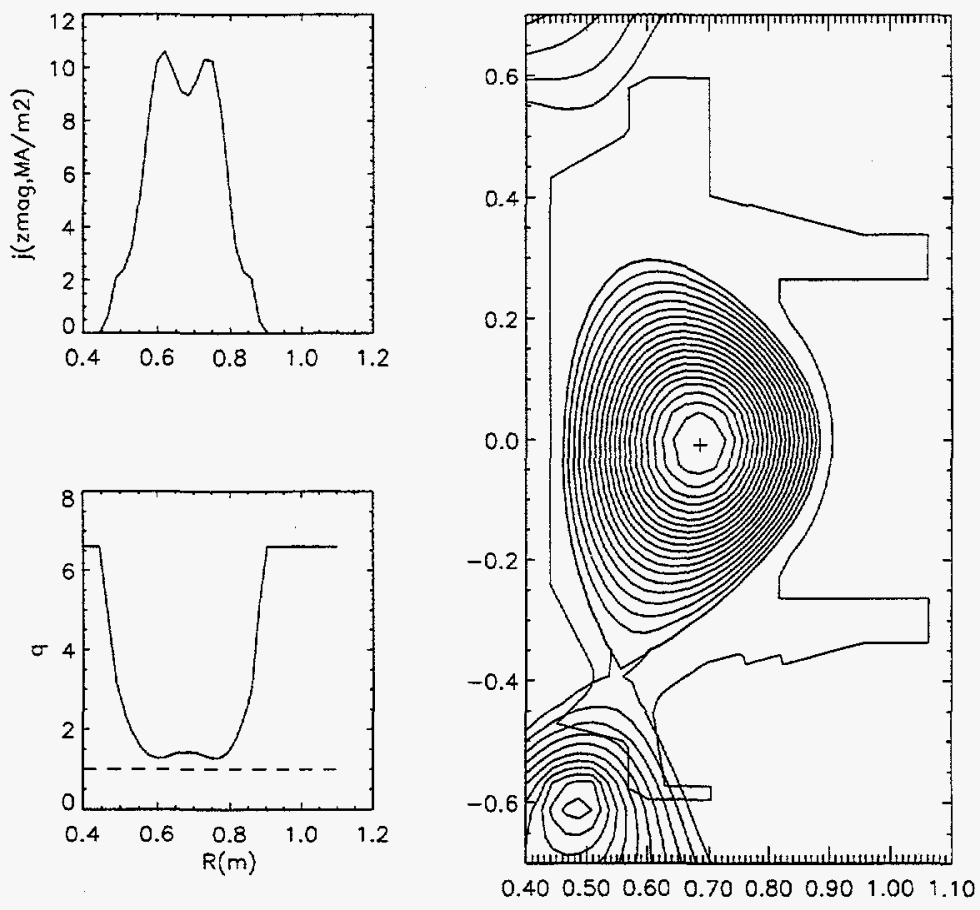

Figure 3.20: EFIT reconstruction using cigar detector data constraints fitted with constraints but less internal freedom. A region of weakly reversed magnetic shear is suggested. 


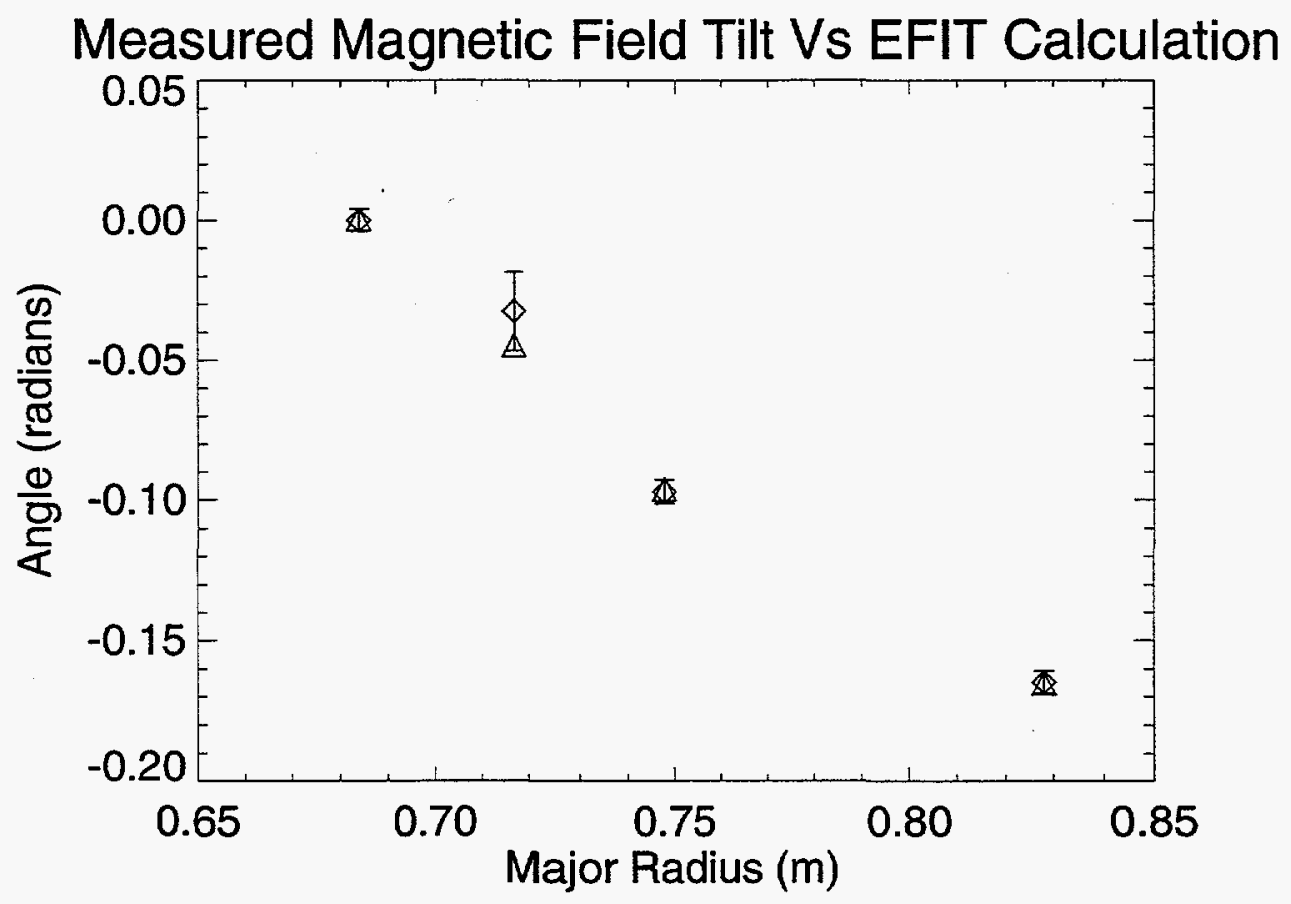

Figure 3.21: Comparison of cigar detector measurements $(\diamond)$ with tilts calculated $(\triangle)$ from EFIT reconstruction with less internal freedom. 


\section{Chapter 4}

\section{Pellet Enhanced Performance}

The injection of frozen deuterium and tritium pellets was first envisioned by Lyman Spitzer [37] in the early days of fusion research as a means to overcome the apparent difficulty of fueling a controlled fusion reactor. Classical transport theory makes no prediction for inward convection of plasma particles, and hence predicts no means of sustaining a density gradient. ${ }^{1}$ Fuel pellet injection would solve this apparent problem by providing a central particle source.

While early pellet injection experiments showed improvements in particle fueling efficiency, experiments on Alcator C [38] were the first to show that the discharge enters a mode of enhanced particle and energy confinement after the injection of deeply penetrating deuterium pellets. These modes, characterized by highly peaked density and pressure profiles, were demonstrated to have neoclassical-like core impurity accumulation and ion thermal transport with near neoclassical values. Termed

\footnotetext{
${ }^{1}$ Neoclassical theory, which takes into account the toroidal geometry of modern fusion devices, does, however, predict an inward convection of particles, the Ware pinch [8,9]. However, the Ware pinch is typically not large enough to explain the moderately peaked density profiles of the nominal L-mode discharge.
} 
'Pellet' or ' $\mathrm{P}$ '-mode, ${ }^{2}$ this mode of enhanced confinement was subsequently observed on numerous other devices, including ASDEX, DIII, PDX, TFTR, JFT-2M, JT-60 and others. The curious reader is referred to the review article by Milora et al. [18] for further references. More recently, experiments at JET $[39,40]$ have demonstrated that a region of reversed shear exists in the center of enhanced reactivity plasmas that develop after the injection of deuterium pellets with auxiliary heating. The term 'Pellet Enhanced Performance' or 'PEP' mode has been coined to describe these modes. This chapter explores the characteristics and modelling of PEP modes seen following the injection of lithium pellets on Alcator C-Mod.

\subsection{Introduction}

The phenomena discussed in this chapter occur following the injection of large lithium (or deuterium) pellets into a tokamak plasma (where $N_{e}^{\text {pellet }} \gtrsim N_{e}^{\text {plasma }}$ ). As previously mentioned, such large pellets injected radially typically penetrate to near or through the plasma magnetic axis.

Shown in Fig. 4.1 is pellet ablation light signal as detected by the pellet tracking system as a function of time during the pellet flight. Assuming that the ablation light is proportional to the pellet ablation rate $\left(d N_{e} / d t\right)$, we may normalize the integral of the ablation light signal to the total number of electrons within the pellet to obtain the electron deposition rate. This assumption is partially substantiated by the small observed variance of this normalization factor for a large range of different pellet sizes and penetration depths. Mapping the electron deposition rate to major radius using the pellet trajectory measurement and taking the volume contained in each flux

\footnotetext{
${ }^{2}$ The choice of this name was a natural one considering the convention of referring to a mode of confinement based on a single letter. For example, a mode of enhanced confinement observed on every diverted tokamak is called 'H-mode'. Here ' $\mathrm{H}$ ' stands for 'High' as to distinguish it from the nominal ' $L$ ' or 'Low' mode. However, the name 'P-mode' has not been very popular due to its unpleasant connotation in English slang.
} 


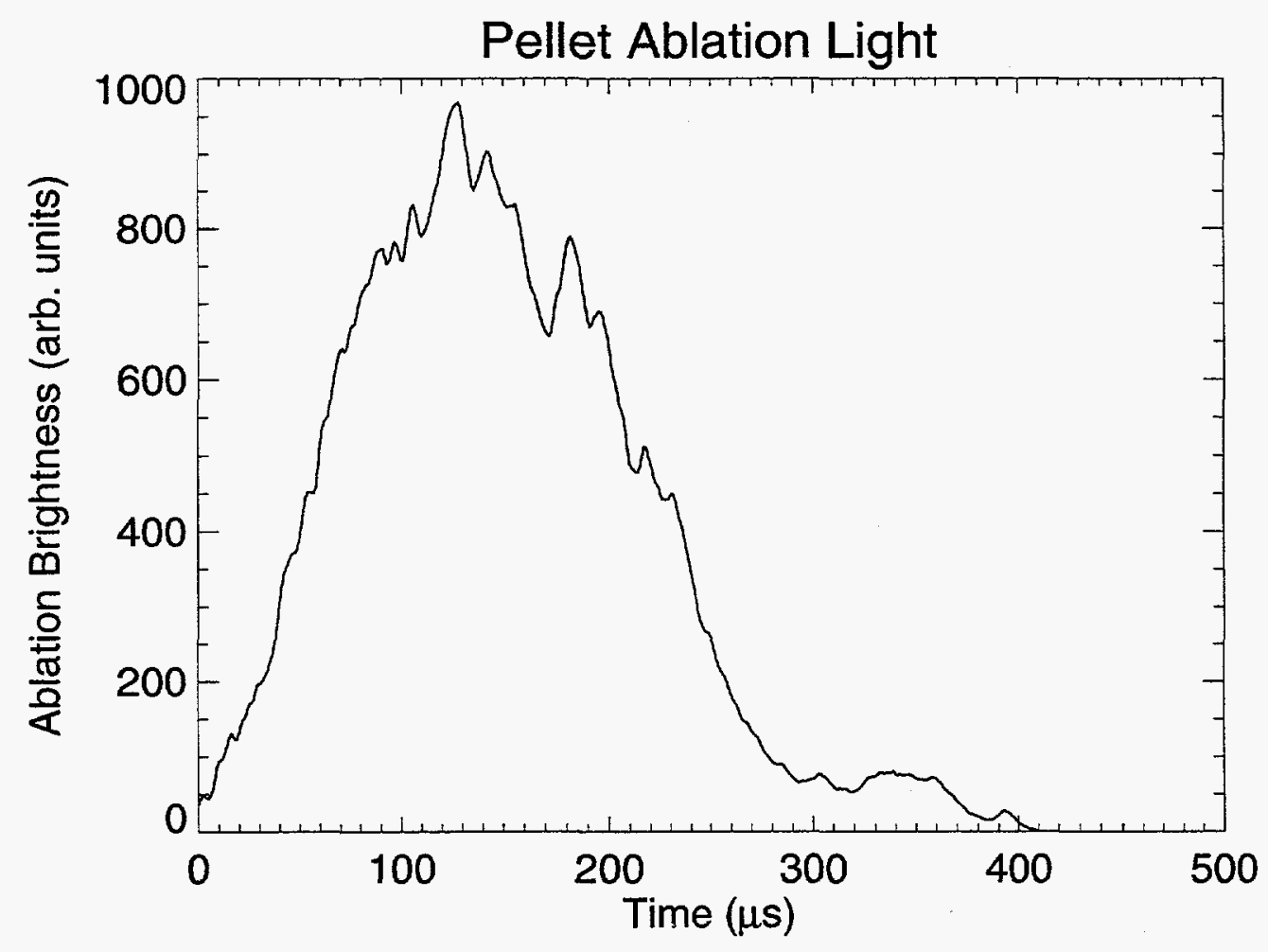

Figure 4.1: Visible ablation light signal from pellet injected into plasma.

cylinder into account, we obtain the expected electron density increase caused by the pellet injection. This profile is shown in Fig. 4.2. Note the extremely high peakedness of this profile in comparison to the nominal pre-pellet density profile. While the immediate post-pellet density peakedness is most likely reduced by fast anomalous particle transport during the pellet flight, the measured density profile (taken nearly $20 \mathrm{~ms}$ after the pellet injection) shows good agreement with the highly peaked density deposition profile. It is not surprising, therefore, that such core penetrating pellet injections have a dramatic effect on the post-pellet plasma. 


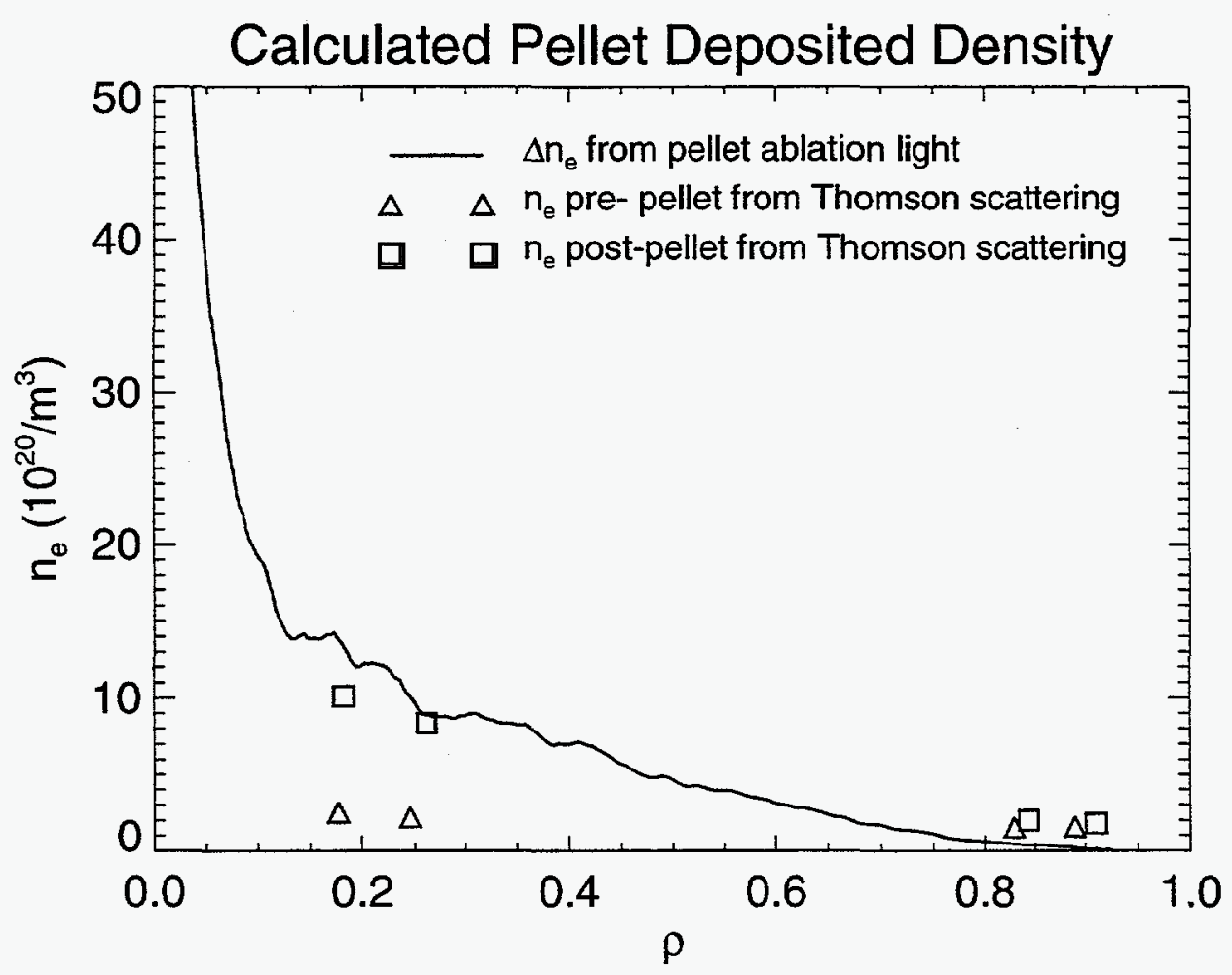

Figure 4.2: Expected electron density rise as a result result of pellet injection compared to pre- and post-pellet density measurements. (Shot 950609013.)

\subsection{Observed Characteristics of PEP mode}

Figure 4.3 shows the effect of a large pellet injection and some of the easily observed characteristics of a PEP mode discharge. During this discharge two 2 MW ICRF pulses were applied. At $0.5 \mathrm{~s}, 30 \mathrm{~ms}$ before the first ICRF pulse, a large lithium pellet was injected into the plasma, penetrating through the magnetic axis and doubling the line averaged electron density. The rapid increase in density adiabatically cools the plasma to cause the observed concomitant drop in electron temperature. ${ }^{3}$ Prior

\footnotetext{
${ }^{3}$ The electron temperature signal shown in Fig. 4.3, with data missing from 0.5 to $0.56 \mathrm{~s}$, is measured from O-mode second harmonic electron cyclotron emission (ECE) which is cut off during the high density post-pellet plasma.
} 


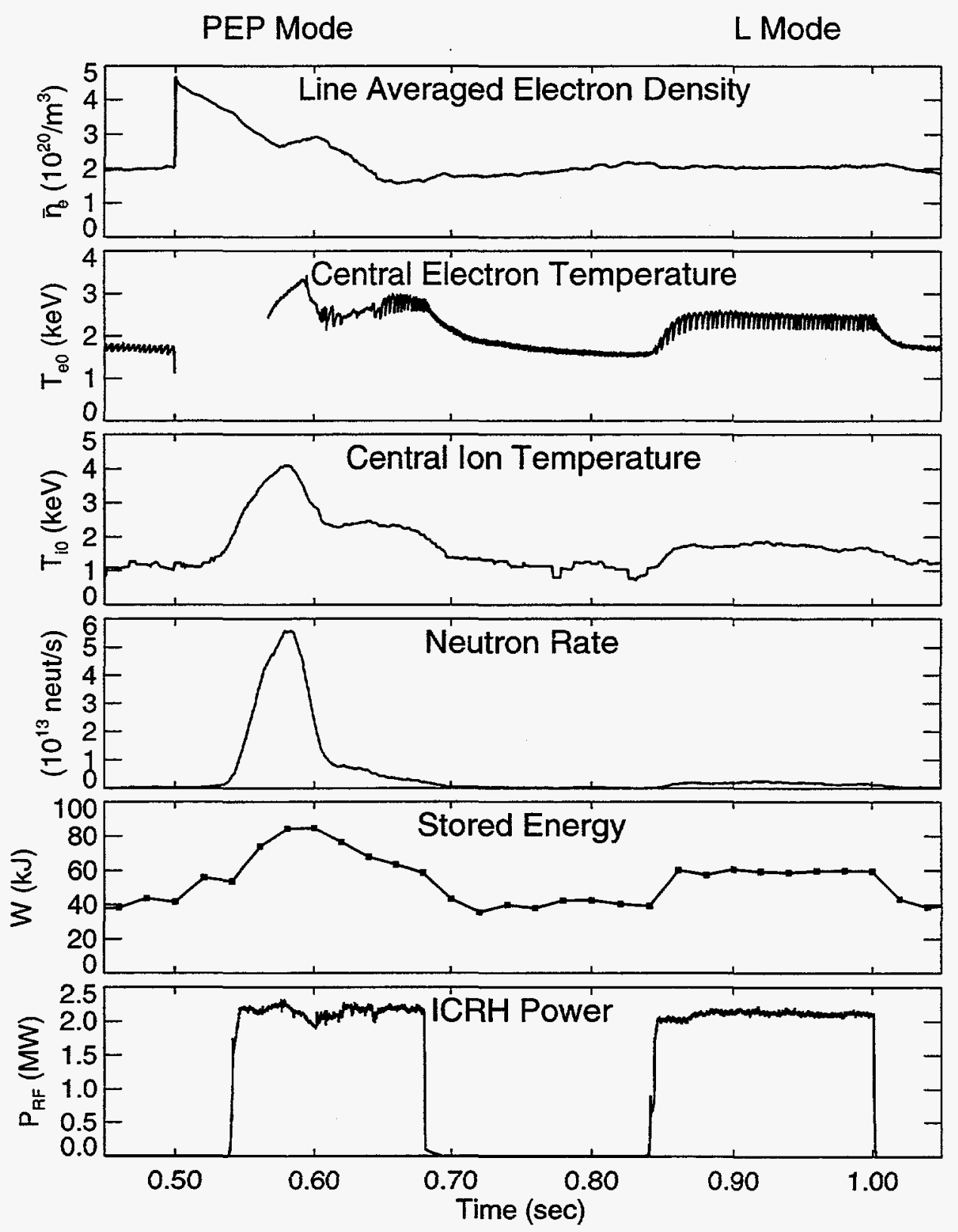

Figure 4.3: Time histories for an Alcator C-Mod discharge contrasting PEP and L-mode ICRF heated plasmas. Discharge conditions for shot 950426032: $B_{0}=5.3 \mathrm{~T}, I_{p}=1.0 \mathrm{MA}$, $\mathrm{H}$ minority ICRF heating scheme. 


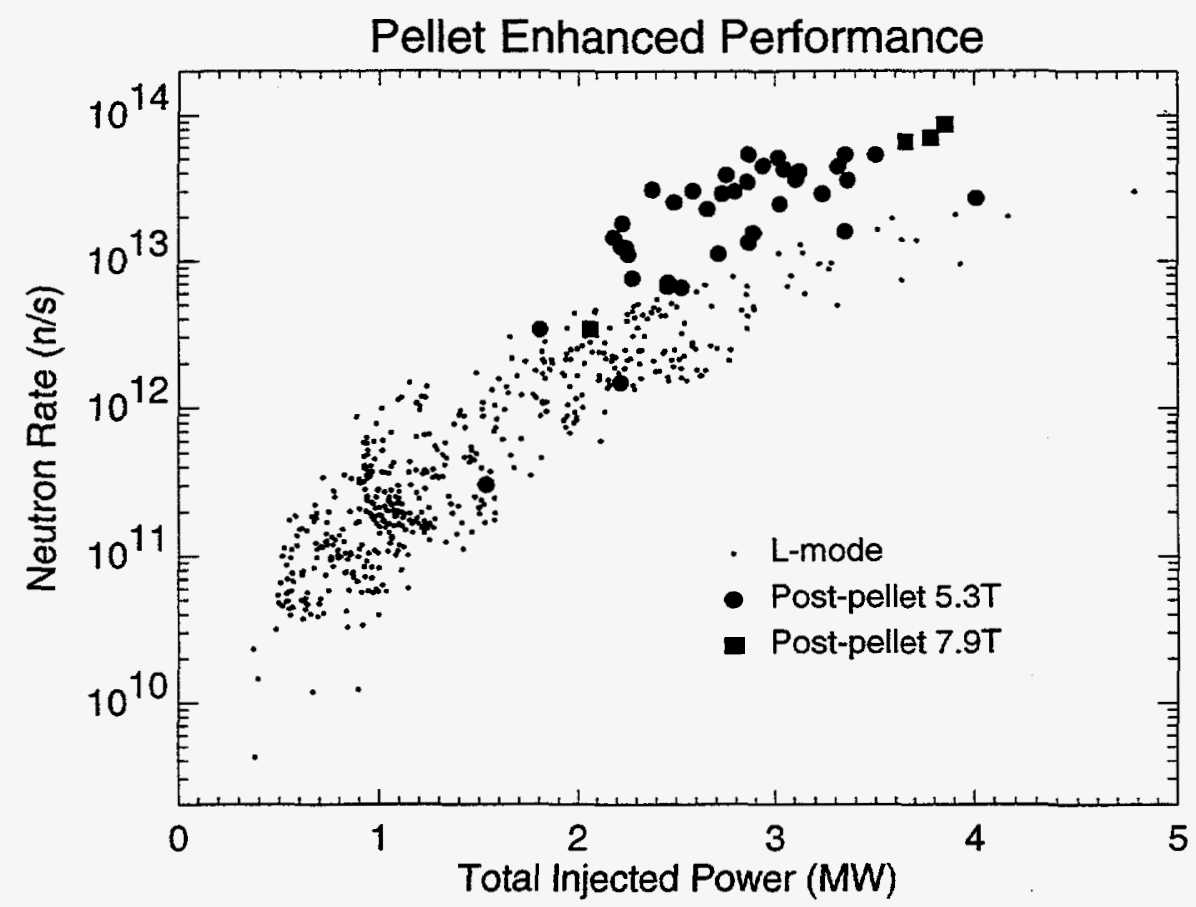

Figure 4.4: Neutron rates for a number of different Alcator C-Mod discharges plotted versus total input power. The large symbols represent post-lithium pellet discharges that did not disrupt; not all of which exhibit the characteristics of an enhanced performance discharge.

to the second ICRF pulse, no pellet has been injected and the plasma exhibits typical L-Mode behavior.

Immediately obvious is the large increase of $\mathrm{D}-\mathrm{D}$ fusion reactivity evident in the enhancement of the neutron rate by nearly a factor of 10 over the later L-mode levels. As demonstrated by Fig. 4.4, this order of magnitude increase in peak fusion performance over their L-mode counterparts is typical of the best post-pellet discharges. This enhancement in fusion reactivity is a result of the higher density at the time of the peak neutron rate accompanied by a substantial increase in the ion temperature. (In fact, the ion temperatures shown in Figs. 4.3 and 4.5 are underestimated because the assumptions made about the ion density profile used in calculating the 
ion temperature from the neutron rate are incorrect during the high density peaking of PEP modes. Comparison to the ion temperatures obtained from HIREX suggests that the neutron rate derived temperatures are underestimated by as much as $20 \%$.) During the period of highest performance of ICRF heated PEP modes, the peak ion temperature exceeds the electron temperature, contrary to the nominal L-mode behavior where electrons are preferentially heated by ohmic and ICRF power to higher temperatures.

The total stored kinetic energy of the plasma-as measured by the magneticsincreases substantially during the PEP mode and shows $\mathrm{a} \approx 30 \%$ improvement over Lmode for similar input powers. Alcator C-Mod L-mode plasmas are well described [41] by the ITER89-P [42] empirical scaling law for energy confinement time given by:

$$
\tau_{\text {ITER89-P }}=0.48 \kappa^{0.5} R^{1.2} a^{0.3} \mu^{0.5} \bar{n}_{e}^{0.1} I_{p}^{0.85} B_{T}^{0.2} P_{\text {Total }}^{-0.5}
$$

where the parameters and units are given as follows: elongation $(\kappa)$ is dimensionless, major $(R)$ and minor $(a)$ radii in $\mathrm{m}$, isotope mass number $\left(\mu \equiv m_{i} / m_{p}\right)$, average density $\left(\bar{n}_{e}\right)$ in $10^{20} \mathrm{~m}^{-3}$, plasma current $\left(I_{p}\right)$ in MA, toroidal magnetic field $\left(B_{T}\right)$ in $\mathrm{T}$, and total input power $\left(P_{\text {Total }}\right)$ in MW. Thus, the observed increase in stored energy yields a typical PEP-mode $\mathrm{H}$ factor $\left(\tau_{E} / \tau_{\text {TTER89-P }}\right)$ of 1.3 . While this is only a modest improvement of the global confinement, as we shall see in $\S 4.3 .2$, this implies a substantial improvement in the energy transport in the core of the discharge.

Unfortunately, another characteristic of the mode is its transient nature. The lifetime of the mode (as measured by the FWHM of the neutron rate enhancement peak) is typically $\approx 50 \mathrm{~ms}$ (or about two L-mode energy confinement times.) The termination of the mode is evident by a rapid drop in ion and electron temperatures (see Fig. $4.3, t \simeq .59 \mathrm{~s}$ ) 


\subsubsection{Shot 950609013}

Much of the discussion in the remainder of this chapter will focus on Shot 950609013, time histories of which are shown in Fig. 4.5. During this shot, a $B_{T}=7.9 \mathrm{~T}, I_{p}=1.0$ MA discharge with 2.5 MW of ICRF power ( $\mathrm{D}^{3} \mathrm{He}$ minority heating), an Alcator CMod record peak neutron rate of nearly $10^{14}$ neutrons per second was obtained. ${ }^{4}$ This shot will also serve as basis for a thermal transport analysis ( $\$ 4.3 .2)$ and equilibrium modelling $(\S 4.4)$ to be discussed later.

As evident in the electron temperature signal, ${ }^{5}$ sawteeth are eliminated during the PEP mode, and for sometime after the PEP mode has ended. Although sawteeth are not suppressed during all post-pellet discharges that otherwise have PEP mode characteristics, those discharges that continue to sawteeth never develop the high central pressures that lead to the order of magnitude increases in plasma reactivity over L-mode discharges shown previously in Fig. 4.4. The lack of sawteeth in PEP mode discharges indicates that the safety factor, $q$, has likely risen above 1 shortly after the injection and suggests a substantial change in the $q$ profile that will be discussed further in $\$ 4.4 .1$.

Another characteristic of auxiliary heated PEP modes is the large scale core MHD activity evident on the $\mathrm{x}$-ray and ECE signals. While this visible MHD activity is often present at the time of the PEP mode collapse, it does not appear to be driving the collapse of the enhanced confinement. This conclusion is based on two observations: some discharges show no MHD activity prior to the loss in confinement; when activity is present, it often starts well before the collapse and exhibits growth rates that do not correspond to the more rapid losses typical of the collapse. Furthermore, the activity sometimes continues well after the collapse of the PEP mode. On the other

\footnotetext{
${ }^{4}$ This record was recently broken (Feb. 1996) by high performance boronized wall H-mode discharges.

${ }^{5}$ At this higher magnetic field, the ECE electron temperature diagnostic is not adversely affected by the pellet injection.
} 


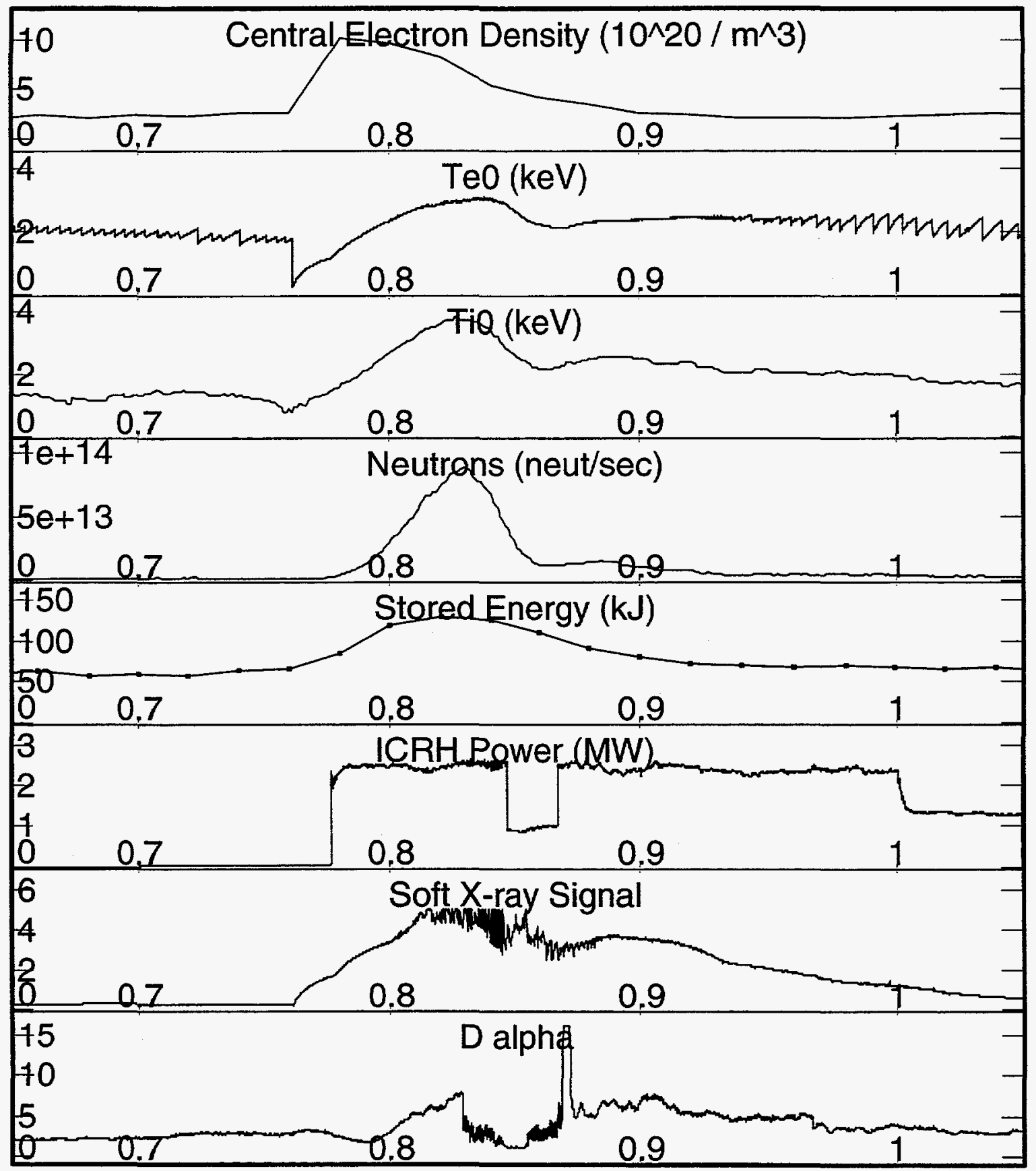

Figure 4.5: Time histories for Shot 950609013 , an $8 \mathrm{~T} \mathrm{D-}{ }^{3} \mathrm{He}$ ICRF heated PEP mode. 
hand, some kinds of MHD activity may be significant, as will be discussed in §4.4.3.

\subsubsection{Peaked profiles}

Also depicted in Fig. 4.5, the near central electron density, as measured by the Thomson scattering diagnostic, shows that inner core electron density is increased by a factor of four by the pellet injection. The radial profiles for this density step, indicating a highly peaked post-pellet density profile, were previously shown in Fig. 4.2. The high central density gradually falls off during the PEP mode, but the density profile, as indicated in Fig. 4.6 remains extremely peaked well into the PEP mode phase of the discharge.

Surprisingly, not only does the density profile become peaked by the injection of a pellet, but so do the temperature profiles, particularly the ion temperature profile. Also shown in Fig. 4.6 are the ion and electron temperature profiles for three times during the plasma shot. We see that the overall electron temperature profile is increased, with a slight increase in peakedness. However, the ion temperature becomes extremely peaked, nearly matching the gradient scale of the density profile.

This leads to perhaps the most defining characteristic of a PEP mode: large central pressures with steep internal pressure gradients. Figure 4.7 shows three total plasma pressure profiles $\left(p \equiv n_{e} T_{e}+n_{i} T_{i}\right.$ ) for the shot 950609013 . During the prepellet ohmic phase of the discharge, the plasma pressure profile is relatively flat, while during the PEP mode, the pressure is extremely peaked on axis. A large pressure gradient is observed with scale length

$$
\ell_{p}=\left(\frac{1}{p} \frac{d p}{d r}\right)^{-1}
$$

as small as a/10. Such a large pressure gradient is indicative of the creation of a transport barrier, inside of which the plasma confinement is greatly improved. Further 

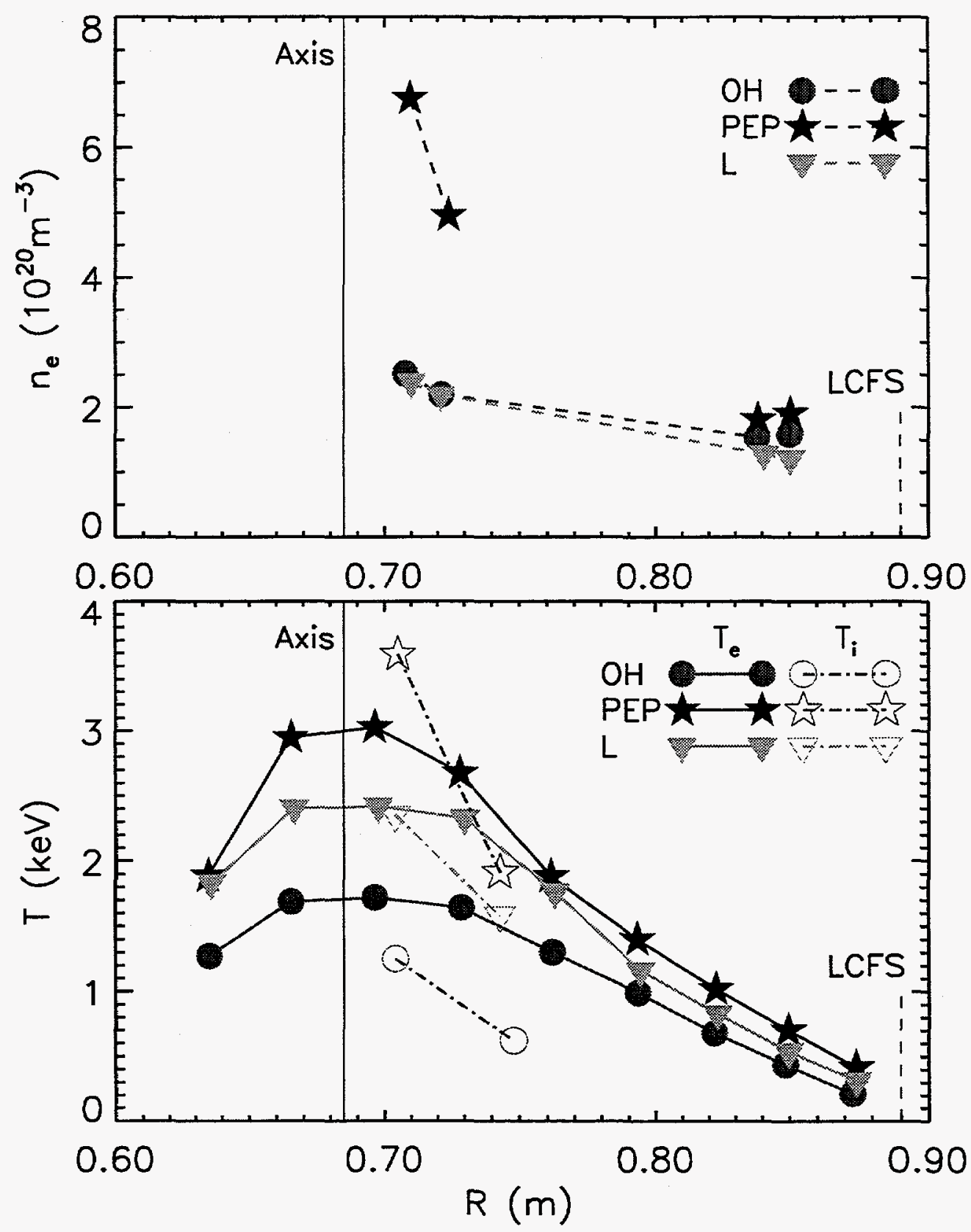

Figure 4.6: Plasma density and temperature profiles for shot 950609013 for three different times: Pre-pellet ohmic discharge $(760 \mathrm{~ms})$, PEP mode at the time of high reactivity (830 ms), and post-PEP L-mode (920 ms). 


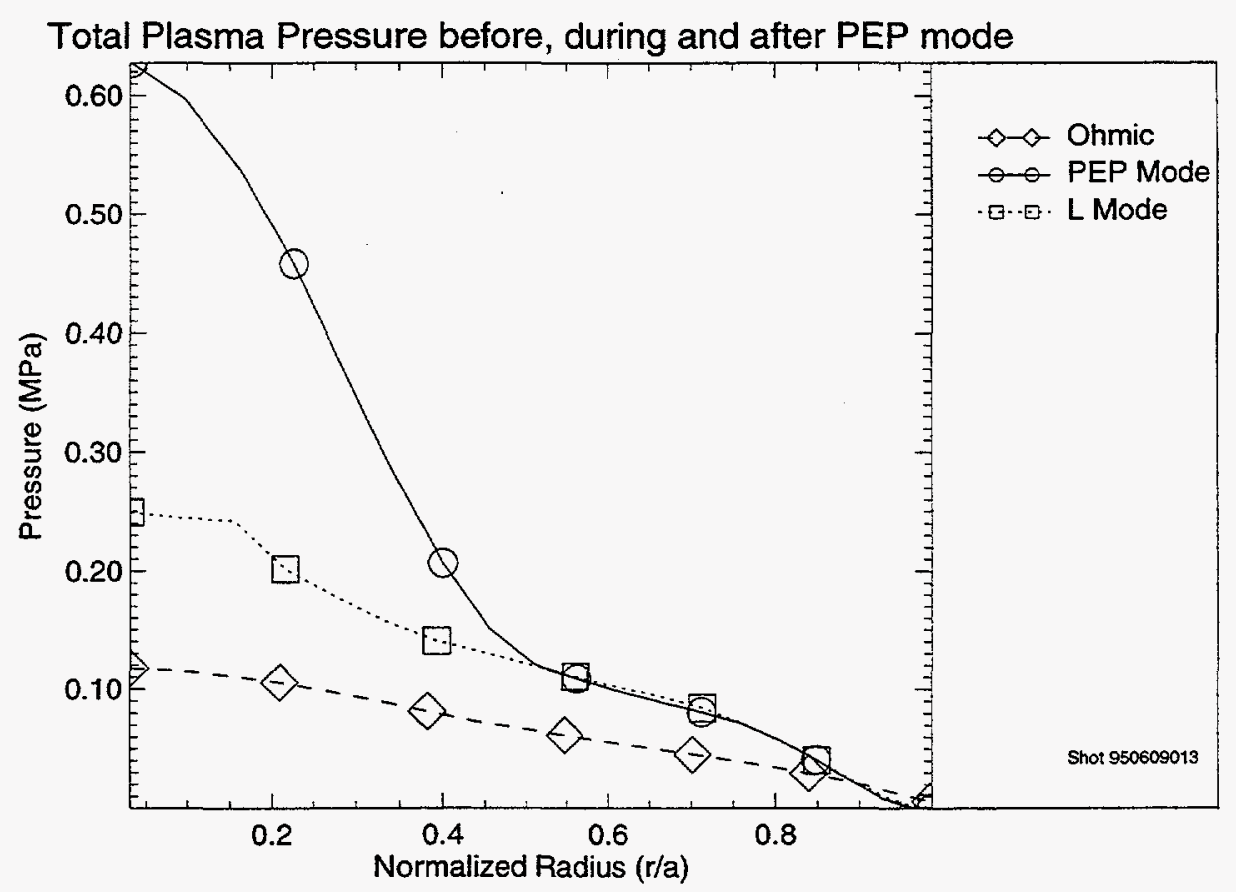

Figure 4.7: Total plasma pressure profiles for shot 950609013 .

notice that as the PEP mode collapses, the high central pressure is lost, while the outer half of the plasma remains largely unaffected.

By observing the evolution of the density profile during the PEP mode, we may track the motion of this transport barrier. A surface plot of the electron density for this discharge is shown in Fig. 4.8. After the pellet injection, the density quickly relaxes to an equilibrium profile with a fixed point of inflection near $\rho=.4$. As the central density gradually declines during the PEP mode, this point remains relatively motionless. After the central pressure collapse begins, however, this inflection point moves rapidly inward. ${ }^{6}$

\footnotetext{
${ }^{6}$ Also visible on this graph is the formation of a $\mathrm{H}$-mode edge transport barrier shoulder indicated by the elevated plateau in the outer half of the profile.
} 


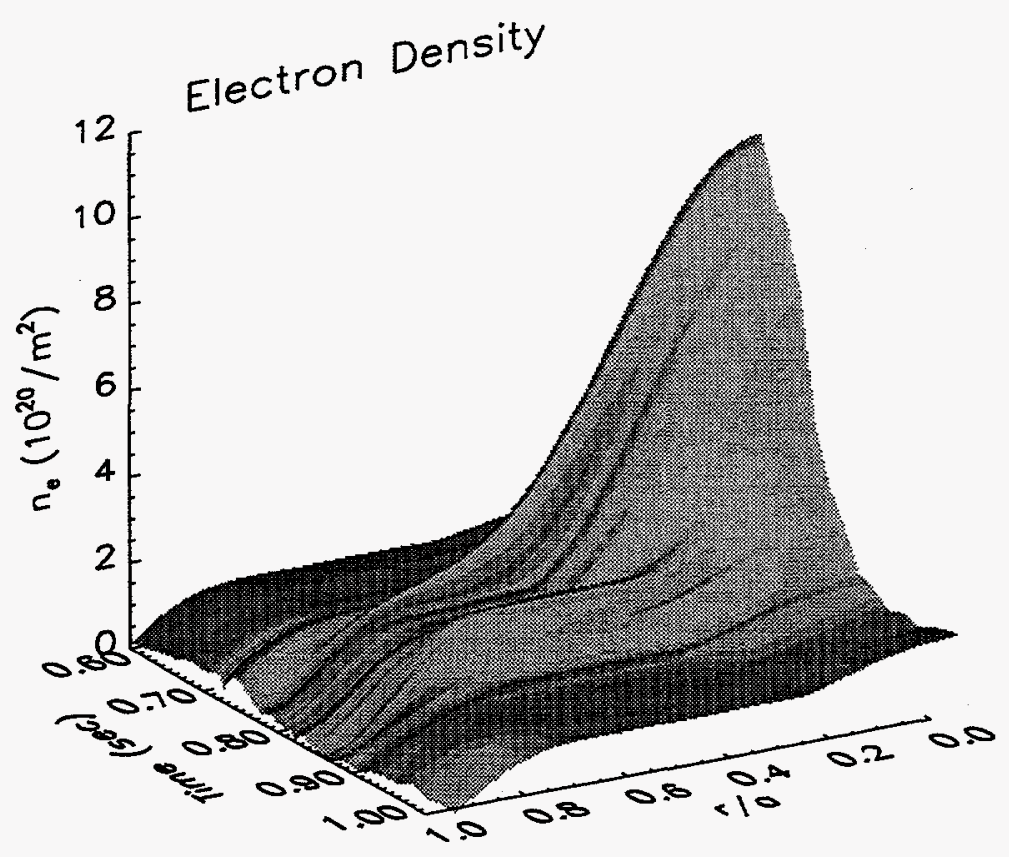

Figure 4.8: Surface plot of electron density profile as it evolves in time for shot 950609013 . The stationary nature of the transport barrier is easily seen.

\subsubsection{Other PEP mode Experimental Results}

Other phenomena of PEP mode discharges have also been discovered. For example, several experiments have been conducted to produce similar results with deuterium, rather than lithium pellet injection. However, the high fusion reactivity characterizing lithium pellet enhanced performance modes have not been seen with deuterium pellets on Alcator C-Mod. Typically, a deuterium pellet injection will begin to develop peaked profiles, but will suffer an internal collapse well before the mode has fully developed. This may be a result of the poorer penetration of deuterium pellets or a less favorable deposition profile. Alternatively, with lithium, the greater increased resistivity, due to the higher $Z_{\text {eff }}$, may evolve the discharge towards magnetic equilibria with a higher on-axis safety factor, $q$. Thus, lithium PEP mode discharges may 
possibly remain stable to the modes that are causing the early collapse of deuterium PEP modes.

With the notion that the enhancement in confinement is caused by a reversed magnetic shear configuration, as suggested by the JET results, experiments were conducted ramping the current after the injection of a pellet. It was expected that ramping the current up would increase the reversal of shear, and thus extend the length of the PEP modes. Instead, the opposite result was obtained; ramping the current up shortened the period of good confinement while ramping the current down extended it. While this result is not fully understood, the ramp-up result may be explained by the lowering of the entire $q$ profile, thus decreasing the stability of the equilibrium, as we will see in $\S 4.4 .3$.

Finally, an attempt was made to measure the poloidal rotation of the plasma. If PEP mode transport barriers can be explained by the same mechanism as $\mathrm{H}$-mode barriers, one would expect to measure a sizable radially sheared rotation speed. No rotation was observed; however, the HIREX diagnostic used for the measurement is not ideally suited to measure plasma flow and cannot resolve speeds less than $v \sim 1 \times 10^{4} \mathrm{~m} / \mathrm{s}$. The significance of this null result will be discussed in $\$ 4.6 .2$.

\subsection{PEP Mode Transport}

\subsubsection{Particle Confinement}

The formation of a steep stationary density gradient leads one to suppose that, not only is the energy confinement improved during PEP modes, but the particle confinement is improved as well. Figure 4.9 depicts a typical Ohmic PEP mode discharge-i.e. with no auxiliary heating. When the discharge drops out of PEP mode, an abrupt drop in central pressure and core impurity confinement is observed, as indicated by 

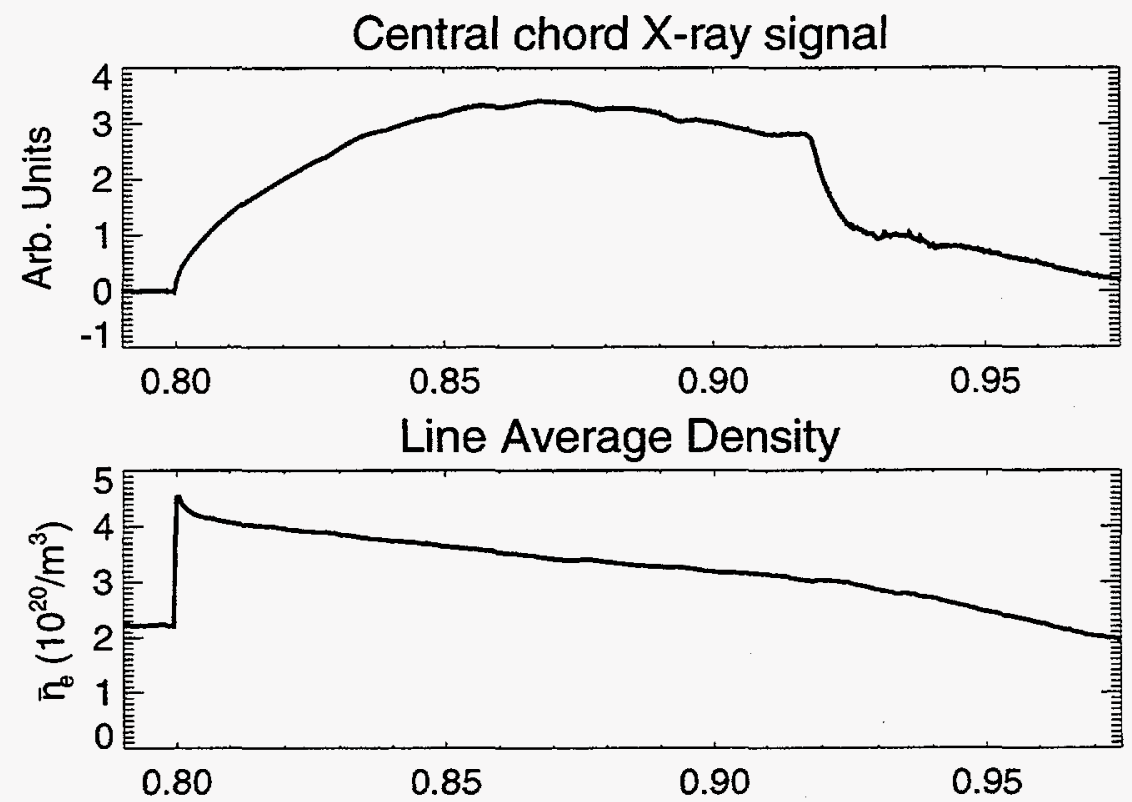

Density Decay Time
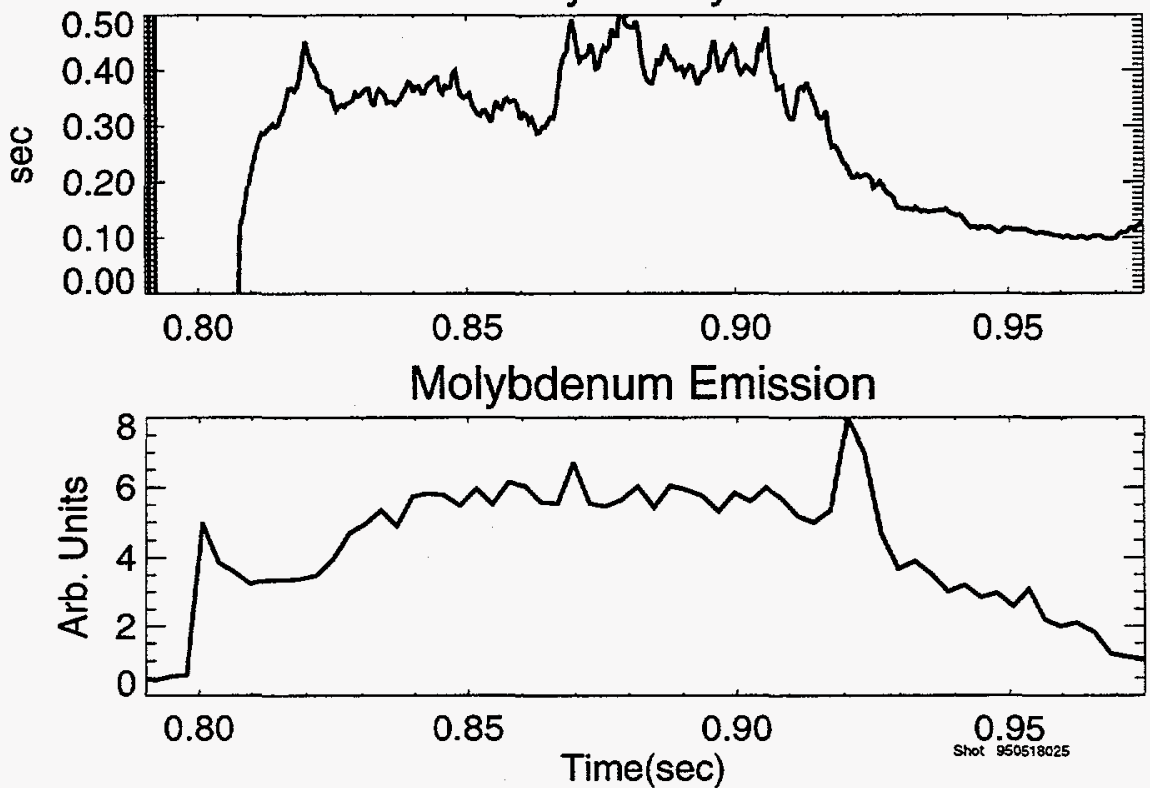

Figure 4.9: Time histories for $5.3 \mathrm{~T}, 800 \mathrm{kA}$ Ohmic PEP mode discharge, with lithium pellet injected at $0.8 \mathrm{~s}$. 
the sharp drop in central soft $\mathrm{x}$-ray emission at $0.92 \mathrm{~s}$. Coincident with the transition out of PEP mode, a break in slope is observed in the decay of central chord line averaged electron density. The average electron density decay time given by:

$$
\tau_{p}^{*}=-\frac{\mathrm{d} \ln \bar{n}_{e}}{\mathrm{~d} t}
$$

shows a improvement of 3.5-4 times that over the ohmic discharge, indicating a substantial improvement in core particle confinement. It should be noted, however, that the edge recycling source, which dominates the global particle confinement time $\tau_{p}$, is not much affected by pellet injection.

Also shown in Fig. 4.9 is the $\mathrm{Mo}^{31+}$ impurity emission signal as measured by the "Moly-monitor" [43] diagnostic. (This diagnostic measures the brightness of the plasma near the $127 \AA \mathrm{Mo}^{31+}$ line with $9 \AA$ FWHM spectral width. Thus, the diagnostic also sees the $\mathrm{Li}^{2+}$ Lyman- $\alpha$ line at $135 \AA$, evident by the sharp increase in signal when the pellet is injected. However, high resolution VUV spectroscopic measurements indicate that the lithium leaves the plasma within $50 \mathrm{~ms}$ and does not taint the subsequent signal.) The subsequent rise of the $\mathrm{Mo}^{31+}$ signal shortly after the pellet injection and decay after the central pressure collapse indicates that the Mo impurity ions accumulate in the plasma core during PEP mode.

This observation is further substantiated by an experiment designed to measure the impurity particle confinement time during PEP mode. In this experiment, a small amount of neutral $\mathrm{Ni}$ is injected by laser blowoff from a thin film of $\mathrm{Ni}$ on a glass slide into the edge of the plasma. The nickel is rapidly transported into the plasma core and then diffuses back out of the plasma at the characteristic impurity confinement time. Figure 4.10 shows the results of this experiment for two different shots. The dashed line signals show the behavior for a typical L-mode discharge. The Ni brightness rapidly increases and then decays exponentially with a decay time 

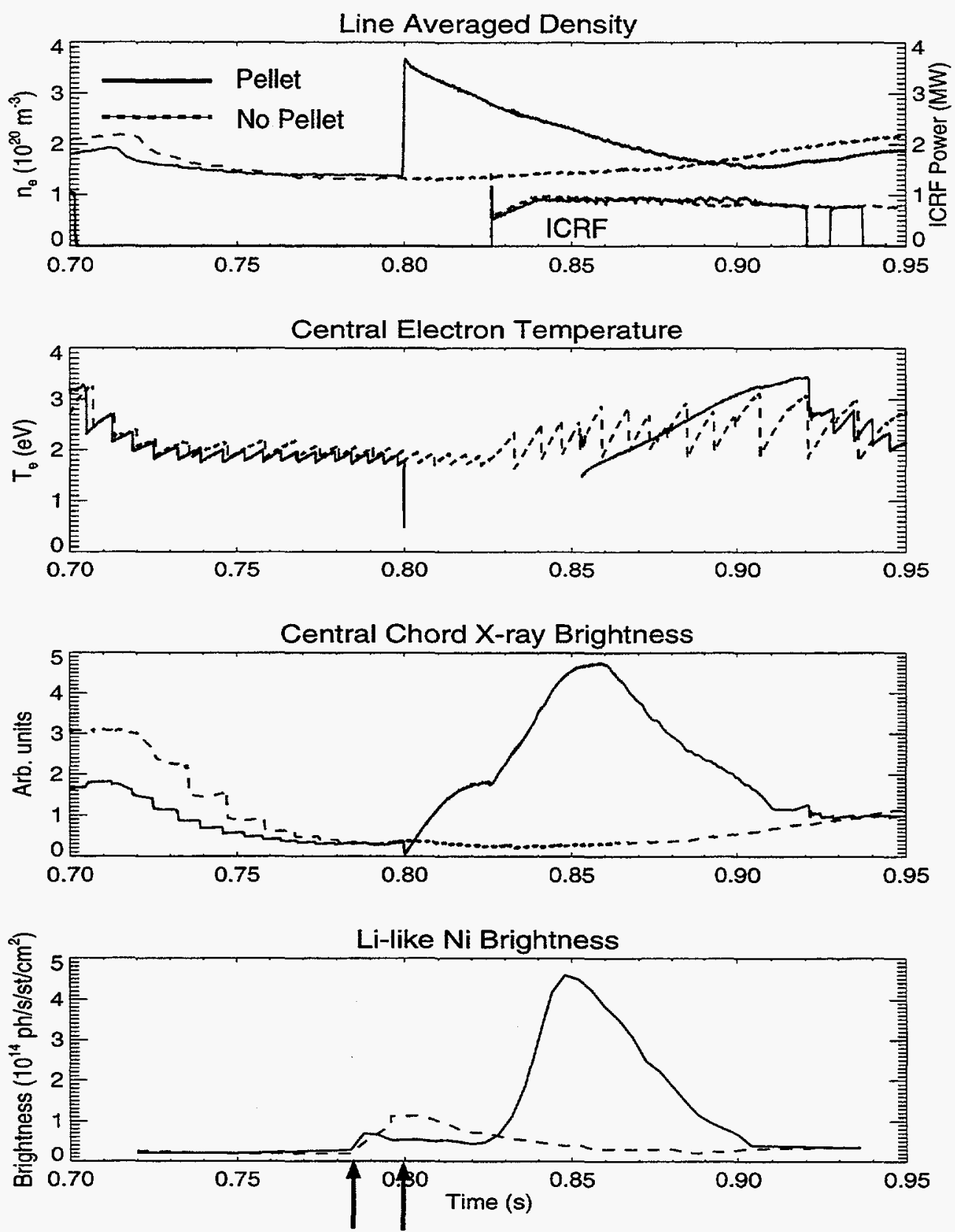

Ni Injection Pellet Injection

Figure 4.10: Time histories for two discharges with injection of Nickel at $0.79 \mathrm{sec}$. The dashed line signals are from a L-mode discharge. The solid shows a pellet injection at $0.80 \mathrm{sec}$ with a subsequent PEP mode. 
of $\approx 20 \mathrm{~ms}$. For the shot indicated by the solid lines, a lithium pellet was injected at $0.8 \mathrm{~s}, 10 \mathrm{~ms}$ after the $\mathrm{Ni}$ injection. Because the brightness of the $\mathrm{Ni}$ emission is sensitive to the changes in electron density and temperature in the post-pellet plasma, it is not possible to directly determine the particle confinement time. Notice that, after the PEP mode collapses, the $\mathrm{Ni}$ again decays away with characteristic L-mode impurity particle confinement time.

Given that there is little recycling of metal impurities injected into tokamaks, the substantially higher emission in pellet injection discharge, even after the period of enhanced confinement has ended, indicates that $\mathrm{Ni}$ is convected into the plasma core where the density and temperature are higher. The inward convection and subsequent accumulation of impurities on axis is predicted by neoclassical transport theory [44]. The observation of such accumulation indicates that the anomalous transport normally present in a tokamak discharge, which is responsible for inhibiting this accumulation, is suppressed. After the PEP mode collapses, the $\mathrm{Ni}$ again decays away with characteristic L-mode impurity particle confinement time.

A more quantitative study of impurity accumulation effect of P-mode discharges was made on originally on Alcator C. The results presented by Petrasso et al [45] indicate that impurity particle transport drops to near neoclassical levels after the injection of large deuterium pellets.

In conclusion, we've seen that PEP mode discharges have qualitatively improved core particle confinement and, furthermore, that core particle confinement is neoclassical in nature, if not in value. Better measurements of the density profiles of these discharges will allow more quantitative statements about the particle transport to be made. 


\subsubsection{Thermal Transport Analysis}

As previously mentioned, PEP mode discharges typically show a $30 \%$ improvement in global energy confinement time over L-mode scaling. However, the steep internal gradients shown by the plasma profiles indicate that the confinement improvement occurs only in the relatively small volume of the inner third of the plasma core. This suggests a more impressive improvement in the local energy confinement within the transport barrier region.

In order to quantify the local energy transport reductions, one would like to obtain values for the electron and ion thermal diffusivities, $\chi_{e}$ and $\chi_{i}$, which describe the dissipative energy transport due to neoclassical and anomalous processes. These values are derived from the dissipative heat fluxes, $\mathbf{q}_{j}=\chi_{j} n_{j} \nabla T_{j}$. To obtain the thermal diffusivities one must first solve the two-fluid energy conservation equations, given by [6]:

$$
\frac{3}{2} n_{j} \frac{d^{j} T_{j}}{d t}+p_{j} \nabla \cdot \mathbf{v}_{j}+P_{j}: \nabla \mathbf{v}_{j}=-\nabla \cdot \mathbf{q}_{j}+Q_{j k}+P_{\text {heating }}-P_{\text {loss }}
$$

where

$$
\frac{d^{j}}{d t} \equiv \frac{\partial}{\partial t}+\mathbf{v}_{j} \cdot \nabla
$$

The physical interpretation of the terms in these equations are given as follows: $\frac{3}{2} n_{j} \frac{\partial T_{j}}{\partial t}$ is the time derivative of the kinetic energy (neglecting the contribution from plasma flows). $\left(\mathbf{v}_{j} \cdot \nabla\right) T_{j}$ is the power density that heats the plasma during adiabatic compression. $p_{j} \nabla \cdot \mathbf{v}_{j}$ is power convected by particle motion. $P_{j}: \nabla \mathbf{v}_{j}$, where $P_{j}$ is the stress tensor, is the power transported perpendicular to the plasma flow as a result of plasma viscosity. $Q_{j k}$ is the power exchanged between plasma species-thus $Q_{i e}=-Q_{e i}$. $P_{\text {heating }}$ is the plasma heating source and, for ICRF heated discharges, 
is comprised of two terms: the ICRF heating power, $P_{I C R F}$ and ohmic power, $P_{O H}$. The loss power term, $P_{l o s s}$, describes the energy lost through radiation or charge exchange with neutrals (the latter term is generally very small in the core region of the plasma). Finally, $\nabla \cdot \mathrm{q}_{j}$ is the conducted density power that we wish to solve for. $\nabla \cdot \mathbf{q}_{j}$ can then be integrated over the volume contained within a flux surface to give $\mathbf{q}_{j}$ and thus $\chi_{j}$ :

$$
\begin{aligned}
\chi_{j} & =\frac{\mathbf{q}_{j}}{n_{j} \nabla T_{j}} \\
& =\frac{1}{n_{j} \nabla T_{j}} \int_{0}^{\psi} \nabla \cdot \mathbf{q}_{j} \frac{d V}{d \psi} d \psi .
\end{aligned}
$$

While the plasma density, temperature and pressure profiles are determined from diagnostic measurements, allowing the change in kinetic energy term to be determined, many of the other terms in the power balance equations are not directly observable and must be modelled from the known parameters.

The modelling of these unknowns and solving for the energy transport coefficients is accomplished using a monolithic code called TRANSP, developed at PPPL [46] by $\mathrm{D}$. McCune and co-workers and maintained for the Alcator C-Mod experiment by J. Schachter [47]. Because these energy equations involve radial and temporal derivatives, the profile data must be finely resolved. The TRANSP results presented in this section were obtained using experimental measurements of the electron density profile as determined from a combination of data from the Thomson scattering, visible bremsstrahlung, and reflectometry diagnostics and the electron temperature profile from the GPC ECE diagnostic. The ion temperature profile was obtained from two chords of the HIREX spectrometer system. $Z_{\text {eff }}$, the effective ion plasma charge, is determined from visible bremsstrahlung emission and Thomson scattering core density diagnostics and is taken to have a flat profile. By assuming a fixed particle confinement time for the injected lithium, the ion density profile can then be 
determined from $Z_{\text {eff }}$ assuming quasi-neutrality. The final important experimentally determined value is the radiated power profile, measured with a bolometer array.

The convected power is determined from particle flux, which is calculated from the density profiles assuming a fixed global particle confinement time. The contribution of viscosity, which is theoretically very small for these plasma parameters, is neglected.

The ohmic input power, $P_{O H}$, which affects only the electron power balance, is modelled assuming neoclassical resistivity and solving the current diffusion equation for the current density profile. The ICRF heating profile, $P_{I C R F}$ is modelled using the SPRUCE and FPPRF code modules assuming total absorption of the injected power $[48,49]$.

The ion-electron energy exchange power, $Q_{i e}$, is computed from classical theory. Since $Q_{i e} \propto\left(T_{e}-T_{i}\right)$, when the measured $T_{e}$ and $T_{i}$ are of the same magnitude, errors in the temperature measurements may propagate to large errors in the calculated $Q_{i e}$. Thus, under these circumstances it is difficult to separately calculate $\chi_{e}$ and $\chi_{i}$. By adding both energy conservation equations together, thereby eliminating the $Q_{j k}$ terms, one may solve for an "effective" thermal diffusivity that does not suffer from this vagary:

$$
\chi_{e f f}=\frac{\mathbf{q}_{e}+\mathbf{q}_{i}}{n_{e} \nabla T_{e}+n_{i} \nabla T_{i}} .
$$

Finally, employing the TRANSP code to solve Eqs. (4.4-4.7) for shot 950609013, we obtain the results shown in Fig. 4.11. The radial profiles of the ion, electron, and effective thermal diffusivities, $\chi_{i}, \chi_{e}, \chi_{e f f}$ respectively, are calculated for three times during the discharge: during the pre-pellet ohmic stage, during the PEP mode near the time of the peak neutron rate, and following the collapse central pressure when the plasma has returned to L-mode confinement. The measured diffusivities are good near the core of the discharge wher the ion temperature profile data is valid. The 
Thermal Diffusivites for Shot 950609013
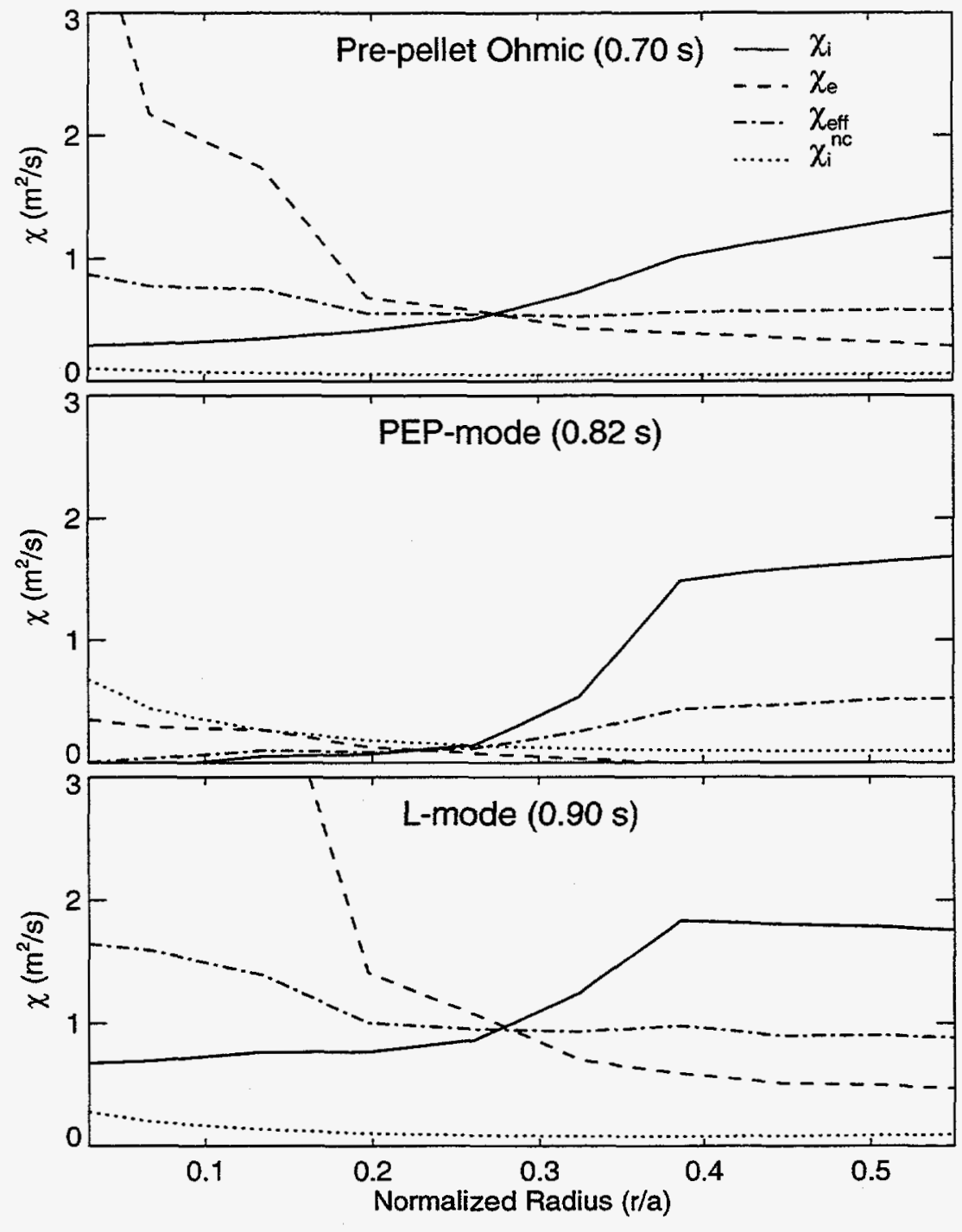

Figure 4.11: Ion, electron and effective thermal diffusivities, as inferred by TRANSP, for three times during Shot 950609013. Shown for comparison is the calculated neoclassical ion diffusivity. 
reduction of the transport coefficients during the PEP mode is readily apparant.

The core ion thermal diffusivity is reduced during the PEP mode to near the calculated neoclassical level. While this TRANSP derivation implies that the ion thermal transport is below the neoclassical value, the errors in the modelling are potentially large. Furthermore the neoclassical $\chi_{i}$ computed by TRANSP (ChangHinton [50] model) may be an overestimate because at these high ion temperatures and steep density and temperature gradients the assumed neoclassical scaling of $\ell_{p} \gg$ $\rho_{P i}$, the ion poloidal gyroradius, is not strictly met [51]. (The ion poloidal gyroradius, given by $\rho_{P_{i}} \equiv\left(\frac{B_{p}}{B}\right) \rho_{L i} \approx a / 24$ for ions at $\rho=.25$ for shot 950609013 , where $\ell_{p} \approx a / 10$.)

$\chi_{e}$ also appears to drop during the PEP mode, although not as dramatically as $\chi_{i}$. The steepening of the electron temperature profile is modest in comparison to the ion temperature profile effect (see Fig. 4.6), and as we shall see, most of the energy lost from the core is through the electron channel. Both these effects indicate that the electron thermal confinement enhancement is small compared to that of the ions. As an aside, comparing the electron thermal diffusivity of the pre-pellet ohmic discharge with the post-PEP auxiliary heated L-mode discharge reveals the usual L-mode degradation in confinement with increased input power.

The details of the radial power balance further illustrate the good ion thermal confinement during PEP mode. As shown in Fig. 4.12, the ion power balance indicates a substantial reduction in the ion conducted power, $Q_{i}=\nabla \cdot \mathbf{q}_{i}{ }^{7}$ This corresponds to the low values for $\chi_{i}$ seen in the previous graph. The high observed ion temperature of the PEP mode discharge is a consequence of the combination of improved ion thermal confinement and better coupling of the ICRF heating power to the ions. As the ion temperature increases, the main loss channel for the ion energy becomes collisional

\footnotetext{
${ }^{7}$ However, the positive value of $Q_{i}$ in the center of the discharge, which would indicate a negative value for $\chi_{i}$, is incorrect. This error is most likely caused by miscalculation of the ICRF heating power on axis.
} 


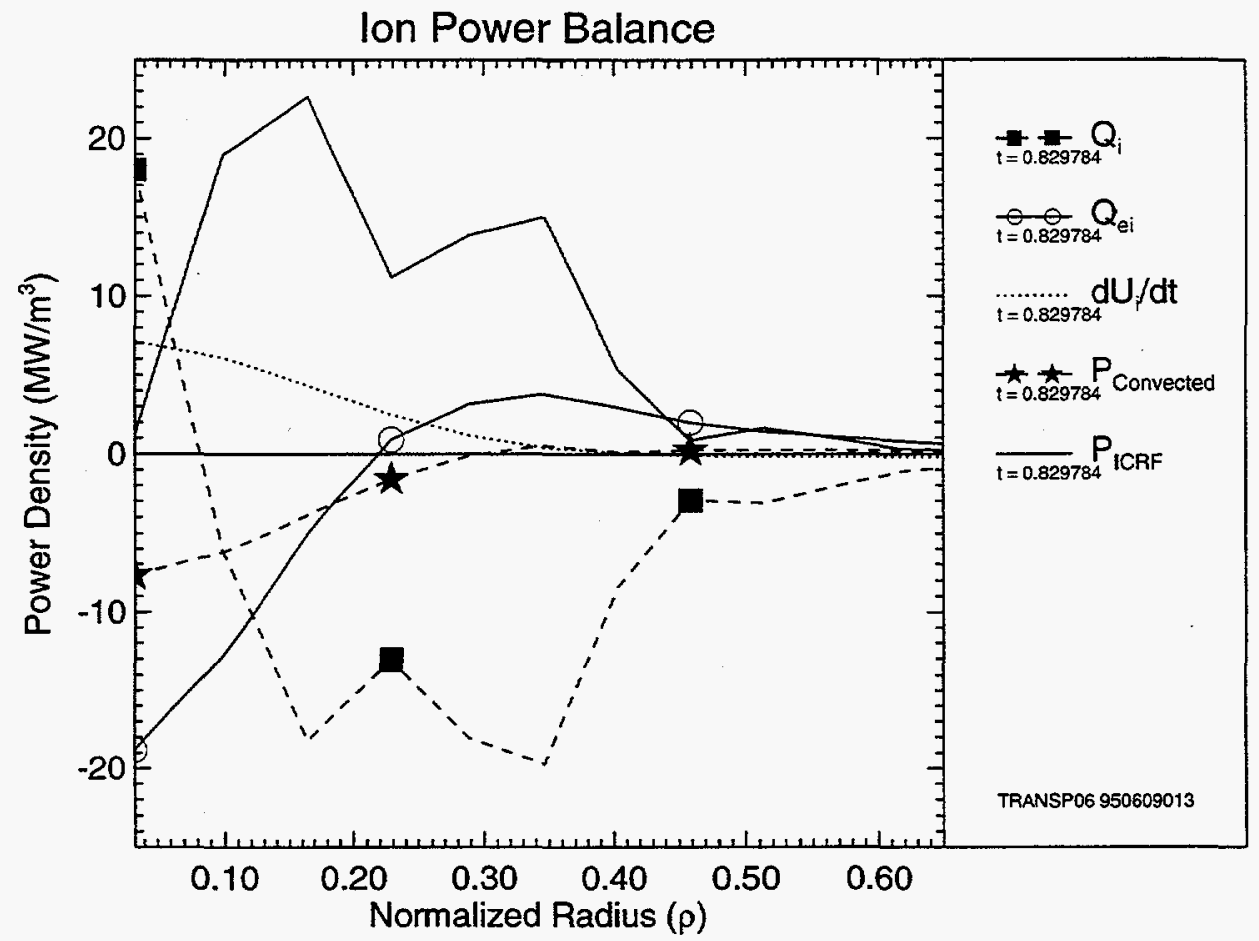

Electron Power Balance

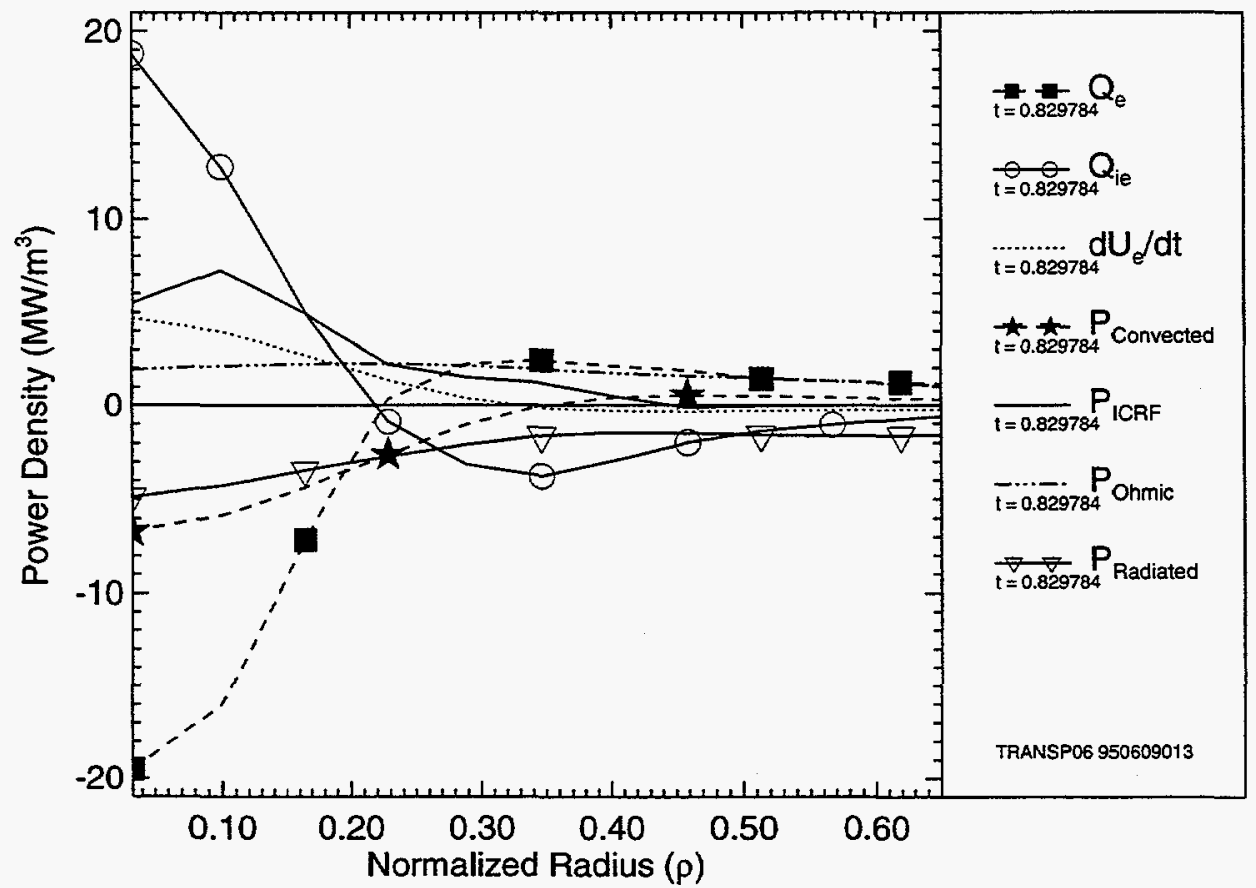

Figure 4.12: Radial power balance profiles for shot 950609013 at 830 ms. 
heat transfer to the relatively colder electrons. The core energy is then conducted out of the core by the electrons as shown in the lower plot. Again this indicates that, while the ion thermal confinement is well enhanced during the PEP mode, electron thermal transport is not similarly influenced.

\subsection{PEP mode Equilibria}

The observation that sawteeth are suppressed during PEP modes suggests that significant changes in the plasma equilibrium occur as a result of the pellet injection. The experiments at JET [39], in which the $q$ profile is inferred from the mode structure of intrinsic MHD oscillations, suggest that during the time of enhanced performance, the plasma equilibrium contains a central region with negative or reversed magnetic shear. The magnetic shear, $\hat{s} \equiv d \ln q / d \ln r$, is the radial logarithmic derivative of the $q$ profile and is ordinarily non-negative everywhere in the plasma. A region of reversed shear generally indicates a current density profile that is hollow. As we shall see in $\S 4.6 .2$, a reversed shear profile also has an important consequence for confinement, as negative shear can reduce the theoretical growth rates of turbulent modes believed to be responsible for anomalous transport.

\subsubsection{LPI $q$ profile measurements}

As described in Chapter 3, the Alcator C-Mod Lithium Pellet Injection diagnostic system is capable of measuring the plasma $q$ profile. In this section, we will discuss the results of a measurement of the $q$ profile for a PEP mode discharge.

The basic experiment, shown in Fig. 4.13, involves the injection of a lithium pellet into a $5.3 \mathrm{~T}, 800 \mathrm{kA}$ discharge. The injection is followed shortly by the turning on of $1 \mathrm{MW}$ of ICRF heating power to achieve a high performance PEP mode. When the PEP mode is fully developed, a second pellet is injected to diagnose the current 


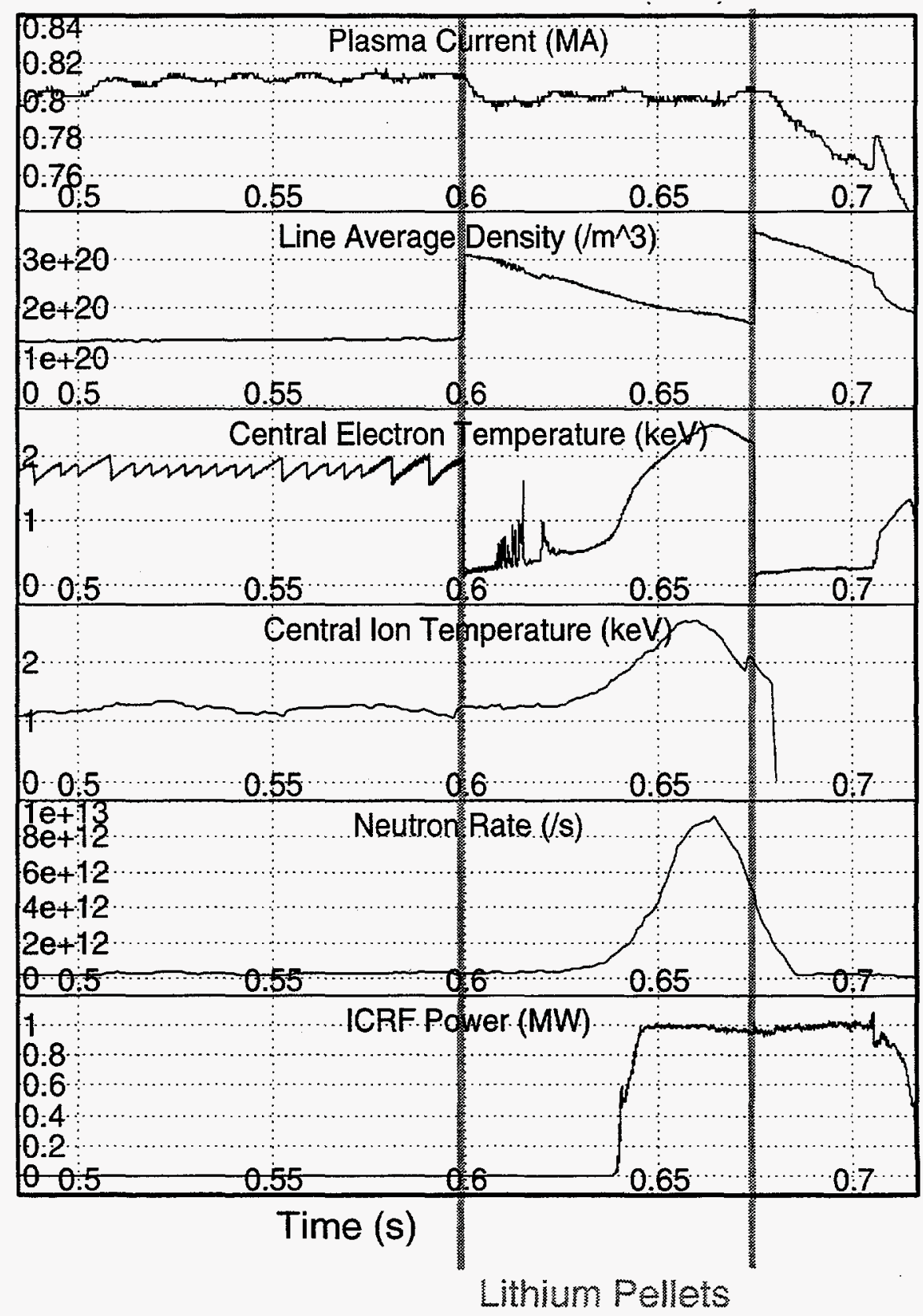

Figure 4.13: Time histories from double pellet injection experiment used to measure the current density profile during PEP mode. The first pellet is injected at $0.6 \mathrm{~s}$ to produce the PEP mode, while the second pellet injected at 0.675 measures the current density profile. 
density profile.

The 'Cigar Detector' technique was used to measure the magnetic filed tilt angle from an image of the ablating pellet. This technique, as well as the particular details of the measurements for this experiment, were described in $\S 3.4$. Because the cigar detector diagnostic is only capable of taking a single image per injected pellet, and thus a single local measurement of the magnetic field tilt angle, it is necessary to repeat the entire shot multiple times, varying the camera snapshot time, to obtain the radial profile.

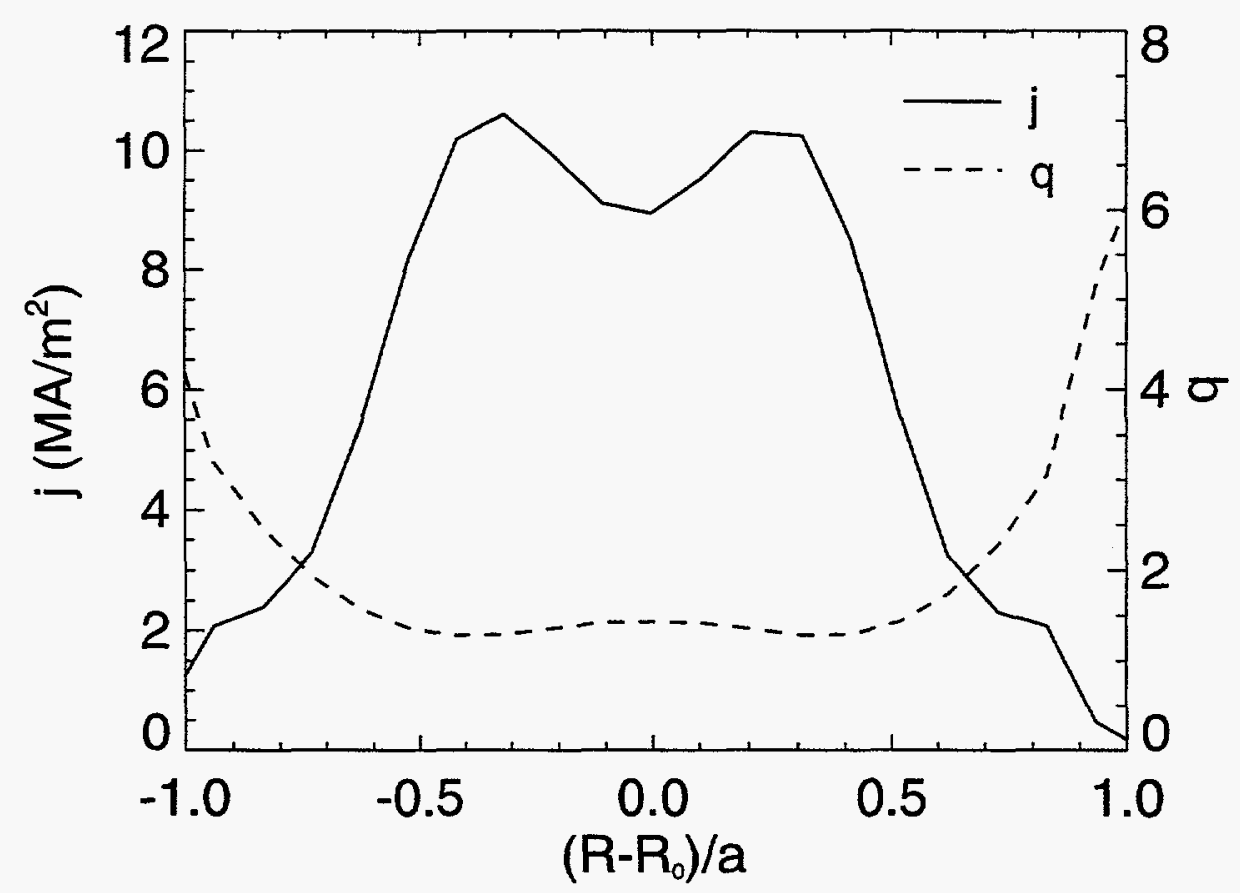

Figure 4.14: Current density and $q$ profiles from EFIT equilibrium fit to 'Cigar Detector' measurements of the internal magnetic field tilt angle from repetitive shot PEP mode experiment. A hollow current profile and central region of weakly negative shear is clearly evident.

The results of this series of shots are shown in Fig. 4.14. The data indicate a 
region of reverse shear exists in the core of the plasma, in agreement with the JET result.

\subsubsection{TRANSP $q$ profile modelling}

It is perhaps a surprising result that the current density profile can be so dramatically affected by the injection of a pellet: from a highly peaked profile to a nominally hollow one. The JET team speculated that the current profile hollowness is the result of a significant amount of bootstrap current being driven off-axis by the steep pressure gradient. However, given the very high densities of post-pellet Alcator C-Mod plasmas, one might expect very little bootstrap current, a consequence of neoclassical transport in the low collisionality (and low density) "banana" regime.

Along with the ability to solve for the transport coefficients, discussed in $\$ 4.3 .2$, TRANSP also contains an equilibrium solving code, VMEC [52], that can solve for the current density profile using the time dependent neoclassical poloidal magnetic field diffusion equation [53]. Furthermore, TRANSP can also model the neoclassically predicted bootstrap current. The results of the TRANSP modelling of the current density profiles for the 7.9T PEP mode discharge is shown in Fig. 4.15. The bootstrap current due to the large pressure gradient at the transport barrier (as previously shown in Fig. 4.7) is roughly $30 \%$ of the local current density. This results in a slightly hollow overall current density. Thus, these TRANSP results are consistent with the notion that the observed region of reversed shear is due to the formation of bootstrap current. ${ }^{8}$

Figure 4.16 depicts the time histories of TRANSP outputs at $\rho=.2$, near where the bootstrap current density peaks. This figure illustrates the earlier concern that the

\footnotetext{
${ }^{8}$ However, a caveat must be made as the TRANSP calculation of the bootstrap current does not include the full neoclassical calculation and specifically makes approximations that may not be valid for the high collisionality observed immediately after the pellet injection. It is not clear what impact the inclusion of the full calculation will have on these results.
} 


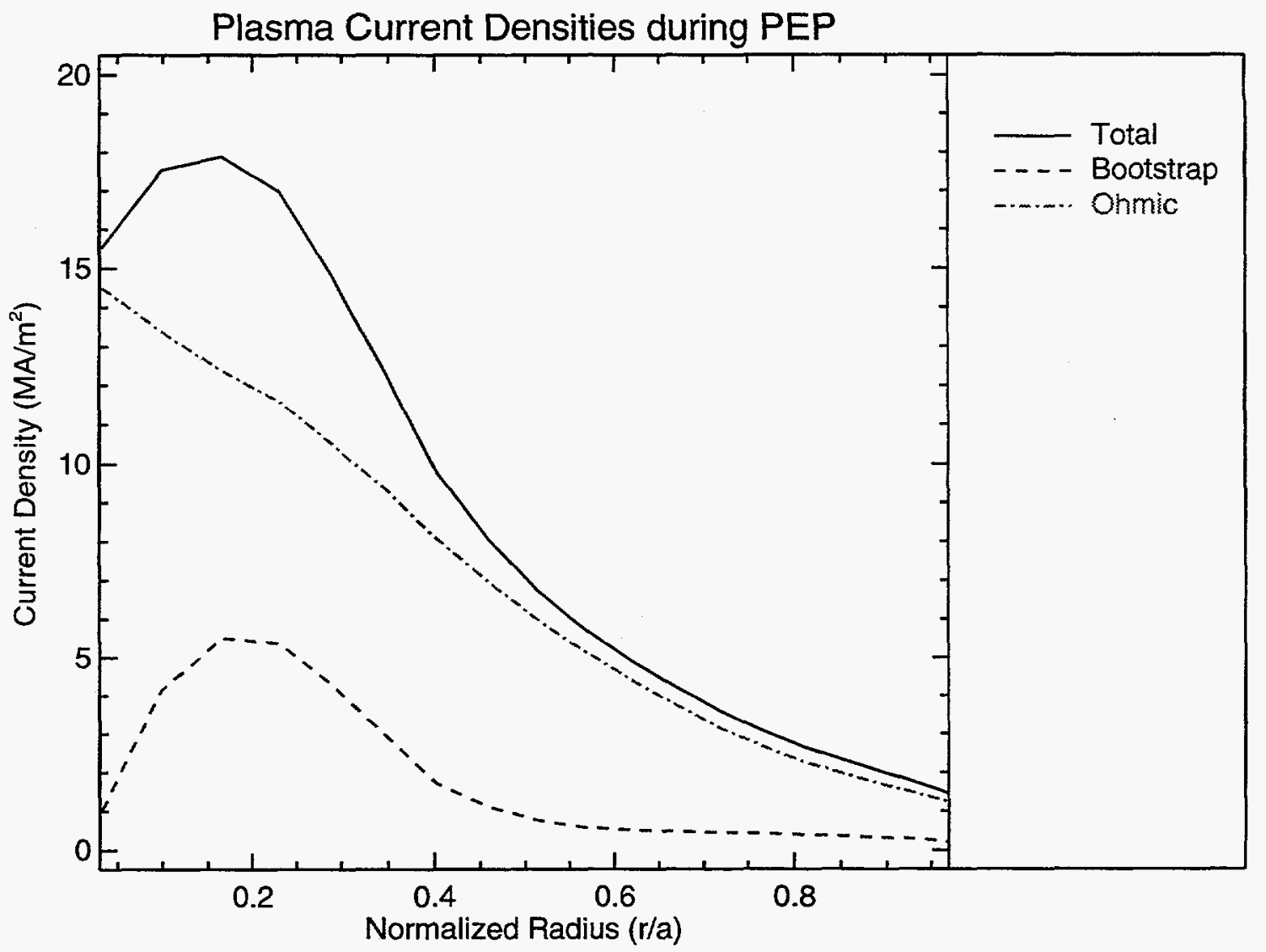

Figure 4.15: Current density profiles from TRANSP poloidal field diffusion equilibrium modelling for Shot 950609013 during the PEP mode at $820 \mathrm{~ms}$, just before the peak of neutron rate.

collisionality of these discharges are too high after the pellet injection for formation of bootstrap current. The collisionality parameter

$$
\nu_{e}^{*}=\frac{\nu_{c}}{\omega_{b}}
$$

is the electron Coulomb collision frequency normalized to the bounce frequency of the trapped electrons that travel in 'banana orbits'. The transition from the low to intermediate collisionality regimes, the banana-plateau transition, nominally occurs 
Plasma Parameters at $\rho=.2$
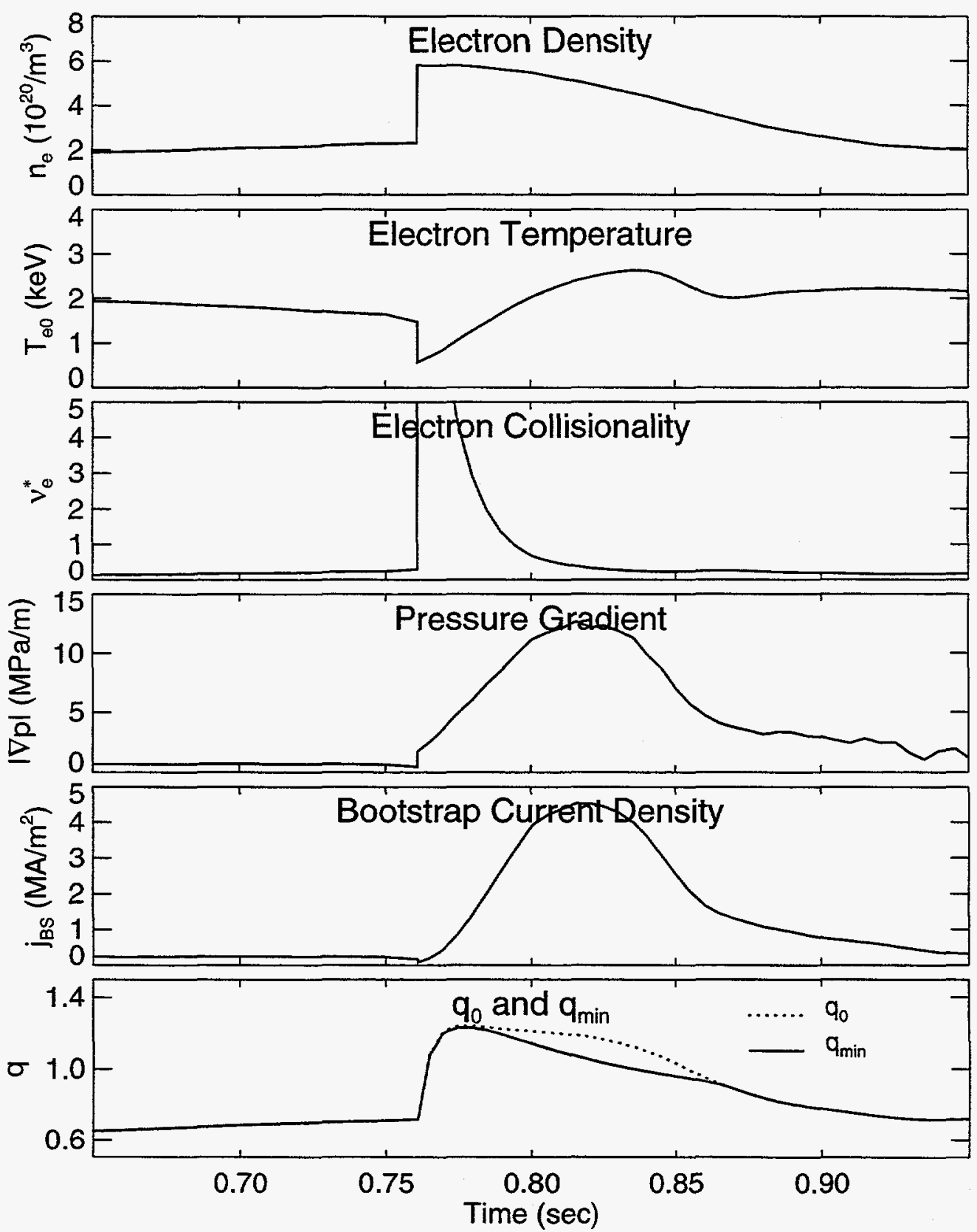

Figure 4.16: Plasma parameters used or calculated by TRANSP to model the bootstrap current at $\rho=.2$. Also shown is $q_{0}$ and $q_{\min }$ as a function of time; when they deviate, a region of negative shear exists in the plasma core. 
for $\nu_{e}^{*} \sim 1$ [8]. Although $\nu_{e}^{*}$ is initially very high after the pellet injection, it rapidly drops below 1 as the plasma reheats. Combined with the large pressure gradient, this leads to the substantial bootstrap current and the formation of a central region of reversed magnetic shear.

The existence of the reversed shear is indicated in this figure by the deviation of $q_{\min }$ from $q_{0}$. The time histories of these parameters suggest that the region of reversed shear persists after the loss in central energy confinement enhancement, as indicated by the rapid drop in electron temperature. This suggests that reversed magnetic shear is not a sufficient condition for the enhanced confinement behavior. This suggestion by the TRANSP modelling analysis is substantiated by the experimental measurement. A careful look back at the $q$ profile measurement experiment (recall Fig. 4.13) reveals that the experimental measurement of the reversed shear profile occurred $10 \mathrm{~ms}$ after the peak in the neutron rate, during the collapse of the PEP mode. Thus, the reversed shear region exists after the end of the enhanced confinement, confirming that a reversed shear equilibrium is not a sufficient condition for the enhancement of confinement.

Furthermore, this figure demonstrates that the bootstrap current and reversed shear region take some time to develop after the injection of the pellet. Based on the delay in its onset, one may argue that reversed shear is also not a necessary condition for enhanced confinement; rather, it is a consequence of the pressure gradient that builds up because of the enhanced confinement. However, because the beginning of enhanced confinement is not well defined, this argument is not justified. In some cases, the core density is observed to rapidly drop up to $\approx 10 \mathrm{~ms}$ after the injection of the pellet, then increase as the confinement improves, and finally begin to decay away at a slower rate. This suggests that the transition to enhanced confinement may also be delayed some time after the pellet penetration to the core. So, inconclusively, reversed shear may exist during all of the enhanced confinement period. The issue of 
whether reversed magnetic shear is a necessary condition for enhanced confinement will be revisited in $\S 4.6 .2$.

\section{Ramifications of Reversed Shear Equilibria}

Operationally, one difficulty of the PEP mode equilibrium is that the temperature and current density profiles are not 'aligned'. Because the plasma resistivity is a strong function of the temperature, the inductively driven portion of the plasma current will diffuse towards the region of high temperature and low resistivity. As the temperature increases during PEP mode, the overall current density profile will become more peaked, lowering $q_{0}$ and the inner $q$ profile until $q_{0}$ or $q_{\text {min }}$ drops below 1 , at which point the discharge will resume sawtoothing. While the low resistivity of these discharges will serve to retard this inward diffusion of current, the PEP mode discharge is inherently not steady-state.

\subsubsection{Ideal MHD Stability Analysis}

While some PEP modes clearly terminate by the return of sawteeth, in others the onset of sawteeth are delayed up to $100 \mathrm{~ms}$ after the end of the enhanced confinement (e.g. shot 950609013 as shown in Fig. 4.5.) Furthermore, as discussed in the previous section, the existence of a region of reversed shear is not exactly correlated with the improvement in confinement. Obviously, a better understanding of the termination of the enhanced confinement mode is desired. A natural starting point for this investigation is the analysis of the ideal MHD stability of these discharges. Such investigation is further prompted by the observation of large coherent fluctuations on the central chord soft $x$-ray and temperature signals mentioned earlier in $§ 4.2 .1$. This section will describe the results of such an analysis.

To explore the ideal stability of PEP modes, the equilibrium of shot 950609013 


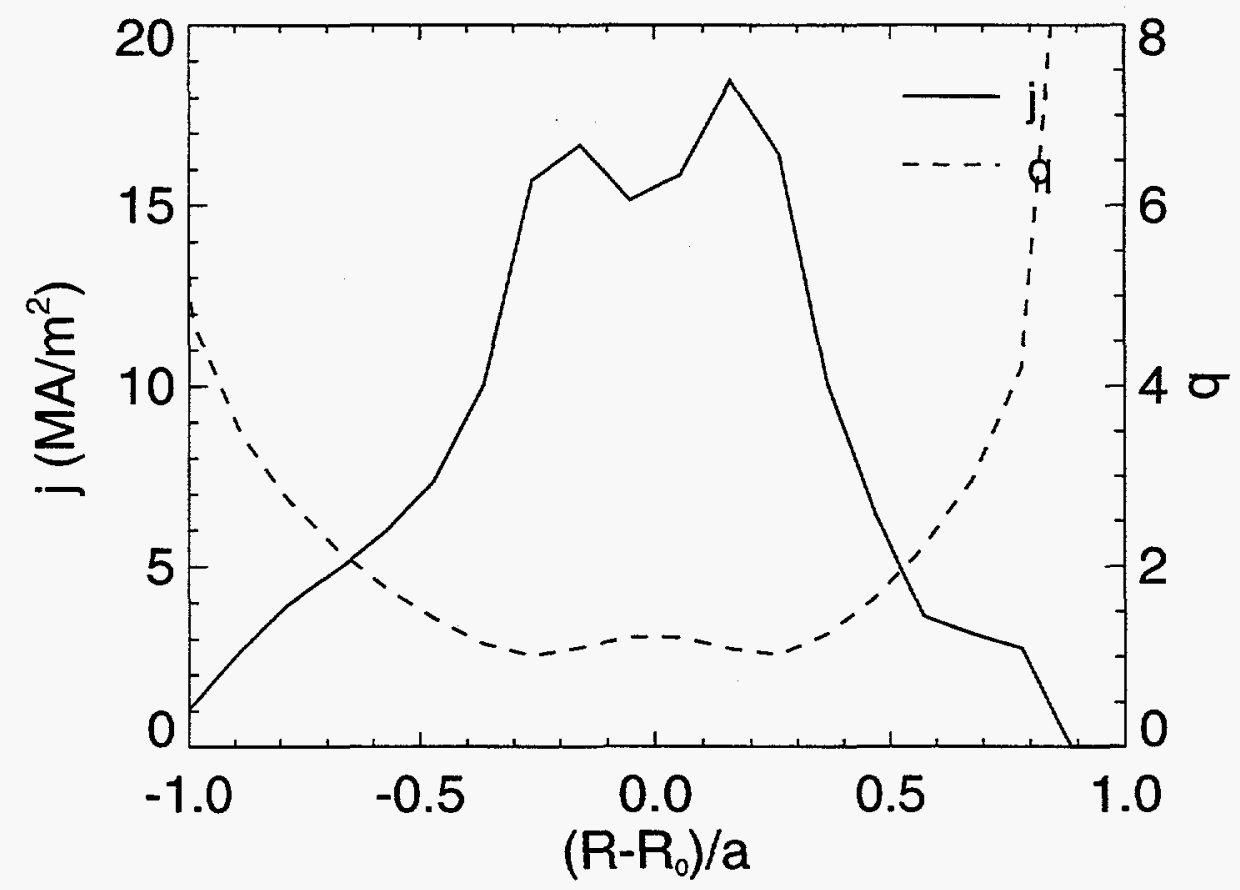

Figure 4.17: Current density and $q$ profiles from TRANSP modelling of shot 950609013 for $t=820 \mathrm{~ms}$. This equilibrium was used as a basis for the ideal MHD stability analysis.

near the time of the peak neutron rate, as modelled by TRANSP, was taken as representative of the best PEP mode discharges. The current density and $q$ profiles for this discharge are shown in Fig. 4.17. The analysis of this plasma was performed by J. Ramos using the CAXE \& KINX [54] set of codes for equilibrium construction and ideal MHD stability. The discharge was found to be stable, but as we shall see, near to the marginal stability limits for the $n=1$ kink and $n=\infty$ ideal ballooning modes.

The next step is to explore the stability of equilibria that are similar to that of shot 950609013. Recall the Grad-Shafranov equation that governs the MHD equilibrium 
of an axisymmetric toroidal plasma [6]:

$$
\Delta^{*} \psi=-F \frac{\partial F}{\partial \psi}-\mu_{0} R^{2} \frac{\partial p}{\partial \psi}
$$

where the left hand of the equation is related to the toroidal current density:

$$
\begin{aligned}
\Delta^{*} \psi & =R^{2} \nabla \cdot\left(\frac{\nabla \psi}{R^{2}}\right) \\
& =-\mu_{0} R \cdot j_{\phi}
\end{aligned}
$$

and the toroidal magnetic field is related to $F$ by

$$
F=R B_{\phi} \quad \text {. }
$$

To explore discharges with similar current density profiles, we aspire to keep fixed the left hand side of Eq. (4.9), which governs the position of the surfaces of constant poloidal flux, while varying other characteristics of the equilibrium. One possible variation would be to add an arbitrary constant to $F^{2}$. Since only the derivative of this quantity is included in the Grad-Shafranov equation, adding a constant has no effect on the plasma equilibrium shape. However, since $B_{\phi}$ is proportional to $F$, adding this constant allows us to arbitrarily scale $q^{*}$, which is proportional to the toroidal magnetic field.

$$
q^{*} \text { scaling }\left\{\begin{array}{l}
F^{2} \longrightarrow F^{2}+C \\
\frac{\partial p}{\partial \psi} \longrightarrow \frac{\partial p}{\partial \psi}
\end{array}\right.
$$

A second transformation of interest would be to scale the pressure profile. By scaling the pressure and adjusting the toroidal field for the diamagnetic change we 
can obtain nearly the same poloidal flux shape. Thus, we effectively scale $\beta_{p}$ :

$$
\beta_{p} \text { scaling }\left\{\begin{aligned}
\frac{\partial p}{\partial \psi} & \longrightarrow \lambda \frac{\partial p}{\partial \psi} \\
F \frac{\partial F}{\partial \psi} & \longrightarrow F \frac{\partial F}{\partial \psi}+\mu_{0} R_{c}^{2}(1-\lambda) \frac{\partial p}{\partial \psi}
\end{aligned}\right.
$$

However, this mapping does not give exactly the same current density profile shape because a constant 'average' major radius, $R_{c}$, is used. This cannot be helped as physically increasing $\beta_{p}$ will cause an increase in the Shafranov shift of flux surfaces.

Using the $q^{*}$ and $\beta_{p}$ scalings just described, we define a plane in "equilibrium space" of equilibria with current density profiles similar to our basis discharge. Shown in Fig. 4.18 are the results from the stability analysis of numerous equilibria on this plane. The results indicate that the basis equilibrium taken from the 7.9T PEP mode discharge is very near to both the $n=1$ and $n=\infty$ marginal stability limits, shown with heavy lines. Thus, a modest increase in the plasma $\beta$ or a slight drop in $q_{\min }$ will drive the discharge unstable. These results further indicate that a $\beta$ limit exists for the $n=\infty$ modes for $\beta_{N} \gtrsim 1.7$; discharges with higher normalized $\beta$ will be unstable to the high- $n$ ballooning modes for values of $q_{\min }$ up to 1.5. Discharges with $q_{\min }>2$ and small or negative shear enter the region of "second stability" and are stable to high- $n$ ballooning modes even with large values of $\beta$ [55].

Recall the coherent fluctuation activity of shot 950609013 shown in Fig. 4.5, which exhibited large fluctuations on the central chord $\mathrm{x}$-ray signals during most of the period of enhanced performance. While these oscillations do not appear to be causing a degradation in confinement, an examination of the GPC ECE electron temperature diagnostic signals reveal a coherent mode that is coincident with the collapse of the enhanced confinement at $840 \mathrm{~ms}$. Figure 4.19 shows a contour plot of electron temperature radial profile as a function of time with an unambiguous coherent oscillation. Notice that as the oscillation grows, the central temperature is falling while 


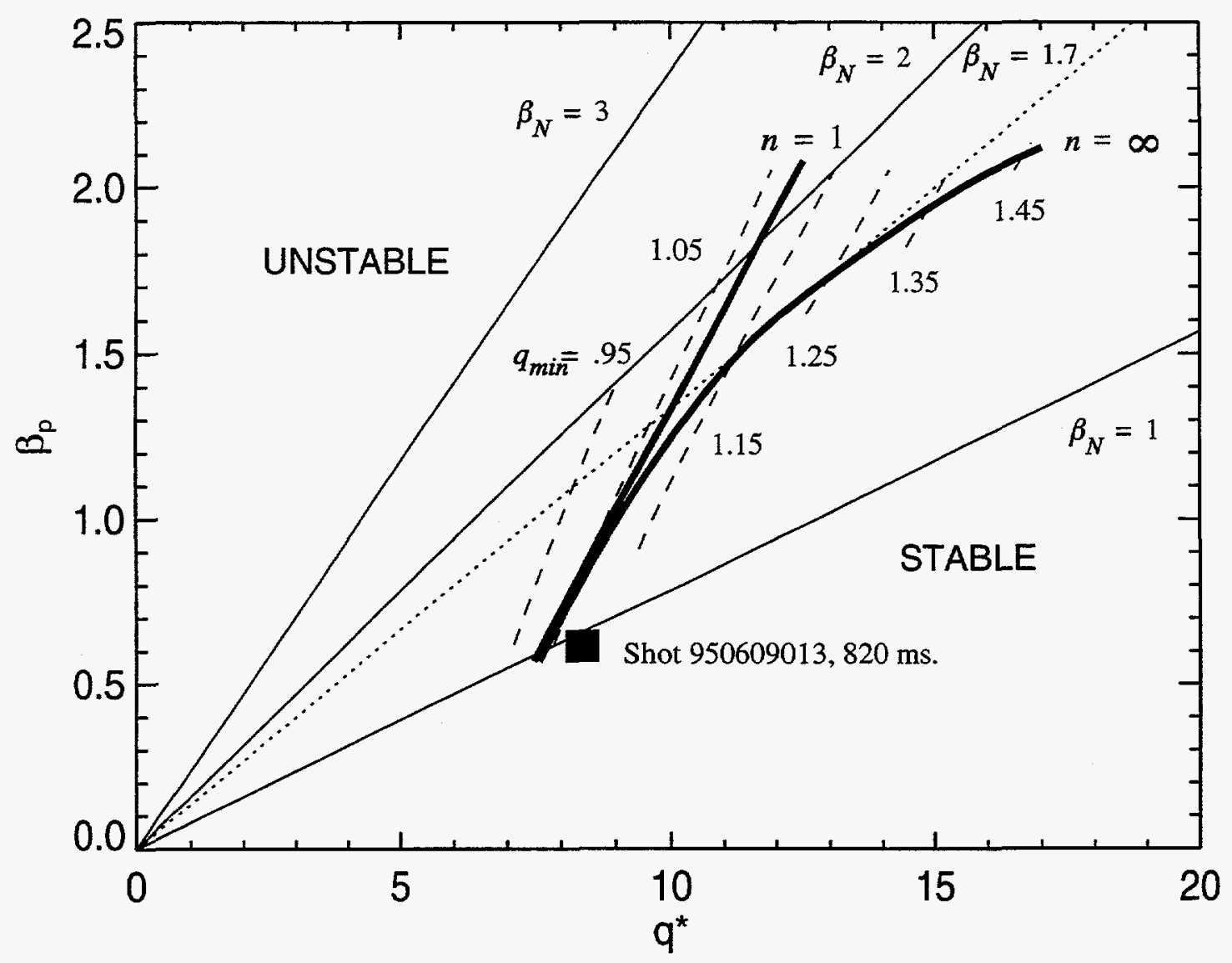

Figure 4.18: Ideal stability of shot 950609013 at $820 \mathrm{~ms}$ and discharges with similar equilibria. Equilibria that fall to the left or above the heavy lines are unstable to ideal modes.

the temperature in the outer part of the plasma increases, indicating that the mode is responsible for a degradation in the thermal confinement of the plasma core.

Paying close attention to the structure of the mode shown in the upper plot, one notices that as the temperature drops on one side of the axis, it rises on the other side. This antisymmetric behavior indicates that the mode has odd poloidal structure or odd $m$ number. This data combined with data from other diagnostics indicate that the mode has $n=1, m=1$ structure, consistent with the prediction of an internal 

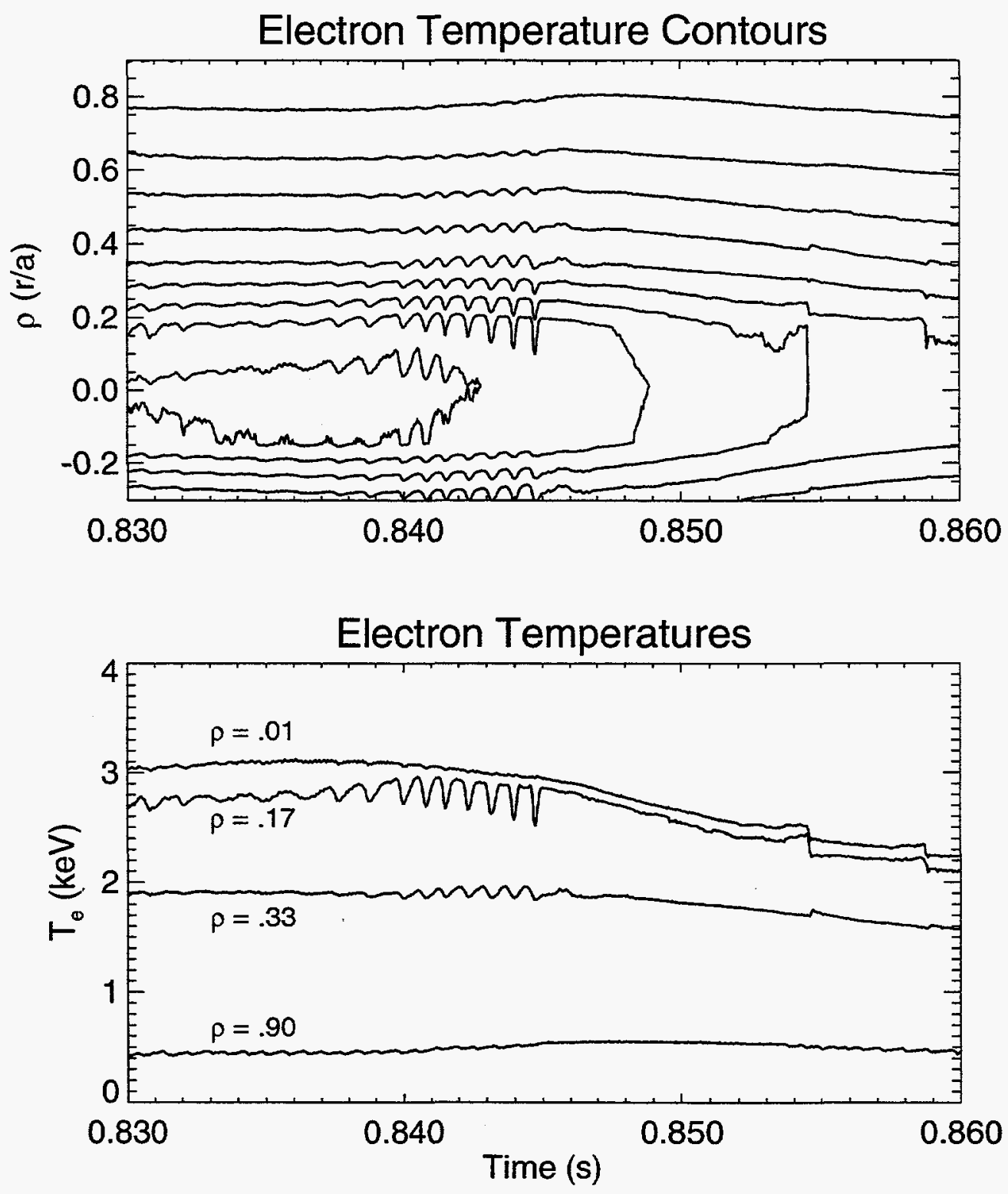

Figure 4.19: Shown above is a contour plot of electron temperature radial profile versus time from the grating polychromator (GPC) ECE diagnostic near the termination of enhanced confinement for shot 950609013. The actual temperature signals (lower plot) show clearly the loss of central enhanced confinement. 
kink. Furthermore, the peak of the mode amplitude is located at $\rho \approx .2$, near the $q_{\min }$ radius, as expected from the kink mode structure predicted by the stability analysis.

While this $n=1, m=1$ mode is present during the collapse of many of the high performance PEP modes and often is correlated with the loss of core enhanced confinement, the mode may not be the cause of the collapse but instead a consequence. Although difficult to tell from Fig. 4.18, the ideal stability analysis of these discharges would have the high- $n$ ballooning modes become unstable slightly before the $n=1$ mode, even for small values of $q_{\min }$ and $\beta_{p}$. The ballooning modes, because of their high spatial frequency and smaller extent would not be visible as coherent oscillations on the ordinary diagnostic signals. Thus, these modes may invisibly initiate the loss in enhanced confinement. Once the confinement degrades, the equilibrium profiles quickly change-as the bootstrap current is no longer driven-and other modes can be excited. Furthermore, the invisibility of these high- $n$ ballooning modes may explain those PEP mode discharges whose confinement collapses without the observation of coherent modes.

Of course, a caveat is warranted. Ideal MHD modes are not the only means a plasma will use to dissipate the high stored energy of these PEP mode discharges. Resistive modes may be important, as well as the simple resumption of whatever turbulent modes drive the anomalous transport in L-mode plasmas. Still, the nearness of these discharges to the marginal ideal stability limits demonstrates the plausibility that ideal MHD stability is important in these discharges. A few new core fluctuation diagnostics are being planned for installation on Alcator C-Mod. When these diagnostics become available, a return to this investigation may shed further light on this issue. 


\subsection{Relationship with other enhanced confinement modes}

\subsubsection{NCS/ERS mode}

Recently, much attention has been focused on enhanced core confinement discharges. Results from the DIII-D [56] and TFTR [57] tokamaks have demonstrated that a mode or modes of improved core confinement can by induced in discharges with reversed magnetic shear equilibria. Discovered independently, these modes are termed "Negative Central Shear" or NCS-mode (DIII-D) and "Enhanced Reverse Shear" or ERS-mode (TFTR). As we shall see, these modes are, in many ways, similar to PEP mode discharges.

To create NCS/ERS modes, a discharge is started and allowed to grow to its full size substantially before the full plasma current is obtained. The plasma is then centrally heated and fueled with neutral beam injection (NBI) to raise the plasma temperature and drop the central resistivity. This "freezes in" the current density at the core. The current is then ramped up to its full value, but because the resistivity is low in core of the plasma, the current cannot quickly diffuse inward. The resulting current density profile is hollow, giving the central region negative or reversed magnetic shear. After this "prelude" stage is accomplished and the reversed shear is established, large amounts of neutral beams are applied which heat and fuel the central plasma. When enough neutral beam power is applied, the discharge makes a transition to a mode of improved core confinement.

During the period of enhanced confinement of NCS/ERS discharges, the central core particle and thermal diffusivities are substantially reduced; in fact, the cores of these discharges are often described as particle and density integrators. Like the PEP mode discharges, this leads to large central pressures with steep pressure gradients in 
the inner half of the discharge. This increases the off-axis bootstrap current density, enhancing the shear reversal. Still, these modes are also transient. The large NBI powers typically drive these discharges to $\beta$ limit minor and major disruptions as current diffuses inward and $q_{\min }$ descends through rational numbers.

Analysis of the thermal transport of ERS modes reveals particle diffusivity near to the neoclassical value and ion thermal diffusivity substantially below neoclassical [57]. This apparent difficulty of observed thermal transport significantly below the irreducible minimum level may be ameliorated by correcting neoclassical theory for small gradient scale lengths, or allowing the presence of a significant inward thermal convection. Particle diffusivity is also substantially reduced - up to forty times less than L-mode discharges-approaching neoclassical values.

Another characteristic of NCS/ERS modes are large toroidal and poloidal rotational flows. In NCS discharges, the toroidal ion flow speed approaches $v_{\phi i} \sim 5 \times 10^{5}$ $\mathrm{m} / \mathrm{s}$ driven by the unbalanced tangential NBI. Poloidal rotations as high as $v_{\theta} \sim$ $4 \times 10^{4} \mathrm{~m} / \mathrm{s}$ have also been recorded [58], with the peak rate of rotation occurring near the steep pressure gradient of the transport barrier. Additionally, the gradient scale length of the poloidal rotation is small, similar to that of the pressure gradient scale length, indicating a large rotational shear, $d v / d r$.

Obviously, the similarities between NCS/ERS mode discharges and PEP modes are many. Common traits include: the existence of a sawtooth-free central region of reversed magnetic shear, significant reduction in particle and thermal transport to near ion neoclassical levels, and extremely peaked pressure profiles giving rise to significant amounts of bootstrap current. Not to be overlooked, another common trait is that both PEP and NCS/ERS modes are created by central fueling of the plasma. In the case of PEP modes, this fueling is accomplished by the injection of a pellet, while for the NCS/ERS modes, the fueling is provided by the high power neutral beams in a steady state manner. 


\subsubsection{Other modes}

Other modes of enhanced confinement have also been identified. For example, the high- $\beta_{p}$ modes of JT-60U [59] and DIII-D [60] both exhibit enhanced particle and energy confinement with extremely peaked pressure profiles. Like the NCS/ERS modes, they are characterized by significant toroidal and poloidal rotation. For DIIID high- $\beta_{p}$ discharges, maximum poloidal rotation velocities of $8 \times 10^{3} \mathrm{~m} / \mathrm{s}$ have been reported. As these modes are created without special startup treatments, they do not contain a region of reversed magnetic shear. However, as the pressure profile builds, significant bootstrap current develops - up to $80 \%$ of the total current in DIII-D high$\beta_{p}$ discharges -and the discharges develop an overall high $q$ profile with small, or possibly negative, magnetic shear. Thus analysis of these plasmas indicates that the central region of these discharges are in the second stability regime to ideal ballooning modes.

Another interesting example of enhanced core confinement is the $\mathrm{CH}$-mode discovered on PBX-M [61]. These modes appear to have all of the same characteristics of the ERS/NCS and PEP modes, except they are stimulated using a combination of NBI and off-axis Ion Bernstein Wave (IBW) ICRF heating. The IBW stimulates poloidal flow in a narrow region where the IBW are resonantly damped. By making this layer off-axis, a region of enhanced confinement is created within the resonant layer that leads to peaked pressure profiles and thus bootstrap current and reversed magnetic shear.

Clearly, these core enhanced confinement modes have many characteristics in com-

mon. It is therefore reasonable to assume that the same physical mechanism is at work to cause the suppression of the anomalous transport in the center of these discharges. In the next section, we will explore possible mechanisms for enhanced confinement in these discharges. 


\subsection{Theoretical Implications and Speculation}

While numerous theories have been advanced over the years to explain the reduction in transport observed during $\mathrm{P} / \mathrm{PEP}$ and other enhanced confinement modes, recently a framework to include may of these concepts has emerged. First of all, we suppose that such a reduction in transport is achieved by suppression of turbulent modes that are responsible for anomalous transport. This supposition is bolstered, at least for the case of NCS/ERS modes, by experimental evidence showing large reductions in fluctuation levels concurrent with the improvement in confinement. The notion that the suppression of these turbulent modes is achieved by "shear flow decorrelation" is generally regarded as valid [62-66]. Schematically, this means that the small turbulent eddies that drive transport are torn apart by relative motion of the plasma across the eddies. Mathematically, the condition for suppression of turbulence is

$$
\gamma_{\text {mode }}<\omega_{s}
$$

ie. the growth rate of the turbulent mode, $\gamma_{\text {mode }}$, must be less than the "shearing rate", $\omega_{s}$. While the exact form of $\omega_{s}$ is a function of the spatial structure of a particular mode, in general for flute-like modes-modes with high poloidal mode numbers and large toroidal extent-for toroidal geometry $[64,66]$

$$
\omega_{s} \propto\left|\frac{\partial}{\partial r} \frac{E_{r}}{R B_{\theta}}\right|
$$

where $E_{r}$ is the radial electric field and $B_{\theta}$ is the poloidal magnetic field. Assuming radial force balance, the two-fluid momentum equations give relations for $E_{r}$ :

$$
E_{r}=\frac{\nabla p_{j}}{e_{j} n_{j} Z_{j}}-v_{\theta j} B_{\phi}+v_{\phi j} B_{\theta}
$$


where $v_{\theta j}$ and $v_{\phi j}$ are poloidal and toroidal flow velocities of each species, respectively. Thus, the relative importance of poloidal flow shear is revealed. We will return to the subject of poloidal rotation shortly.

\subsection{1 $\eta_{i}$ Driven Modes}

Of course, the left hand side of Eq. (4.14) is equally important. To calculate $\gamma_{\text {mode }}$, however, we must assume to know the nature of the modes responsible for anomalous transport. Trapped particle drift modes, driven by ion temperature gradients, have long been regarded as a likely candidate for anomalous transport turbulence [65, 6770]. A critical parameter for these modes is the ratio of the ion temperature scale length to density scale length, $\eta_{i}$.

$$
\eta_{i}=\frac{\partial \ln T_{i}}{\partial \ln n_{i}}
$$

Thus, these modes are often referred to as $\eta_{i}$ modes. Numerical simulation of the ( $\eta_{i}$ driven) trapped electron mode (TEM) for TFTR discharge conditions [71] reveals a first-order growth rate dependence for $\eta_{i}$ modes:

$$
\gamma_{T E M} \sim G_{1}\left(\eta_{i}, \ldots\right)+G_{2} \hat{s}-G_{3} \alpha+\ldots
$$

where $G_{1}$ is the temperature gradient driving term and $G_{2}$ and $G_{3}$ are non-negative. The magnetic shear $\hat{s}$ is given by $\hat{s} \equiv d \ln q / d \ln r$, while $\alpha$, the normalized pressure gradient, is given by $\alpha \equiv-q^{2} R_{0} d \beta / d r$ where $\beta \equiv 2 \mu_{0} p / B^{2}$.

\subsubsection{Mechanisms for reduced transport}

While early attempts to explain $\mathrm{P} / \mathrm{PEP}$ modes focused on finding a fixed critical value for $\eta_{i}$ over which anomalous transport exists, the larger framework of turbulence 
decorrelation offers further insights. Clearly, a sufficient reduction in $\eta_{i}$ is capable of reducing the growth rates of these modes. Such a condition may exist shortly after the injection of the pellet when the temperature profile is nearly flat and the density profile is extremely peaked. However, as the PEP mode evolves and the ion temperature profile becomes very peaked, values for $\eta_{i}$ return to near L-mode levels. ${ }^{9}$

\section{Reversed Shear}

The positive dependence of the growth rate on the magnetic shear, indicated by positive $G_{2}$ in Eq. (4.18), is a result of favorable geodesic curvature as discussed in $\S 2.5$. Thus, we see that negative magnetic shear has a damping effect on these turbulent modes. In this larger theoretical framework, however, it is easier to understand why reversed shear, i.e. $\hat{s}<0$, is not a sufficient and potentially not a necessary condition for enhanced confinement. However, small magnetic shear is necessary for stability to the high- $n$ ideal ballooning modes for these high $\beta$ discharges. Furthermore, J. Drake has suggested that, based on numerical modelling studies of TFTR discharges, the improvement in confinement in ERS modes may be explained by stabilization of resistive ballooning modes through reversal of the local magnetic shear [73].

\section{Pressure Gradient}

The high pressure gradient of core enhanced confinement discharges plays a triple role. First, as previously discussed, it produces bootstrap current which in turn reduces or inverts the magnetic shear in the center of these discharges. Furthermore, as evident by simple inspection of Eq. (4.18), a strong pressure gradient, $\alpha$, reduces the growth rate of the turbulent modes. Large $\alpha$ is a result not only of the high value of $\beta$ on axis, but is further increased by the radial compression of flux on the outside of

\footnotetext{
${ }^{9}$ The dependence of the growth rate on $\eta_{i}$ is complex. A recent theory suggests that these modes have a second region of stability for sufficiently high values of $\eta_{i}[72]$.
} 
the plasma, the Shafranov shift. The significant Shafranov shift in these discharges is also a result of the high central $\beta$ as well as the potentially high values of $q_{0}$ in these reversed shear equilibria.

Finally, as evident in the force balance equation (4.16), the existence of a peaked pressure profile can also influence the radial electric field, thereby increasing the shearing rate, $\omega_{s}$, and suppressing turbulence. For shot 950609013 , the contribution to the radial electric field from the ion pressure gradient is as high as $40 \mathrm{kV} / \mathrm{m}$ at $\rho=0.2$, implying an ion diamagnetic poloidal rotation speed of $\approx 8 \times 10^{3} \mathrm{~m} / \mathrm{s}$.

\section{Poloidal Rotation}

Large poloidal flows have been measured in nearly all enhanced confinement regimes: including NCS/ERS mode, high- $\beta_{p}$ discharges, CH- and VH-modes, as well as the much studied $\mathrm{H}$-mode. That no poloidal flow, to within our experimental resolution, has been measured for PEP modes, is a surprising and somewhat troubling resultalthough the existence of a moderately high poloidal rotation of $\sim 1 \times 10^{4} \mathrm{~m} / \mathrm{s}$ would not be inconsistent with present experimental results.

The source of poloidal rotation in enhanced confinement modes is worth some investigation. In some cases, the source of poloidal rotation is well known, such as unbalanced NBI or IBW injection. In others, the source of rotation is not well understood, especially considering that neoclassical transport predicts that poloidal rotation is strongly damped.

One possible mechanism for driving poloidal rotation is called "Stringer Spinup" $[74,75]$. While the physical mechanism for the spin-up is subtle and goes beyond the scope of this thesis, ${ }^{10}$ a few facts may elucidate its importance for PEP modes. Stringer spin-up is an instability in a toroidal plasma driven by the outward gravita-

\footnotetext{
${ }^{10}$ The curious reader is referred to the derivation by Hassam and Drake [75].
} 
tional (centripetal) force ${ }^{11}$

$$
g=\frac{c_{s}^{2}}{R}
$$

where the sound speed given by $c_{s}=\sqrt{\gamma T_{e} / m_{i}}$. Ordinarily, poloidal rotation is damped, but when a flux surface density asymmetry arises, due to asymmetric particle sources or transport, the growth rate for this instability can become positive. Hassam has suggested [76] that sufficient flux surface density asymmetry might also arise in PEP modes due to the in-out asymmetry of the large Shafranov shifts involved, coupled with the large density gradients. A positive growth rate leads to an overall poloidal spin-up of the plasma saturating at the poloidal sound speed,

$$
c_{p s}=\left(\frac{B_{p}}{B}\right) c_{s}
$$

Thus for PEP mode discharges, the theory of Stringer spin-up predicts a poloidal rotation of $\sim 3.5 \times 10^{4} \mathrm{~m} / \mathrm{s}$. However, such rotation speeds are inconsistent with the data gathered from the Alcator C-Mod HIREX diagnostic.

\subsubsection{Feedback and Causality}

Thus far, we have noted numerous mechanisms that may be acting, either separately or together, to produce the region of enhanced confinement in PEP mode discharges. One may further note that some of these parameters, such as the pressure gradient, are increased as a result of the enhanced confinement. This implies that a system of positive feedback exists. From the arguments put forth in the previous section, a few

\footnotetext{
${ }^{11}$ This same force also drives high-n ballooning modes.
} 
such feedback mechanisms are shown schematically below.

$$
\uparrow \nabla p \Rightarrow\left\{\begin{aligned}
\uparrow j_{B S} \Rightarrow \downarrow \hat{s} & \Rightarrow \downarrow \gamma_{\eta_{i}} \Rightarrow \downarrow \chi_{i} \\
\uparrow \alpha & \Rightarrow \downarrow \gamma_{\eta_{i}} \Rightarrow \downarrow \chi_{i} \\
& \uparrow E_{r} \Rightarrow \uparrow \omega_{s} \Rightarrow \downarrow \chi_{i}
\end{aligned}\right\} \Rightarrow \uparrow p
$$

For example, an increase in pressure gradient, $\nabla p$, leads to higher bootstrap current, $j_{B S}$, which lowers the magnetic shear, $\hat{s}$, and thus decreases the growth rate for $\eta_{i}$ modes, $\gamma_{\eta_{i}}$. This implies a reduced level of turbulence and a concommitant reduction in thermal transport parameters, $\chi_{i}$, which leads to high pressure in enhanced confinement region and thus a higher pressure gradient, $\nabla p$. A similar argument applies for the pressure gradient affecting $\alpha$ and $E_{r}$. In short, a pressure gradient drives a higher pressure gradient. This existence of positive feedback in the system implies that a bifurcation between L-mode and enhanced discharges should exist. Conversely, the observation of sharp temporal and spatial bifurcation between the L-mode and PEP mode confinement suggests that a mechanism positive feedback of must be employed to explain the improvement in confinement.

Finally, as any feedback mechanism is inherently cyclical, determining which of the many characteristics of enhanced core confinement modes is responsible for the improvement in confinement is difficult indeed. ${ }^{12}$ However, we can make a few statements about the causality of these modes. We know that PEP modes are created by the injection of a pellet. This produces an initial density profile that is very peaked after which the core density and density gradients gradually drop until the mode collapses. Furthermore, other enhanced core confinement modes are created with high-power, core-deposited NBI-another form of core particle fueling. Thus, we may hypothesize that core enhanced confinement is caused by central particle fueling. Suggestions for future experiments based on this hypothesis will be given in $§ 5.2$.

\footnotetext{
${ }^{12} \mathrm{~A}$ bit like trying to answer the "chicken and the egg" question.
} 


\section{Chapter 5}

\section{Conclusion}

\subsection{Summary}

This thesis has discussed experiments involving lithium pellet injection into the Alcator C-Mod tokamak with a particular focus on the improvement of confinement observed after pellet injection. We began with a brief review of magnetic confinement of plasmas, introducing the tokamak, MHD equilibrium and stability, and transport. A link between equilibrium and anomalous transport was presented, highlighting the importance of the $q$ profile.

\section{Lithium Pellet Injection ' $q$ ' profile diagnostic}

Measurement of current density profiles is accomplished on Alcator C-Mod using high speed lithium pellets as a means of measuring the local magnetic field tilt angle within the plasma. Two different techniques have been applied. The first, known as the 'Cigar Detector' relies on the observation that the pellet ablation emission cloud is elongated and cigar-like in shape. Because ions are confined to move along field lines, imaging the $\mathrm{Li}^{+}$ion emission gives the direction of the magnetic field. This imag- 
ing is accomplished using a fast-gated CCD camera. The second technique involves polarimetry of the Zeeman split emission from lithiun ions within the pellet ablation cloud. To complete the diagnostic, the trajectory of the pellet is determined using a 3-D stereoscopic tracking system. The data from the internal magnetic field tilt angle measurement, the pellet tracking system, and external magnetic measurements, are used to numerically solve the Grad-Shafranov equation for the magnetic equilibrium of the plasma, from which the current density and $q$ profiles are determined.

Results from the Cigar Detector are quite encouraging. Estimates of the error in measured tilt angle are as low as $0.1^{\circ}$. This technique was used to determine the $q$ profile during PEP mode discharges demonstrating that within the experimental error a region of weak to moderate reversed shear exists near the plasma core. The main drawback of this technique, as implemented, is the requirement of many repeatable discharges to obtain a single tilt angle profile. This requirement could be eliminated if a more advanced imaging system were implemented.

\section{Pellet Enhanced Performance}

A transient mode of enhanced core confinement has been observed following the injection of deeply penetrating lithium pellets into Alcator C-Mod discharges. These pellet enhanced performance (PEP) modes, are characterized by a steep density, ion temperature and total pressure gradients. The pressure gradient, $\ell_{p}$, is as small as $(a / 10)$ in the inner third of the plasma, indicating the presence of an internal transport barrier. Inside this barrier, particle and energy diffusivities are greatly reduced, with ion thermal diffusivity dropping to near neoclassical values. Meanwhile, the global energy confinement time shows a 30\% improvement over ITER89-P L-mode scaling. The addition of ICRF auxiliary heating shortly after the pellet injection leads to high fusion reactivity with neutron rates enhanced by a factor of 10 over L-mode discharges with similar input powers. 
The current density profile, as determined by ablation cloud tilt angle measurements of a second pellet injected during the period of good confinement, indicates that a region of reversed magnetic shear exists at the plasma core. This current density profile is consistent with TRANSP calculations for the bootstrap current created by the pressure gradient. Results from ideal MHD stability analysis of these discharges indicates that these plasmas are near both the $n=\infty$ and the $n=1$ marginal stability limits.

\section{$5.2 \quad$ Further Investigations}

Like most experiments, those presented in this document leave some questions unanswered and beg others. In this section, an outline of possible future exploration is suggested.

In some cases, new or improved diagnostic ability would lend further understanding of PEP mode discharges. Planned upgrades to the Alcator C-Mod diagnostic set include a tangential interferometer, an X-mode reflectometer and upgrades to the Thomson scattering diagnostic to provide more channels. Combined with the measurements from the TCI (the existing interferometer diagnostic), these upgrades will provide accurate electron density profile information with high spatial and temporal resolution. This data will permit quantitative study of the particle transport and allow the evolution of transport barrier position to be carefully studied. Coupled with data from the existing visible bremsstrahlung diagnostic (Z-meter), better $Z_{\text {eff }}$ profiles will also become available for impurity transport analysis and more accurate $q$ profile modelling by TRANSP.

The planned installation of a diagnostic neutral beam (DNB) will also open many avenues for investigation. The associate charge-exchange and recombination spectrometer systems (CHERS) will provide high resolution measurement of the ion tem- 
perature and velocity. The improved $T_{i}$ profile data expected from this system will greatly aid the accuracy of the thermal transport analysis of these discharges. The additionally capability of measuring $v_{i \phi}$ and $v_{i \theta}$ will allow determination of the $E_{r}$ profile, and thus a test of the shear flow decorrelation theory discussed in $\S 4.6$.

The beam emission spectrometer system (BES) will measure fluctuations of electron density, $\tilde{n}_{e}$, in the core of the discharge. The planned improvement to the reflectometers should also provide mearurements of $\tilde{n}_{e}$ during PEP modes. We have speculated that confinement enhancement in PEP mode discharges is due to suppression of turbulent modes; fluctuation spectra from the BES diagnostic will test this notion.

We have shown that a region of reversed magnetic shear exists during PEP discharges. However, the causal relationship of reversed shear and enhanced confinement has not been established. Thus, further measurements of $q$ profiles during PEP modes are desirable. A motional stark effect (MSE) $q$ profile diagnostic has been planned for as a further upgrade to the DNB systems; however, the beam may not be capable of giving the necessary spatial resolution near the magnetic axis. Given the promising results of the LPI cigar detector diagnostic, the implementation of a high-speed digital camera, capable of taking several images of a pellet ablation cloud, is also suggested.

Several possible experiments may also yield data useful for forming a better understanding of core enhanced confinement modes. As previously mentioned, the core enhanced confinement modes, including PEP, high- $\beta_{p}$ and $\mathrm{CH}$ - modes, share the trait of being created using core particle fueling. One set of experiments designed to test if central fueling is a necessary pre-condition for enhanced confinement would include attempting to create the NCS/ERS mode on Alcator C-Mod without pellet or neutral beam injection and just large amounts of ICRF auxiliary heating. Alternatively, on a machine such as DIII-D where the power threshold for transition to NCS mode is well 
characterized, one may attempt to obtain transition to enhanced confinement with less neutral beam power supplemented with other auxiliary heating such as ICRF or $\mathrm{ECH}$. If it turns out that central particle fueling is one of the necessary conditions to access these enhanced core confinement modes, then extrapolation to future devices (such as ITER) may be difficult. 


\section{Bibliography}

[1] F. F. Chen. Introduction to Plasma Physics and Controlled Fusion, volume 1. Plenum Press, $2^{\text {nd }}$ edition, 1984.

[2] D. R. Nicholson. Introduction to Plasma Theory. John Wiley \& Sons, 1983.

[3] R. D. Hazeltine and J. D. Meiss. Plasma Confinement. Addison Wesley, 1992.

[4] E. S. Marmar. The Alcator C-Mod diagnostic complement. Rev. Sci. Instrum., 63(10):4695, 1992.

[5] I. H. Hutchinson. Principles of plasma diagnostics. Cambridge University Press, 1987.

[6] J. P. Freidberg. Ideal Magnetohydrodynamics. Plenum Press, 1987.

[7] S. von Goeler, W. Stodiek, and N. Sauthoff. Studies of internal disruptions and $m=1$ oscillations in tokamak discharges with soft-x-ray techniques. Phys. Rev. Lett., 33(20):1201, 1974.

[8] F. L. Hinton and R. D. Hazeltine. Theory of plasma transport in toroidal confinement systems. Rev. Mod. Phys., 48(2):239, 1976.

[9] A. A. Ware. Pinch effect for trapped particles in a tokamak. Phys. Rev. Lett., 25:15, 1970.

[10] S. I. Braginskii. Transport processes in a plasma. In M. A. Leontovich, editor, Reviews of Plasma Physics, pages 205-311. Consultants Bureau, New York, 1965.

[11] L. L. Lao, H. St. John, R. D. Stambaugh, A. G. Kellman, and W. Pfeiffer. Reconstruction of current profile parameters and plasma shapes in tokamaks. Nucl. Fusion, 25(11):1611, 1985.

[12] L. L. Lao, J. R. Ferron, R. J. Groebner, W. Howl, H. St. John, E. J. Strait, and T. S. Taylor. Equilibrium analysis of current profiles in tokamaks. Nucl. Fusion, 30(6):1035, 1990. 
[13] L. L. Lao et al. Nucl. Fusion, 25:1421, 1985.

[14] F. Alladio and P. Micozzi. Experimental plasma equilibrium reconstruction from kinetic and magnetic measurements in the FTU tokamak. Nucl. Fusion, 35(3):305, 1995.

[15] J. P. Freidberg, M. Graf, A. Niemszewski, S. Schultz, and A. Shagii. Why $\beta_{p}$ and $\ell_{i}$ cannot be separately measured in a near circular tokamak. Plasma Phys. Control. Fusion, 35:1641, 1993.

[16] R. S. Granetz et al. Killer pellet experiments on the Alcator C-Mod tokamak. Bull. Am. Phys. Soc., 40, Nov. 1995.

[17] P. B. Parks, R. J. Turnbull, and C. A. Foster. A model for the ablation rate of a solid hydrogen pellet in a plasma. Nucl. Fusion, 17(3):539, 1977.

[18] S. L. Milora, W. A. Houlberg, L. L. Lengyel, and V. Mertens. Pellet fuelling. Nucl. Fusion, 35(6):657, 1995.

[19] P. B. Parks. Electric field and current distribution near the ablation cloud of a pellet injected into a tokamak. Nucl. Fusion, 32(12):2137, 1992.

[20] J. A. Snipes, E. S. Marmar, J. L. Terry, et al. Wall conditioning with impurity pellet injection on TFTR. J. Nucl. Mater., 196-198:686-691, 1992.

[21] G. L. Jackson. private communication.

[22] S. L. Milora, S. K. Combs, and C. R. Foust. Fast-opening magnetic valve for high-pressure gas injection and applications to hydrogen pellet fueling systems (plasma fusion confinement). Rev. Sci. Instrum., 57(9):2356, 1986.

[23] P. Horowitz and W. Hill. The Art of Electronics. Cambridge University Press, $2^{\text {nd }}$ edition, 1993.

[24] S. M. Egorov, B. V. Kuteev, I. V. Miroshnikov, A. A. Mikhailenko, V. Yu. Sergeev, S. N. Ushakov, et al. Current density profile and electron beam localization measurements using carbon pellets on T-10. Nucl. Fusion, 32(11):2025, 1992.

[25] B. V. Kuteev. Pellet ablation in the Large Helical Device. Technical Report NIFS-260, National Institute for Fusion Science, Nov. 1993.

[26] TFR Group. Deuterium pellet injection into plasmas of the Fontenay-aux-Roses tokamak TFR: Photographic and spectroscopic measurements of the ablation zone. Europhys. Lett., 2(4):267, 1986. 
[27] S. M. Egorov, B. V. Kuteev, I. V. Miroshnikov, and V. Yu. Sergeev. Observation of a magnetic field line in the T-10 tokamak. JETP Lett., 46(4):180, 1987.

[28] R. D. Durst, P. E. Phillips, and W. Rowan. q-profile measurement in tokamaks using fueling pellets. Rev. Sci. Instrum., 59(8):1623, 1988.

[29] J. L. Terry, E. S. Marmar, J. A. Snipes, D. Garnier, and V. Yu. Sergeev. Imaging of lithium pellet ablation trails and measurement of $q$ profiles in TFTR. Rev. Sci. Instrum., 63(10):5191, 1992.

[30] I. V. Misoshnikov. New instrument for measuring magnetic-field tilt profile and pellet clouds emission structure. Rev. Sci. Instrum., 66(1):389, Jan. 1995.

[31] J. A. Urbahn. The Alcator C-Mod Deuterium Pellet Injector. PhD thesis, Massachusetts Institute of Technology, 1994.

[32] E. S. Marmar, J. L. Terry, B. Lipschultz, and J. E. Rice. Measurement of the current density profile in the Alcator C tokamak using lithium pellets. Rev. Sci. Instrum., 60(12):3739, 1989.

[33] V. Rozhansky, I. Veselova, and S. Voskoboynikov. Evolution and stratification of plasma cloud surrounding a pellet. Plasma Phys. Control. Fusion, 37:399, 1995.

[34] E. S. Marmar, J. Snipes, et al. Tftr double pellet.

[35] K. L. Bell, H. B. Gilbody, J. G. Hughes, A. E. Kingston, and F. J. Smith. Recommended data on the electron impact ionization of light atoms and ions. $J$. Phys. Chem. Ref. Data, 12(4):891, 1983.

[36] C. K. Phillips, J. Hosea, E. Marmar, M. W. Phillips, J. Snipes, J. Stevens, J. Terry, J. R. Wilson, et al. Ion cyclotron range of frequencies stabilization of sawteeth on tokamak fusion test reactor. Phys. Fluids B, 4(7):2155, 1992.

[37] L. Spitzer, Jr., D. J. Grove, W. E. Tonks, and W. R. Westendorp. Problems of the stellarator as a useful power source. Technical Report NYO 6047, USAEC, 1954 .

[38] M. Greenwald et al. Phys. Rev. Lett., 53:352, 1984.

[39] M. Hugon, B. P. van Milligen, P. Smeulders, et al. Shear reversal and MHD activity during pellet enhanced performance pulses in JET. Nucl. Fusion, 32(1), 1992.

[40] P. Smeulders et al. Survey of pellet enhanced performance in JET discharges. Nucl. Fusion, 35(2), 1995. 
[41] M. Greenwald and the Alcator Group. Confinement of Ohmic and ICRF heated plasmas in Alcator C-Mod. Plasma Phys. Control. Fusion, 1995.

[42] P. N. Yushmanov, T. Takizuka, K. S. Riedel, O. J. W. F. Kardaun, J. G. Cordey, S. M. Kaye, and D. E. Post. Scalings for tokamak energy confinement. Nucl. Fusion, 30(10):1999, 1990.

[43] M. J. May, A. P. Zwicker, M. Finkenthal, H. W. Moos, and J. L. Terry. MLM based monochromator for molybdenum impurity monitoring on the Alcator CMod tokamak in the 30-130 Ang range. Rev. Sci. Instrum., 63(10):5176, 1992.

[44] P. H. Rutherford. Phys. Fluids, 17:1782, 1974.

[45] R. D. Petrasso, J. E. Hopf, K. W. Wenzel, D. J. Sigmar, J. L. Terry, and M. Greenwald. Observations of centrally peaked impurity profiles following pellet injection in the alcator-c tokamak. Rev. Sci. Instrum., 57(8):2195, 1986.

[46] R. J. Hawryluk. An empirical approach to tokamak transport. In B. Coppi et al., editors, Physics of Plasmas Close to Thermonuclear Conditions, volume 1, page 19. CEC, Brussels, 1980.

[47] J. Schachter et al. Local transport analysis for Alcator C-Mod. Bull. Am. Phys. Soc., 39:1666, Nov. 1994.

[48] G. W. Hammett. Fast Ion Studies of Ion Cyclotron Heating in the PLT Tokamak. $\mathrm{PhD}$ thesis, Princeton, 1986.

[49] M. Evrard, J. Ongena, and D. van Eester. Improved dielectric tensor in the ICRF module of TRANSP. In Proceedings of the Tenth Topical Conference on Radio-Frequency Power in Plasmas. AIP, 1995.

[50] C. S. Chang and F. L. Hinton. Effect of finite aspect ratio on the neoclassical ion thermal conductivity in the banana regime. Phys. Fluids, 25(9):1493, 1982.

[51] K. C. Shaing and R. D. Hazeltine. Effects of orbit squeezing on ion transport in the banana regime in tokamaks. Phys. Fluids B, 4(8):2547, 1992.

[52] S. P. Hirshman, U. Schwenn, and J. Nuhrenberg. Improved radial differencing for three-dimensional magnetohydrodynamic equilibrium calculations. J. Comp. Phys., 87(2):396, 1990.

[53] M. C. Zarnstorff, K. McGuire, M. G. Bell, B. Grek, D. Johnson, D. McCune, H. Park, A. Ramsey, and G. Taylor. Parallel electric resistivity in the tftr tokamak. Phys. Fluids B, 2(8):1852, 1990. 
[54] M. V. Medvedev, L. Villard, L. M. Degtyarev, A. Martynov, R. Gruber, and F. Troyon. In Proceedings of the $20^{\text {th }}$ EPS Conf. on Cont. Fus. and Pl Phys., volume pt IV, page 1279, Lisbon, 1993.

[55] M. J. Gerver, J. Kesner, and J. J. Ramos. Access to the second stability region in a high-shear, low-aspect-ratio tokamak. Phys. Fluids, 31(9), 1988.

[56] E. J. Strait, L. L. Lao, M. E. Mauel, B. W. Rice, T. S. Taylor, et al. Enhanced confinement and stability in DIII-D discharges with reversed magnetic shear. Phys. Rev. Lett., 75(24):4421, 1995.

[57] F. M. Levinton, M. C. Zarnstorff, et al. Improved confinement with reversed magnetic shear in TFTR. Phys. Rev. Lett., 75(24):4417, 1995.

[58] K. Burrell et al. Ninth Transport Task Force Workshop, Philadelphia, Pennsylvania, March 12-16, 1996.

[59] T. Nishitani, S. Ishida, M. Kikuchi, M. Azumi, M. Yamagiwa, T. Fugita, Y. Kamada, Y. Kawano, Y. Koide, T. Hatae, M. Mori, and S. Tsuji. Attainment of high fusion reactivity under high bootstrap current fraction in JT-60U. Nucl. Fusion, 34(8):1069, 1994.

[60] B. W. Stallard, C. M. Greenfield, P. A. Polizer, and H. E. St. John. Transport in high $\beta_{p}$ discharges in DIII-D. Bull. Am. Phys. Soc., 40(11), Nov. 1994.

[61] PBX-M Group. Lower hybrid current drive and ion Bernstein wave heating experiments on PBX-M. Fusion Eng. Des., 26:83, Jan. 1995.

[62] H. Biglari, P. Diamond, and P. Terry. Influence of sheared poloidal rotation on edge turbulence. Phys. Fluids B, 2(1):1, 1990.

[63] G. M. Staebler, F. L. Hinton, J. C. Wiley, R. R. Dominguez, C. M. Greenfield, P. Gohil, T. K. Kurki-Suonio, and T. H. Osborne. High and very high modes from energy, particle, and momentum transport. Phys. Plasmas, 1(4):909, 1994.

[64] T. S. Hahm. Rotation shear induced fluctuation decorrelation in a toroidal plasma. Phys. Plasmas, 1(9):2940, 1994.

[65] M. Kotschenreuther, W. Dorland, M. A. Beer, and G. W. Hammett. Quantitative predictions of tokamak energy confinement from first-principles simulations with kinetic effects. Phys. Plasmas, 2(6):2381, 1995.

[66] T. S. Hahm and K. H. Burrell. Flow shear induced fluctuation suppression in finite aspect ratio shaped tokamak plasma. Phys. Plasmas, 2(5):1648, 1995. 
[67] M. Porkolab and G. Kino. Phys. Rev. Lett., 15:752, 1965.

[68] B. Coppi, M. N. Rosenbluth, and R. Z. Sagdeev. Instabilities due to temperature gradients in complex magnetic field configurations. Phys. Fluids, 10(3):582, 1967.

[69] M. Porkolab. Plasma instabilities due to ion temperature gradients. Nucl. Fusion, 8:29, 1968.

[70] B. Coppi, S. Migliuolo, and Y.-K. Pu. Candidate mode for electron thermal energy transport in multi-keV plasmas. Phys. Fluids B, 2(10):2322, 1990.

[71] M. Beer et al. Ninth Transport Task Force Workshop, Philadelphia, Pennsylvania, March 12-16, 1996.

[72] H. Song and A. K. Sen. Second stable regime of mixed slab-toroidal ITG mode with anisotropic $\eta_{i}$. International Sherwood Fusion Theory Conference, Philadelphia, Pennsylvania, March 18-20 1996.

[73] J. F. Drake, Y. T. Lau, P. N. Guzdar, A. B. Hassam, S. V. Novakovski, B. Rogers, and A. Zeiler. Local negative shear and the formation of transport barriers. Phys. Rev. Lett., 1996. submitted to.

[74] T. E. Stringer. Phys. Rev. Lett., 22:1770, 1969.

[75] A. B. Hassam and J. F. Drake. Spontaneous poloidal spin-up of tokamak plasmas: Reduced equations, physical mechanism, and sonic regimes. Phys. Fluids B, 5(11):4022, Nov. 1993.

[76] A. B. Hassam. Private communication, 1996. 